\title{
Fluctuations and Oscillatory Instabilities of Intracellular Fiber Networks
}

\author{
Dissertation \\ for the award of the degree \\ Doctor rerum naturalium \\ of the Georg-August-Universität Göttingen, \\ within the doctoral program \\ Physics of Biological and Complex Systems \\ of the Georg-August University School of Science (GAUSS)
}

\author{
submitted by \\ Jose Negrete Jr. \\ from \\ El Paso, Texas, United States of America
}

Göttingen, 2014 
Advisor:

Prof. Dr. Eberhard Bodenschatz

Laboratory for Fluid Dynamics, Pattern Formation and Biocomplexity - Max Planck Institute for Dynamics and Self-Organization / Laboratory of Atomic and Solid State Physics and Sibley School of Mechanical and Aerospace Engineering - Cornell University / Institute for Nonlinear Dynamics - Georg-August-Universität Göttingen

Thesis Committee:

Prof. Dr. Ulrich Parlitz

Biomedical Physics Group - Max Planck Institute for Dynamics and Self-Organization / Third Institute of Physics - Georg-August-Universität Göttingen

Prof. Dr. Sarah Köster

Institute for X-ray Physics - Georg-August-Universität Göttingen

Members of Examination Board:

Prof. Dr. Jörg Großhans

Institute of Developmental Biochemistry - Universitätsmedizin Göttingen

Dr. Andreas Neef

Department of Nonlinear Dynamics - Max Planck Institute for Dynamics and Self-Organization / Bernstein Center for Computational Neuroscience Göttingen

Dr. Reiner Kree Institute for Theoretical Physics - Georg-August-Universität Göttingen

Date of oral examination: December 3rd, 2014 
...there are also unknown unknowns - the ones we don't know we don't know. And if one looks throughout the history of our country and other free countries, it is the latter category that tend to be the difficult ones. -Donald Rumsfeld, 13th and 21st United States Secretary of Defense

...this little girl hardly paid attention, and in this drawing lesson she did. The teacher was fascinated and she went over to her and she said, "What are you drawing?" and the girl said, "I am drawing a picture of God" And the teacher said, "But nobody knows what God looks like." And the girl said, "They will in a minute!"

-Sir Ken Robinson, Educationalist 


\section{Nomenclature}

\begin{tabular}{|c|c|}
\hline+ & $\begin{array}{l}\text { If we have protein } \mathrm{A} \text { and protein } \mathrm{B} \text {, then } \mathrm{A}+\mathrm{B} \text { means } \\
\mathrm{A} \text { co-expressed with } \mathrm{B}\end{array}$ \\
\hline$-G F P$ or $-m R F P$ & $\begin{array}{l}\text { If we have protein A then A-GFP or A-mRFP, will } \\
\text { stand for protein A fused with green fluorescent pro- } \\
\text { tein or with monomeric red fluorescent protein. }\end{array}$ \\
\hline$\Delta$ & $\begin{array}{l}\text { Gene deleted, for example: if a gene produces protein } \\
A, \text { then } A \Delta \text { means that such gene was deleted }\end{array}$ \\
\hline$A b p$ & Actin binding protein \\
\hline$A D F$ & Actin depolymerization factor \\
\hline Aip 1 & Actin interacting protein 1 \\
\hline$A X-2$ & Dictyostelium discoideum axenic cell line 2 \\
\hline$B C M C M-c A M P$ & $\begin{array}{l}6,7 \text { - Bis(carboxymethoxy)coumarin- 4- yl)methyl-adenosine- } \\
3 \text { ', 5'- cyclic monophosphate }\end{array}$ \\
\hline$c A M P$ & 3'-5'-cyclic adenosine monophosphate \\
\hline$C A R$ & cAMP receptors \\
\hline Crn & Coronin 1A \\
\hline$F-$ Actin & Filamentous Actin \\
\hline$G A P$ & GTP-ase activating protein \\
\hline
\end{tabular}


$G D P$

$G E F$

$H L-5$

Lat A

PI3K

PIP2

PIP3

PTEN

$R B D$

Scar

$W T$
Guanosine diphosphate

GDP-GTP exchange factor

Axenic medium

Latrunculin A

Phosphoinositide 3-kinase

Phosphatidylinositol 4,5- biphosphate

Phosphatidylinositol 4,5- triphosphate

Phosphatase and Tensin homolog on chromosome ten

Ras binding domain

Suppressor of CAR (independent of its name, it controls the activity of polymerization nucleation promoting factors)

Wild type cells - Defined here as cells with no genetical mutation, or with mutations for fluorescent labelling only 


\section{Contents}

\begin{tabular}{lll}
\hline 1 & Introduction & 11
\end{tabular}

\begin{tabular}{lll}
\hline $\mathbf{2}$ & Background & $\mathbf{1 5}$
\end{tabular}

$2.1 \quad$ Dictyostelium discoideum as a model organism . . . . . . . . . . . . 17

2.2 Signaling network . . . . . . . . . . . . . . . . . . . . . . 18

$2.2 .1 \quad$ G-coupled receptors module . . . . . . . . . . . . . 20

2.2 .2 Ras module . . . . . . . . . . . . . . . . 20

2.2 .3 Phosphotidylinositol module . . . . . . . . . . . . . . 21

2.3 Actin cytoskeleton . . . . . . . . . . . . . . . . . . . 23

$2.3 .1 \quad$ D.d. LimE as a filamentous actin sensor . . . . . . . . . . . 26

2.3.2 Polymerization regulator Actin interacting protein 1 (Aip1). 27

2.3.3 Polymerization regulator Coronin $1 \mathrm{~A}(\mathrm{Crn})$. . . . . . . . . 30

$2.3 .4 \quad$ Cooperative effects of Coronin 1A and Aip1 . . . . . . . . . 32

2.4 Dynamical properties and time scales of the signaling cascade and actin cytoskeleton . . . . . . . . . . . . . . . . . . . 34

$2.4 .1 \quad$ Fast time scales, order $\sim 1 s \quad \ldots \ldots . \ldots . \ldots 35$

$2.4 .2 \quad$ Intermediate time scales, order $\sim 10 \mathrm{~s}$. . . . . . . . 35

$2.4 .3 \quad$ Slow time scales, order $\sim 100 \mathrm{~s} \ldots \ldots . \ldots 36$

2.5 Objective $\ldots \ldots \ldots \ldots \ldots$

3 Materials and methods $\quad 41$

3.1 Cell culture . . . . . . . . . . . . . . . . . . . . . . . . . . . . . . 42

3.2 Flow photolysis . . . . . . . . . . . . . . . . . . . . 43

3.3 Experimental set up . . . . . . . . . . . . . . . . . 43

3.3 .1 Soft lithography . . . . . . . . . . . . . . . . . . 46 


\section{CONTENTS}

3.3 .2 Caged compounds . . . . . . . . . . . . . . . . . . . . . 47

3.3 .3 Confocal microscopy . . . . . . . . . . . . . . . 47

3.4 Signal processing . . . . . . . . . . . . . . . . . . . . . . . . . . . 48

$3.4 .1 \quad$ Data retrieval . . . . . . . . . . . . . . . . . . . . . . . . 48

3.4 .2 Correlation analysis . . . . . . . . . . . . . . . . . . 50

$4 \quad$ Autonomous activity of the actin cytoskeleton $\quad 55$

4.1 Experimental protocol and measured parameters . . . . . . . . . . 57

4.2 Actin binding proteins and pseudopod extension . . . . . . . . . . 58

$4.3 \quad$ Period and phase distribution $\ldots \ldots \ldots \ldots$. . . . . . . . . . 62

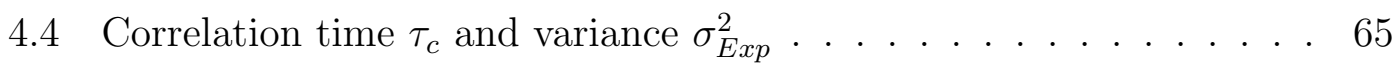

4.5 Origin of the statistical properties of actin oscillations. . . . . . . . 70

4.5.1 Close to an oscillatory instability with additive noise . . . . 70

4.5.2 Multiplicative noise and far from the oscillatory instability point . . . . . . . . . . . . . . . . 72

$4.5 .3 \quad$ Interpretation of both scenarios . . . . . . . . . 76

4.6 Transient responses . . . . . . . . . . . . . . . . . . . . . 78

4.7 Modeling long transients . . . . . . . . . . . . . . . . . . . . 79

$4.7 .1 \quad$ Phase portraits of different scenarios . . . . . . . . . . . . . 79

4.7 .2 Interpretations for the fast and slow time scales . . . . . . . 83

4.8 Chapter summary . . . . . . . . . . . . . . . . . . . . . . . . . . . . . . . 84

\begin{tabular}{|lll}
5 & Dynamical responses of actin regulating proteins & 89
\end{tabular}

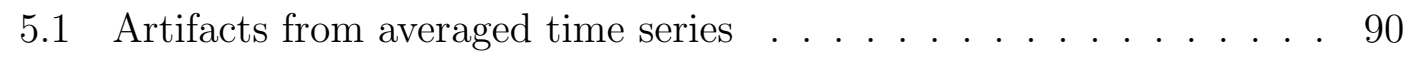

5.2 Data segregation for averaging . . . . . . . . . . . . . . . . . . 92

5.3 Wild type cells $\ldots \ldots \ldots$. . . . . . . . . . . . . . . . . . . 95

$5.3 .1 \quad$ Cell to cell variability in WT . . . . . . . . . . . . . . 98

$5.3 .2 \quad$ Symbolic dynamics . . . . . . . . . . . . . . . . . . 101

5.4 Knockout cells . . . . . . . . . . . . . . . . . . . . . . . . . . . . . . 104

5.4 .1 Cell to cell variability in Knockouts . . . . . . . . . . . . . 106

5.4 .2 Concavity . . . . . . . . . . . . . . . . . . . 108

5.4 .3 Concavity distribution . . . . . . . . . . . . . . 111

5.5 Interpretation . . . . . . . . . . . . . . . . . . . . . . . . 113 


\section{CONTENTS}

5.6 Chapter summary . . . . . . . . . . . . . . . . . . . 120

6 Conclusions and discussion 123

A Analytical and numerical calculations of correlation time $\tau_{c}$ and $\begin{array}{ll}\text { variance } \sigma^{2} & 129\end{array}$

A.1 Correlation time in multiplicative noise model . . . . . . . . . . . . 129

A.2 Numerical validation for short time series . . . . . . . . . . . . . . . 131

\begin{tabular}{ll}
\hline B Numerical simulations & 135
\end{tabular}

B.1 Figure 4.11 and A.1. . . . . . . . . . . . . . . . . . . . 135

B.2 $\quad$ Figure A.2 . . . . . . . . . . . . . . . . . . . . . . 136

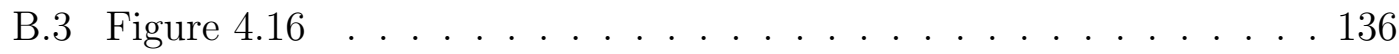

B.4 Figure 4.15, left . . . . . . . . . . . . . . . . . . . . 137

B.5 Figure 5.1 and $5.2 \ldots \ldots \ldots$. . . . . . . . . . . . . . . . 137

\begin{tabular}{lll}
\hline C Samples of self sustained oscillations and colored noise & 139
\end{tabular}

D Example of membrane retraction and oscillation 143 
CONTENTS 


\section{Chapter 1}

\section{Introduction}

It has been 12 years since Michael G. Vicker proposed that actin filaments in Dictyostelium discoideum propagate as a self organized reaction-diffusion wave [108, 109]. Several studies since then have endeavoured to quantify the formation of lateral waves in several organisms [27, 66, 86], showing the existence of characteristic spatiotemporal scales. During the developmental stage of Dictyostelium discoideum, this propagation can be induced by changing the external concentration of cAMP, resulting in the translocation from cytosol to cortex of several proteins with a characteristic time scale of one minute [32]. The development of flow photolysis [8] has brought the possibility of stimulating single cells with short pulses of one second by using photoactivated cAMP.

The mean cytosolic response of Dictyostelium discoideum cells to a single short pulse of cAMP is reminiscent to the dynamics of a damped oscillator. This concept is supported by the existence of a resonance frequency when the cells are stimulated periodically with short pulses [113, 114]. In addition, mutant cells lacking the regulators of the Arp2/3 complex (SCAR/PIR) show periodic actin polymerization cycles that suggests the existence of an oscillatory instability [51]. So even as the actin cytoskeleton is composed of 138 proteins, according to the Dictyostelium discoideum genome [30], current evidence shows that it behaves as a nonlinear oscillator with low degrees of freedom.

In this work we have continued to study the dynamical properties behind the previously observed responses and dynamical states of actin polymerization using 
Dictysotelium discoideum as a model organism. The first question we have addressed is the dynamical nature behind the observed self sustained oscillations. To gain information behind this process we have perturbed randomly single cells anticipating that some of them will show oscillatory activity. We discovered that self oscillating cells respond differently to external pulses of cAMP, some of them show a short transient response where the cell keeps oscillating afterwards, while others show a long transient response where the oscillatory activity ceases completely to then be recovered after $\sim 100 \mathrm{~s}$. The recovery is gradual suggesting that these polymerization cycles corresponds to limit cycle oscillations. In cells showing coherent oscillations, there are periodic pseudopod extensions that are correlated with the actin dynamics, showing the relevance of this process for understanding cell motility.

Mesoscopic systems are susceptible to noise given their interaction with the environment and for which the actin cytoskeleton is no exception. Standard fluctuations analysis has shown the possible existence of a decorrelation mechanism in these oscillations that depends on the signal variance. We have analyzed our data using the Stuart-Landau oscillator in its Langevin version, and with it we have constructed two different scenarios that can reproduce our experimental observations. Numerical and analytical calculations suggest one of them as the valid mechanism in which the majority of the observed oscillations are noise induced. Also it has been shown, using geometrical arguments in phase space, that this two component model cannot take into account the observed long transients. The physical interpretation of this result is the existence of a slow process that is quiescent during oscillatory actin polymerization, and when a cell is stimulated with external cAMP it becomes active terminating with the observed activity.

In the second part of this work we have studied the dynamics of actin polymerization along with its regulators, Aip1 and Coronin 1A. Single cAMP pulse stimulation experiments were performed in cells having fluorescent markers in these proteins, where we have characterized their translocation dynamics to the cell cortex. As mentioned the actin cytoskeleton has many components which details we ignore, therefore we have used a proposed method that give information on the possible effective interactions between them. It consists of transforming the observed responses into a sequence of symbolic dynamics that give the activating and 
inhibiting interactions between the observables [81, 82]. We have verified how the predicted interactions hold by studying the cAMP response of mutant cells missing either Aip1 or Coronin 1A. Our interpretation of the data led us to conjecture the existence of a component that is inhibited by the cooperative effect of Aip1 and Coronin 1A, when any of these two regulators are missing this hypothetical component enhances polymerized structures. 


\section{Chapter 2}

\section{Background}

The actin cytoskeleton constitutes the basis for cell motility and during its operation, several processes inside the cell are regulating its dynamics [85]. As a cell is crawling in its natural environment, it will be detecting external chemoattractant gradients, and it will incline its motion towards the source. For this to happen several components are in charge of guiding the directionality, which we denominate as the signaling system [100]. At the same time actin polymerization will be regulated by proteins such as Aip1 and Coronin 1A.

Figure 2.1 shows the ordering on how external signals of cAMP are biochemically transmitted to the actin cytoskeleton. The external cAMP molecules interact with the cell receptors, which at the same time interact with the components of the signaling system. The actin cytoskeleton by itself is an autonomous system, which receives information from the signaling system to regulate its activity [76]. In this work we are interested in learning the basic principles behind actin polymerization and regulation, and for that we study the physiological response of Dictyostelium discoideum to cAMP.

As also shown in the scheme of Figure 2.1, we control in experiment the amount of cAMP given to the cell and observe the activity of the actin cytoskeleton. We extract time series from those observations which will be used to analyze and interpret them. For an optimal data interpretation it is important to review what is known about the signaling system, since its time scales might be reflected in our observations. But also it is necessary for studying the dynamics of polymerization 
regulators Aip1 and Coronin 1A, to review what its known about them from in vitro and in vivo experiments performed by other authors.

This chapter will be organized as follows, in the first section details of the model organism Dictyostelium discoideum are reviewed. Next the details of the signaling system for this particular organism are discussed followed by a review of the actin cytoskeleton. A literature survey for Aip1 and Coronin 1A will be next in which the results from several in vitro and in vivo experiments from various organisms will be discussed. Finally a discussion on the time scales observed in these processes and the aim of the project are discussed. The introduction and background of experimental and analysis techniques will be left for Chapter 3 - Materials and methods, leaving this chapter exclusively for the review of the current knowledge about the molecular biology and dynamics behind the regulation of actin polymerization.

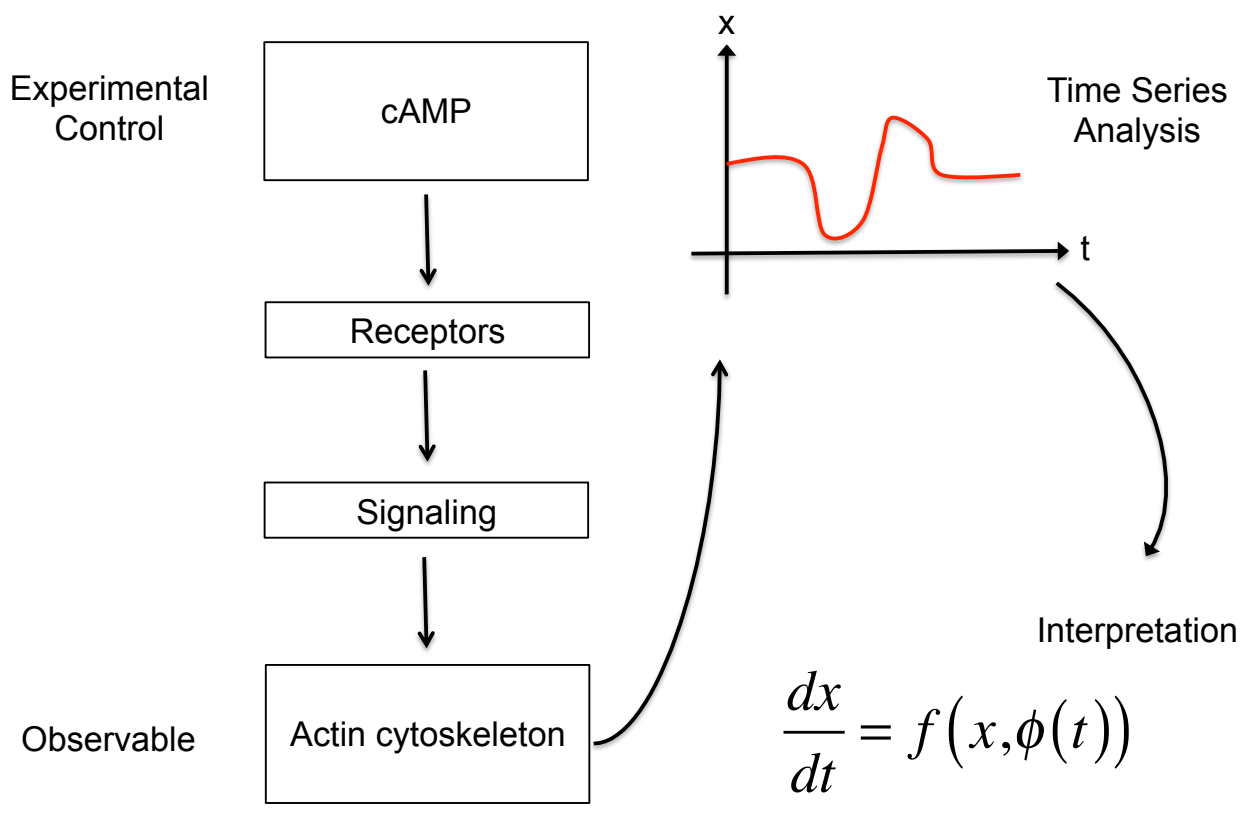

Figure 2.1: Scheme showing how cAMP signals are transmitted to the actin cytoskeleton. In experiment we control the external amount of cAMP and observe the activity of the cytoskeleton, time series are then retrieved and analyzed. 


\subsection{Dictyostelium discoideum as a model organ- ism}

Dictyostelium discoideum is an amoeba that lives in the soil and has a solitary life feeding on bacteria [54]. If a colony of cells is kept without nutrients long enough, the cells will aggregate and form a multicellular organism increasing its chances for survival. This self organized process happens as the interplay of single cell production of cyclic adenosine monophosphate (cAMP) and the detection of resulting waves which feedbacks into its production [37]. This interaction results in cAMP spiral waves patterns reminiscent to the ones observed in excitable media [22]. After two hours from the formation of spiral waves, the cells crawl towards the center of the spiral which they localize by detecting cAMP gradients [94].

This particular ability to detect external chemical gradients and crawl towards them is denominated as chemotaxis, and in this work we are interested in studying cells during the chemotactic stage. Each cell has a myriad of different proteins assigned for the processes of detection, motility and polarization [100. With detection it refers to the before mentioned ability to detect the external cAMP gradients, which is due to the expression of cAMP receptors (CAR) at the cell wall. As the cell detects chemoattractant gradients it crawls towards them, and the process is denominated as motility. While polarization refers to the elongation of cell shape with a well defined front, where the dissociation rates at the CAR are higher and protrusions called pseudopods are more likely to be created. These three processes have been observed to occur independently, as detection can occur in non motile cells while motility can happen in non polarized cells and without any chemoattractant [100].

In this work we are interested in studying the machinery in charge of cell motility, Figure 2.2 shows the basic components for this process. Motility is complemented by contraction in the back assisted by the motor protein Myosin [69]. While pseudopods are created by the polymerization of filamentous actin (FActin), which dynamical states and regulation are the subject of this work. It has been shown that a physiological polymerization response is induced by external stimulation of cAMP [114], this stimulation must be processed by several components before it acts on the cytoskeleton [100]. This signaling network is in 


\subsection{SIGNALING NETWORK}

charge of bridging the receptors and the actin cytoskeleton, and will be reviewed next .

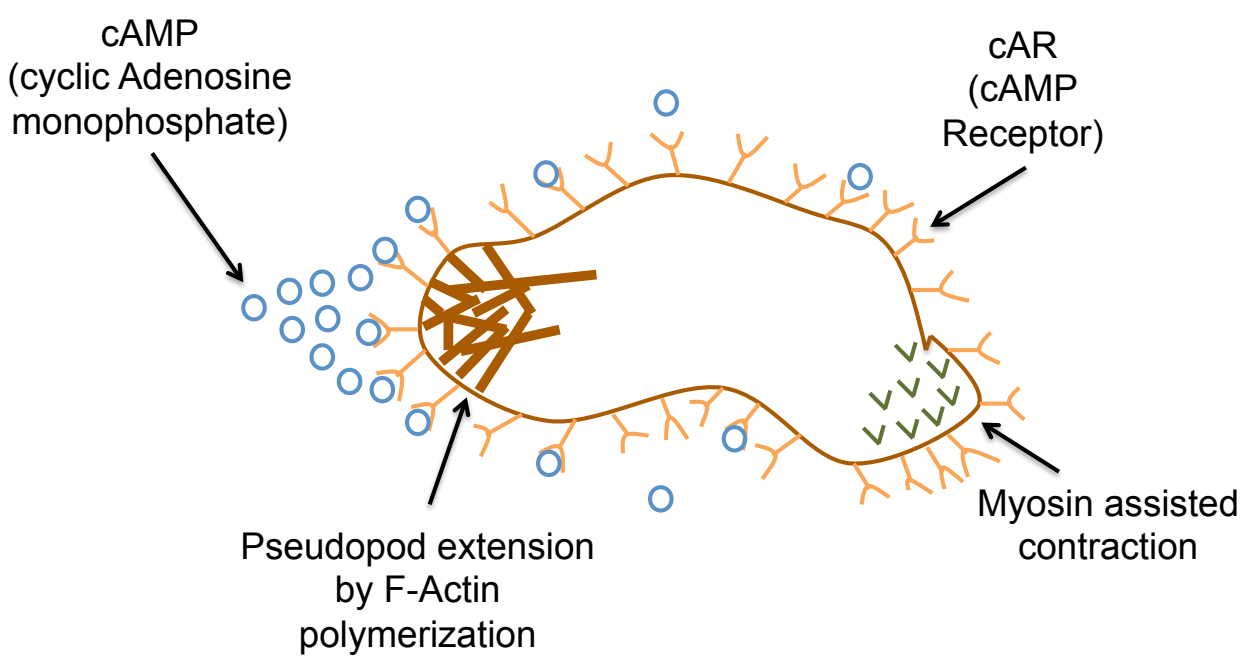

Figure 2.2: Basic components of the chemotaxis machinery of Dictyostelium discoideum, the cell reaches the chemoattractant source by extending pseudopods and contracting its back.

\subsection{Signaling network}

Dictyostelium discoideum has an intrinsic machinery to detect and localize an external source of cAMP. This machinery consists of proteins arranged in different modules which processes these external signals, and consequently the cell will bias its motion towards the source. The components of the chemotactic machinery can exhibit autonomous activity, or can be activated by external addition of chemoattractant. Figure 2.3 is a representation of one of the most studied biochemical signaling pathways for chemotaxis. The pathway shown in Figure 2.3 is an oversimplification of the actual pathway [100], but contains the essential proteins for chemotaxis in which the links represent their indirect interaction. This pathway is divided into three stages: the first being the cAMP receptor module in charge of detecting the external concentrations of cAMP. Following is the RasC module (mentioned as Ras in the remaining part of the section) which reflects the internal 


\subsection{SIGNALING NETWORK}

compass of the cell, and finally the phosphatidylinositol (PIP) module which bias the direction of actin polymerization. The components of this pathway reflects the processes of sensing, polarity and motility observed in chemotaxing cells. This picture might not be completely accurate since there are possible feedbacks between different modules, but several experimental evidence supports this approximation. They consist of observing the sequential activation of components along with the repercussions when one is genetically removed. Here some results from several studies are reviewed.

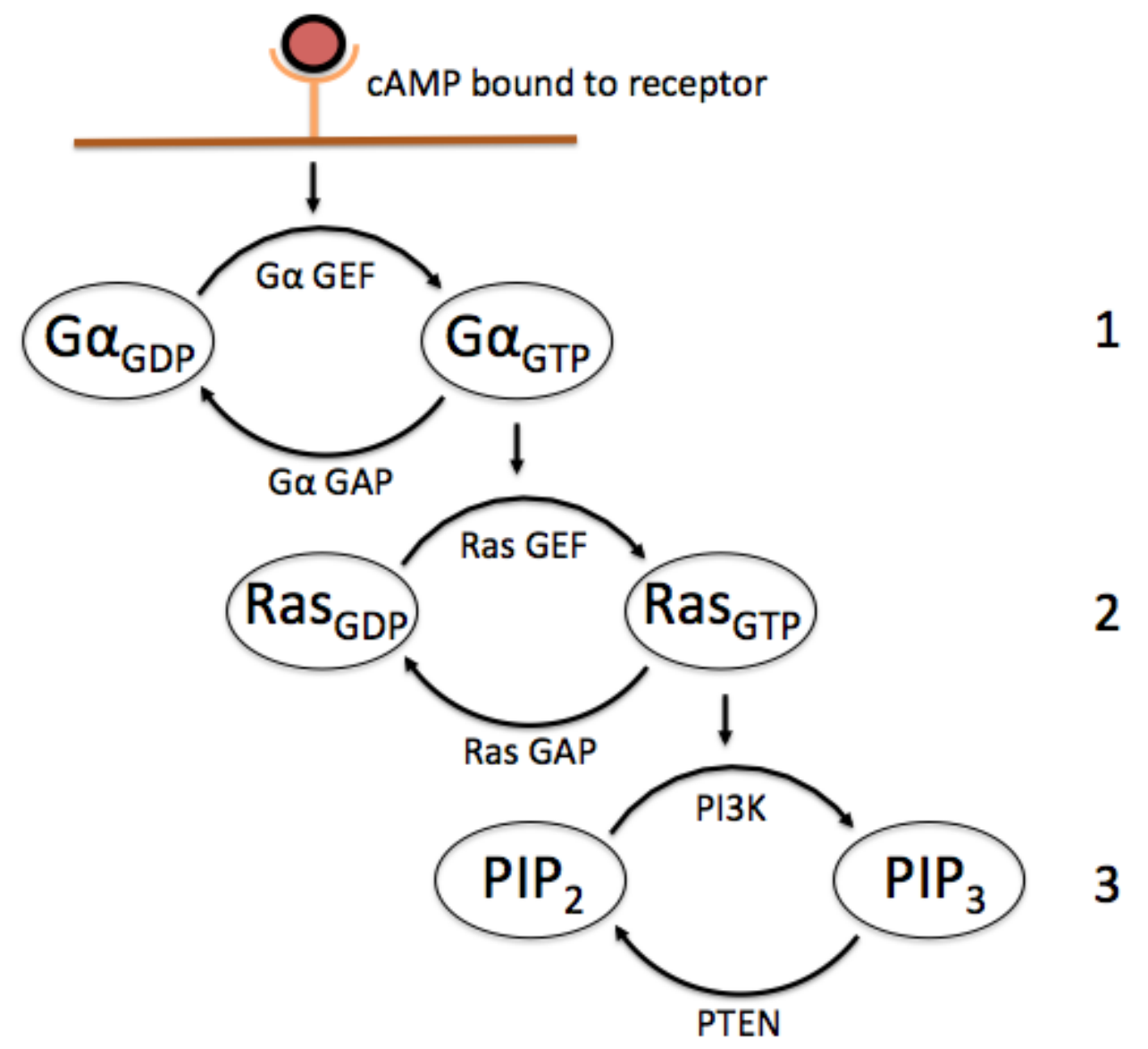

Figure 2.3: Essential components of the Dictyostelium discoideum signaling cascade for biasing the motion towards cAMP sources. The cascade is divided in three modules: 1- CAR module, 2 - Ras module and 3-PIP module. 


\subsection{SIGNALING NETWORK}

\subsubsection{G-coupled receptors module}

The first step in the signal processing is the binding of cAMP with its receptors (CAR). Dictyostelium discoideum has four different cAMP receptors, CAR 1-4, in which the disruption of CAR 1 and CAR 3 completely terminates with the ability of a cell to chemotax [100]. CAR belong to a class of receptors called G-coupled receptors, these are embedded in the cell wall with half of the protein standing outside the cell and the other half inside. Inside the cell wall next to the receptor, there is a protein complex with three subunits called $\mathrm{G} \alpha, \mathrm{G} \beta$ and $\mathrm{G} \gamma$ [67]. The $\mathrm{G} \alpha$ subunit is a GTP-ase, which is a molecule with guanosine diphosphate (GDP) attached to it during its inactive state. When a cAMP molecule binds to the receptor the $\mathrm{G} \alpha$ subunit is activated by the exchange of $\mathrm{G} \alpha$-GDP $\rightarrow \mathrm{G} \alpha$-GTP, mediated by the GDP-GTP exchange factor (GEF). Upon activation the protein subunits detaches from the cell wall and splits into two groups, one tandem being of $\mathrm{G} \beta$ and $\mathrm{G} \gamma$ and the other being of G $\alpha$ and GTP. When the ligand disengages from the receptor, a GTP-ase activating protein (GAP) hydrolyzes $\mathrm{G} \alpha$-GTP $\rightarrow \mathrm{G} \alpha$-GDP and $\mathrm{G} \alpha, \mathrm{G} \beta$ and $\mathrm{G} \gamma$ return to their previous state [67]. These subunits are responsible for transmitting the information to other modules of the signaling network [100]. The kinetics of these proteins differ between the ones at the front of a crawling cell towards a chemoattractant compared to the ones at the back [106]. In polarized Dictyostelium discoideum, it has been shown that the ligands have a shorter residence time in the receptors at the pseudopods compared to the ones in the tail. By preparing the cell membranes with GTP it was shown to induce a decrease in ligand binding lifetime [106], while preparing them with GDP induces the opposite effect. Therefore the difference between front/back with activated/deactivated receptors reflects the polarity of chemotaxing cells.

\subsubsection{Ras module}

The information transmitted by the G proteins is received by secondary messengers in cascade manner. In chemotaxis the essential secondary messengers associated with the activation of the actin cytoskeleton (which is reviewed in the next section) are Ras C and Ras G [100]. The Ras proteins are also GTPases that have an inactive state associated with a GDP molecule embedded to it which is activated 


\subsection{SIGNALING NETWORK}

via its own Ras-GEF [67]. Interestingly the spatial distribution of activated Ras in the cell membrane reflect the external spatial cAMP concentration profiles imposed to it. In a series of experiments the cells were imposed an external cAMP gradient in which the protein RBD (Ras binding domain), which diffuses to the cell cortex when Ras is activated, was fluorescencently labeled [53]. RBD translocated to the cell cortex and reflected the external gradient profile. The mapping of external cAMP profiles by Ras proteins is independent of the cytoskeleton as it was shown by treating the cells with Latrunculin A (Lat A, a toxin that inhibits the polymerization of the actin cytoskeleton) [53]. In a detailed study of the initiation of chemical sensing, it was shown that Ras reflected different stages of sensing activation. The cells responded around one second after stimulation by a generalized activation of Ras all over the cell cortex, then at $20 s$ the cell adapted to a state in which Ras deactivated to their previous levels, followed by a symmetry breaking at $40 s$ directed in which Ras was directed towards the source followed by a confinement of Ras to a smaller crescent [57].

\subsubsection{Phosphotidylinositol module}

Sequentially the activity of RasG activates the phosphatidylinositol module (denoted with 3 in Figure 2.3p important for the directionality of pseudopod extension [49]. In a similar manner as with the GTP-ases G $\alpha$ and Ras, the phospholipid of this module has two states for promoting pseudopod extension: inactive, called phosphatidylinositol 4,5- biphosphate (PIP2) and active, called phosphatidylinositol 3,4,5- triphosphate (PIP3). The transitions between these two states are mediated by two enzymes, the transition PIP2 $\rightarrow$ PIP3 is mediated by Phosphoinositide 3-kinase $(\mathrm{PI} 3 \mathrm{~K})$ and the transition PIP3 $\rightarrow$ PIP2 by the phosphatase and Tensin homolog on chromosome ten (PTEN) [100].

The thought of an induced transition of PIP2 into PIP3 by the activity of Ras is supported by a couple of experimental observations. The first one from [93], consists in the observation of sequential activation between the proteins RBD and PH-CRAC which translocates from cytosol to the cortex when Ras and PIP3 are activated respectively. In these experiments it was observed that PI3K translocated from the cortex apparently at the same time as RBD, therefore for the 


\subsection{SIGNALING NETWORK}

scheme in Figure 2.3 to be fulfilled the response time of PI3K must be very fast compared to the one for RBD. Usually enzymes has fast dynamics and could justify this [67], another possibility is that of a positive feedback loop between Ras and PI3K that causes the two labels to correlate. In another set of experiments the activity of the Ras-GAP was disrupted, which is achieved by the genetical removal of nfaA. In these experiments the second translocation of RBD from cortex to cytosol takes significantly longer than in the wild type, meaning that the transition Ras-GTP $\rightarrow$ Ras-GDP was slowed down. In consequence to this mutation the kinetics of PIP3 also slowed down dramatically suggesting that it is due to the slowing down of the Ras dynamics [123].

The phospholipids PIP2, PIP3 and the enzymes PI3K and PTEN also encode the spatial profile of cAMP. In [53] it is shown that in cells treated with Lat A, PI3K translocates to the front side where the concentrations levels of cAMP are high while PTEN goes to the rear where the concentrations levels are low. This spatial localization is also been observed in cells crawling in cAMP gradients [89]. All the molecules of Figure 2.3 integrate the external changes of cAMP, but Ras and PIP3 adapt by returning to the previous basal level after some time. Though in [75] it is shown that the amount of cells showing spatial transient activations of PIP3 increases with the amount of cAMP background concentration. Also these cells have shown persistent domain formations of PIP3 that traveled around the cell cortex, these domains are not present when there is no background cAMP.

Cells with a knockout in PI3K can still chemotax but disruption of PTEN has severe phenotypes. The response kinetics of PTEN $\Delta$ cells are similar to those of nfaA $\Delta$ cells in which the translocation back to the cytosol took significantly longer than compared to wild type. These cells while chemotaxing extended three or more pseudopods in random directions and as a consequence some of the movements were in the opposite direction from the chemical source and its trajectory was more circular compared to the the straight trajectories performed by the wild type [49]. Therefore it is thought that PTEN is necessary for the proper regulation of pseudopod extension in Dictyostelium discoideum making it an essential link between the signaling pathway and the actin cytoskeleton. 


\subsection{ACTIN CYTOSKELETON}

\subsection{Actin cytoskeleton}

The cell cytoskeleton is essential for the mechanical properties, motility and endogenous organization of an eukaryotic cell. It is composed of three parts: the microtubules, which are straight hollow rods that are capable of organizing and moving organelles. Following are the intermediate filaments which give mechanical properties such as shear stress resistance, and finally the actin cytoskeleton which provides the basis for shape deformation and motility [1].

As we are concerned with the properties of cell motility, our study is centered in the dynamical properties and regulation of the actin cytoskeleton. It consists of a network of polymerized filaments spread all over the cell volume. A single filament has a persistence length $10 \mu \mathrm{m}$ which is comparable with the typical cell length, and therefore they are basically straight inside the cell volume [10]. The filamentous actin is constructed with the polymerization of basic units called gactin monomers. Each monomer has a weight of $42 \mathrm{kDa}[10$ ] and half of the actin inside the cell is in this state [13.

The structure of the cytoskeleton is complicated and consists of different structural organizations identified as: branched networks, crosslinked networks, parallel bundles and antiparallel contractile bundles. Before discussing the role of specific structures it is useful to visualize a heuristic picture of a small actin filament network with constant length (Figure 2.4). As the cytoskeleton is a structure far from thermodynamic equilibrium, the steady state of this single filament would consists of a constant polymerization and depolymerization process [17]. This polymerization/ depolymerization process is controlled inside the cell by a myriad of actin binding proteins, in Dictyostelium discoideum these proteins falls at least into 7 classes being in total 138 [30]. Though the observed polymerization dynamics can be observed in vitro by adding the following basic compounds (Figure 2.4): actin, actin depolymerization factor(ADF)/cofilin, a capping protein, Arp2/3 complex, an activator of the Arp2/3 complex (like Scar for example) and profilin [85].

The monomeric and filamentous actin are ATP-ases that can be in either one of these states: ATP-actin, ADP-actin and a transient hydrolysis state ADP+Pactin. The single actin filaments are polar, on one end the association rate is higher where actin monomers are recruited and elongates. This end of fast elongation is 


\subsection{ACTIN CYTOSKELETON}

called the barbed end (denoted as red filaments in Figure 2.4). On the other side of the filaments the dissociation rates are higher and therefore disassembly is promoted, and this part is called the pointed end. The following table summarizes the association and dissociation rates on both sides as measured in an actin only assay (Table 2.1) 84].

\begin{tabular}{ccccc} 
& \multicolumn{2}{c}{ ATP-actin } & \multicolumn{2}{c}{ ADP-actin } \\
\cline { 2 - 5 } & Barbed & Pointed & Barbed & Pointed \\
\hline$k_{+}\left(\mu M^{-1} s^{-1}\right)$ & 11.6 & 1.3 & 3.8 & 0.16 \\
$k_{-}\left(s^{-1}\right)$ & 1.4 & 0.8 & 7.2 & 0.27 \\
\hline
\end{tabular}

Table 2.1: Association and dissociation constants for actin monomers taken from Pollard, 1986 84.

The association constants $k_{+}$depends linearly on the amount of available actin monomers, it can be noticed that the association rate for the barbed end is roughly four times higher for ATP-Actin compared to ADP-Actin. On the contrary the dissociation rate is roughly 6 times higher for ADP-Actin on the barbed end, therefore the polymer on the barbed end consists of an overwhelming majority of ATP actin. As an actin polymer grows, the components in the filament ages and they undergo an hydrolysis process transforming Actin-GTP $\rightarrow$ Actin-GDP. Most of the actin present in the actin filament is in the intermediate state $\mathrm{ADP}+\mathrm{P}$-Actin but at the back of the filaments, where the older filaments reside, the components are already in the hydrolized ADP-Actin state. It is noticeable that in the back the dissociation and association rates are higher for ATP-actin. In vitro [9] and in vivo [10] the actin in the pointed end is in the ADP state, therefore other proteins are contributing to maintain this state (Figure 2.4). The Arp2/3 complex inhibits the association and dissociation in the pointed end contributing to the maintenance of filament polarity [73].

Nucleation of actin polymers is thermodynamically unfavorable, but once oligomers are formed spontaneous nucleation can occur if the concentration is above a critical concentration [10]. The ratio $k_{-} / k_{+}$gives the critical concentration for spontaneous nucleation which corresponds to the value $0.1 \mu M$ [85]. Therefore to avoid spontaneous nucleation actin monomers are complexed with profilin, which se- 


\subsection{ACTIN CYTOSKELETON}

questers the actin monomers individually. In this process the Arp2/3 complex plays the role of a nucleation promoting factor (NPF), that controls the nucleation of filaments whenever is needed. The nucleation happens on the sides of preformed filaments making an angle of $70^{\circ}$, making dense branched networks [73]. The majority of the filaments are formed on the sides of ADP-actin or ADP+P-actin and the absence of the Arp2/3 complex results in very long filaments [9], which consequently the resulting actin network is not dense and affects the mechanical properties of cells [10]. The Arp2/3 complex by itself is not an effective nucleator and needs to be activated by proteins such as Scar (suppressor of cAMP receptor, originally discovered in D.d.), that belongs to the family of WASP (Wiskott-Aldrich syndome protein). Another important factor for the creation of dense networks are the capping proteins, these proteins inhibit the growth of filaments at the barbed end [10].

To have effective cell motility the actin filaments must be dismantled to recycle the monomeric actin pool and polymerize at different cell sites. The intrinsic depolymerization kinetics of actin filaments would not be enough to account for the observed actin turnover times observed in vivo. A set of proteins are in charge in dismantling these filaments by severing them into small pieces, the most studied protein in this context is ADF/Cofilin [85]. Although it has been found that the proteins of Figure 2.4 have specific roles and are necessary to reproduce the observed polymerization and depolymerization kinetics in vitro, there is also a synergy between them that regulate their operation. A combination of cofilin, capping protein and arp $2 / 3$ complex is necessary for the optimal branching observed in experiments [9].

One of the objectives of this work is to study the role of two different proteins known to be important for the regulation of actin turnover, Aip1 (actin interacting protein 1) and Coronin 1A (Crn). In several in vivo and in vitro experiments it has been shown that these two proteins interact and regulate with the basic components of the cytoskeleton [70, 77, 18, 63, 36, 59, 104, 14, 59]. In the following subsections a survey of the observations and conclusions of several studies determining the roles of Aip1 and Coronin 1A will be reviewed. But first the indirect marker used to monitor the activity of filamentous actin, LimE, will be discussed. 


\subsection{ACTIN CYTOSKELETON}

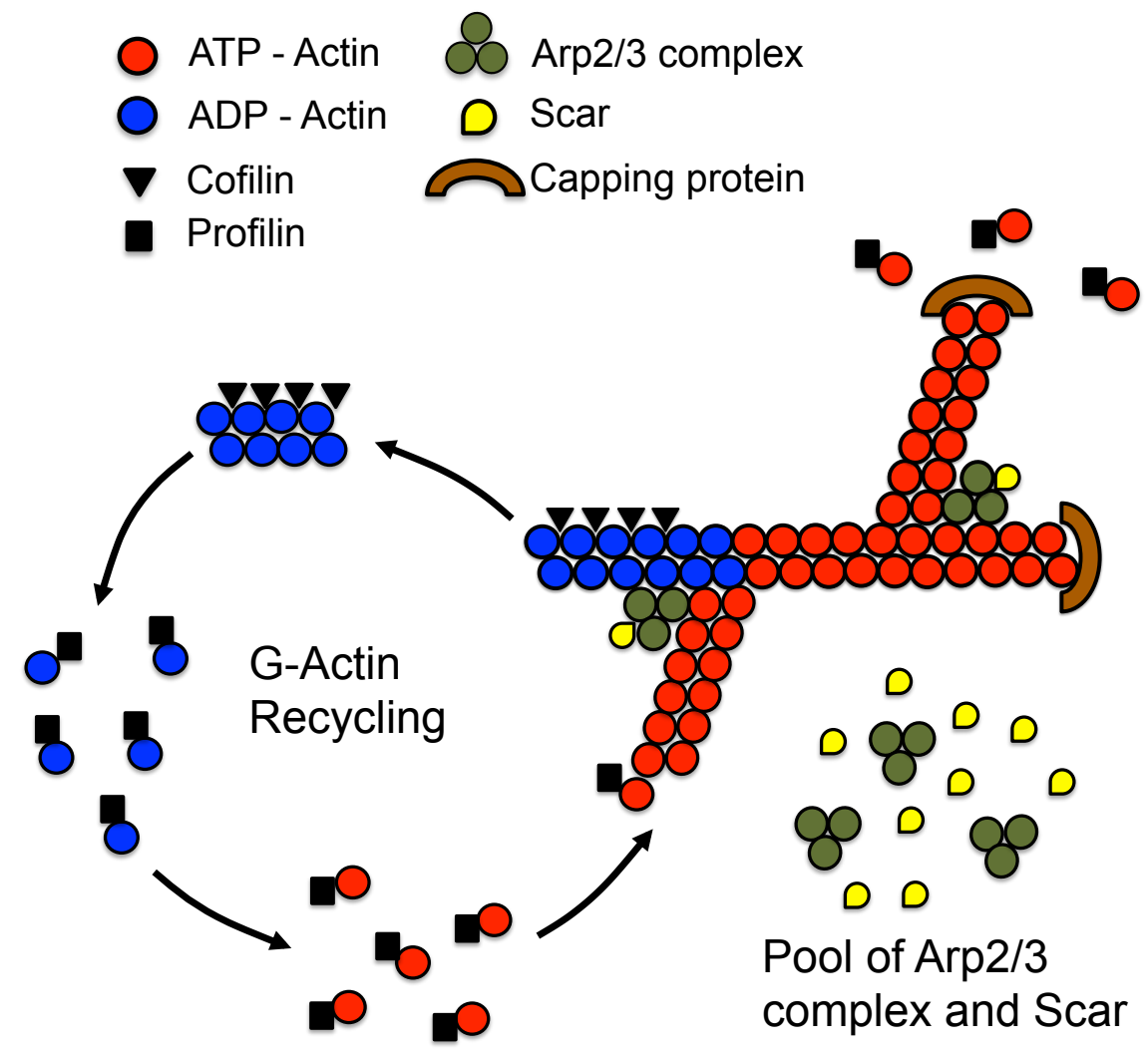

Figure 2.4: Schematic of the minimum amount of components to reproduce actin filaments treadmilling in vitro.

\subsubsection{D.d. LimE as a filamentous actin sensor}

Fluorescent labeling offers a practical way to study the dynamics of the cytoskeleton, its components can be followed by either addition of external substances (such as antibodies and toxins), labeled monomers or genetical modification. In Dictyostelium discoideum a strain which permanently produces a fusion of actin and green fluorescent protein was developed to track the polymerization dynamics of the actin cytoskeleton [115]. Control measurements with cells labeled with phalloidin-red and actin - GFP were performed to monitor their spatial distributions. Phalloidin-red labels selectively filamentous actin only and therefore these cells have a high fluorescence at the edges, the cell cortex, while the cytosol remains dark. In these experiments it was shown that the high fluorescence patterns 


\subsection{ACTIN CYTOSKELETON}

of phalloidin-red and actin-GFP matched at the edges in the cortical region, but actin-GFP could still be found in the cytosol because filamentous and monomeric actin are labeled in the same way. Also the observed polymerization dynamics in cells slows down once GFP is fused directly into actin, an alternative available is the direct labeling of actin with a small peptide called Lifeact that does not interferes with polymerization dynamics [90].

Half of the actin present in a cell is in the monomeric stage and therefore interferes with the imaging of single actin filaments. In Bretschneider et al. [13], it was concluded that fusing LimE with GFP was a suitable genetical modification to follow actin polymerization. Figure 2.5 shows an electro micrograph taken from cells with inmunogold labeling in either Arp2/3-GFP (panels A, B and C) or LimE $\Delta$ coil-GFP [95] (panels D,E, and F), it can be seen that Arp3 and LimE translocates to sites where actin filaments are present making them suitable candidates to follow filamentous polymerization. Though the Arp2/3 complex colocalizes in regions with high density actin clusters, LimE is distributed uniformly along in the actin network. This observation led the authors to conclude that LimE was a a label suitable for tracking actin polymerization.

\subsubsection{Polymerization regulator Actin interacting protein 1 (Aip1)}

As explained in the previous section the dynamical processes of actin treadmilling can be described by the interactions between actin, cofilin, capping proteins and nucleation promoting factors like profilin. In vitro experiments fail to reproduce the observed actin turnover (disassembly) rates found in vivo [78]. Therefore additional regulators promoting and inhibiting the components of the cytoskeleton are needed. Cofilin, responsible for the severing of filaments, was found to have its activity enhanced or complemented by other proteins. By genetical dissection it is found that Actin interacting protein 1 (Aip1) has binding sites to interact with F-actin and cofilin [20, making it an ideal candidate involved in the regulation of severing.

The absence of Aip1 in Dictyostelium discoideum results in different phenotypes, cytokinesis is slowed down by $36 \%$ compared to wild type. The growth 


\subsection{ACTIN CYTOSKELETON}

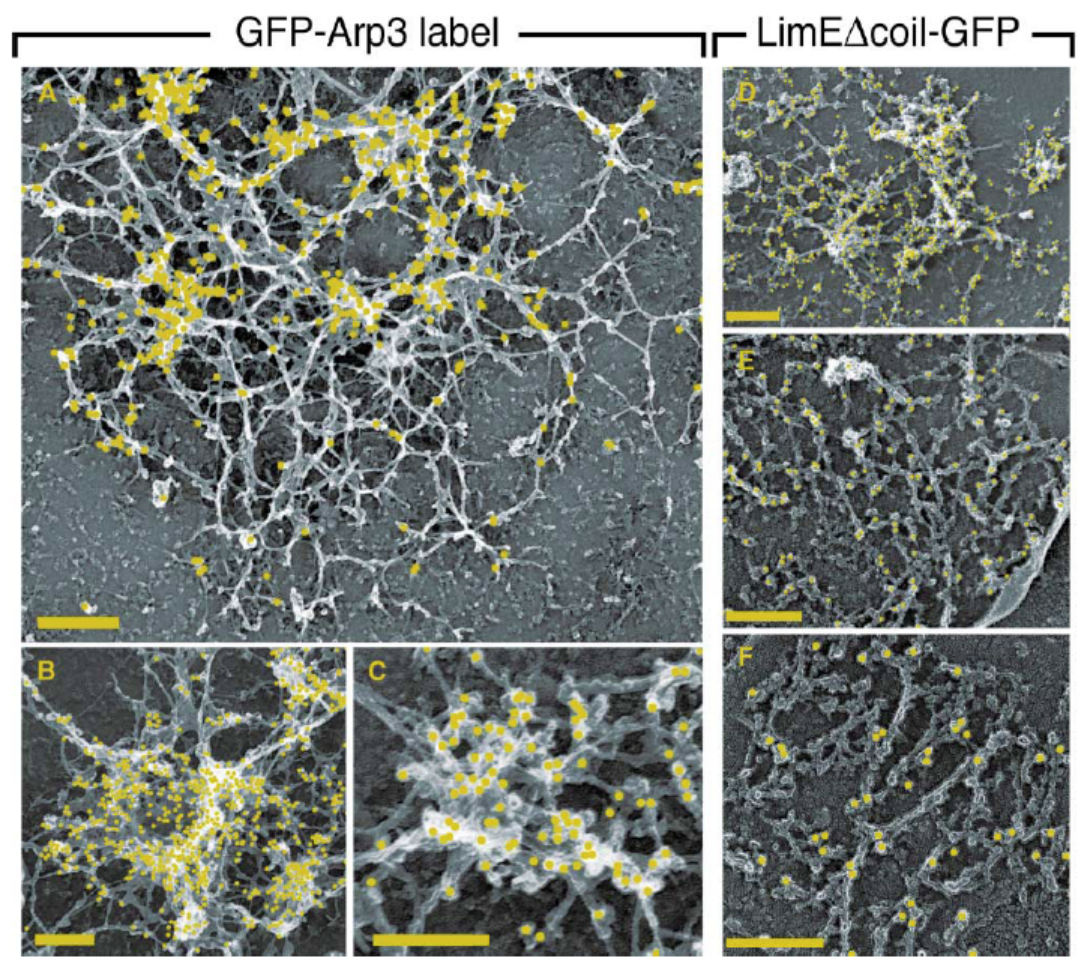

Figure 2.5: Pictures taken with an electron microscope comparing the distribution of GFP-Arp3 and LimE $\Delta$ coil-GFP from Bretschneider et al. 2004 [13]. It can be seen that GFP-Arp2/3 localizes preferentially in F-Actin clusters while LimE is distributed over F-Action therefore it is used as a polymerization sensor. 


\subsection{ACTIN CYTOSKELETON}

doubling time is prolonged to $12 \mathrm{~h}$ compared to the $8 \mathrm{~h}$ of the wild type, cell velocity of vegetative cells is reduced from $3.7 \mu \mathrm{m} / \mathrm{s}$ to $1.7 \mu \mathrm{m} / \mathrm{s}$, and the formation of phagocytic cup takes from inception to engulfment of a yeast particle 70-120 s compared to the 20-40 s of the wild type [56]. In all of these activities the cytoskeleton is essential and shows that disruption of Aip1 slows down the actin dynamics in Dictyostelium discoideum.

In Saccharomyces Cerevisiae (SC) long actin tails arrested to the endocytic machinery results from the deletion of the Sla2 protein, in this case it is found that Aip1 co-localizes with cofilin. In the presence of Lat A, remnant polymerization can still be observed in these tails. This polymerization was reasoned to rely on the presence of species that are not sequestrable by Lat A, thus actin oligomers [77]. This hypothesis was tested in an in vitro assay made from extracts from Sla2 $\Delta$ cells: in the first test, actin was kept constant and cofilin varied from 0 to $2.0 \mu \mathrm{M}$. Western blots have shown that actin monomers had a fold increase up to 1.5 times compared to its initial concentration, while dimers, trimers, tetramers and pentamers increased up to 3.5, 3.0, 2.5 and 2.0 times from their initial concentration, respectively. When Aip1 is added to the assay, the monomers increases 3.0 times from their initial concentration and dimers increased 2.5 times, while other species decreased in their number. Therefore it was hypothesized that Aip1 is important for the maintenance of actin monomers needed for polymerization.

It has been observed that the distribution of other actin capping proteins is affected by the genetical removal of Aip1 in Sla2 $\Delta$ SC cells [70]. The distribution of capping protein Cap2, has its maxima at the front of actin tails where the majority of ATP rich actin filaments reside. On the contrary Aip1 has its maxima at the back where ADP rich actin resides. Once Cap2 is removed from the cell, the maxima of Aip1 distribution shifts towards the front by $0.13 \mu \mathrm{M}$ suggesting that more binding sites are available to Aip1. Since Aip1 mainly bind to ADP actin this suggests that Cap2 is restricting the binding places for Aip1. While removal of Aip1 redistributes Cap2 to the whole region of actin tails. There is mutual inhibition between Aip1 and Cap2 and therefore Aip1 indirectly regulates the capping of actin filaments by restricting Cap2 to the ATP rich area.

Figure 2.6 summarizes these observations interpreted for a treadmilling actin filament: in a wild type cell there are two sources for polymerization. In the first 


\subsection{ACTIN CYTOSKELETON}

path of ( Figure 2.6, left ; 1,2,3,4) the actin filament is severed into oligomers but fails to be converted into single monomers by cofilin alone. Profilin then binds into the oligomer which consequently changes its nucleotide state, and after that the oligomer can be promoted for actin binding. In the alternative path $(1,2,5,6$; Figure 2.6 , left) the acting oligomer breaks into single monomers and after nucleotide exchange by profilin they are ready to bind to the barbed end of the filament. For the case when Aip1 is missing (Figure 2.6, right), the most likely path to happen is the first one since Aip1 is responsible for the fast dissociation into monomers. Also the capping protein Cap2 can be distributed in either pointed and barbed end of the filament since Aip1 is not present to restrict its position to the barbed end. Therefore with the current observations Aip1 is in charge of restoring the pool of actin monomers and the indirect spatial regulation of capping proteins.

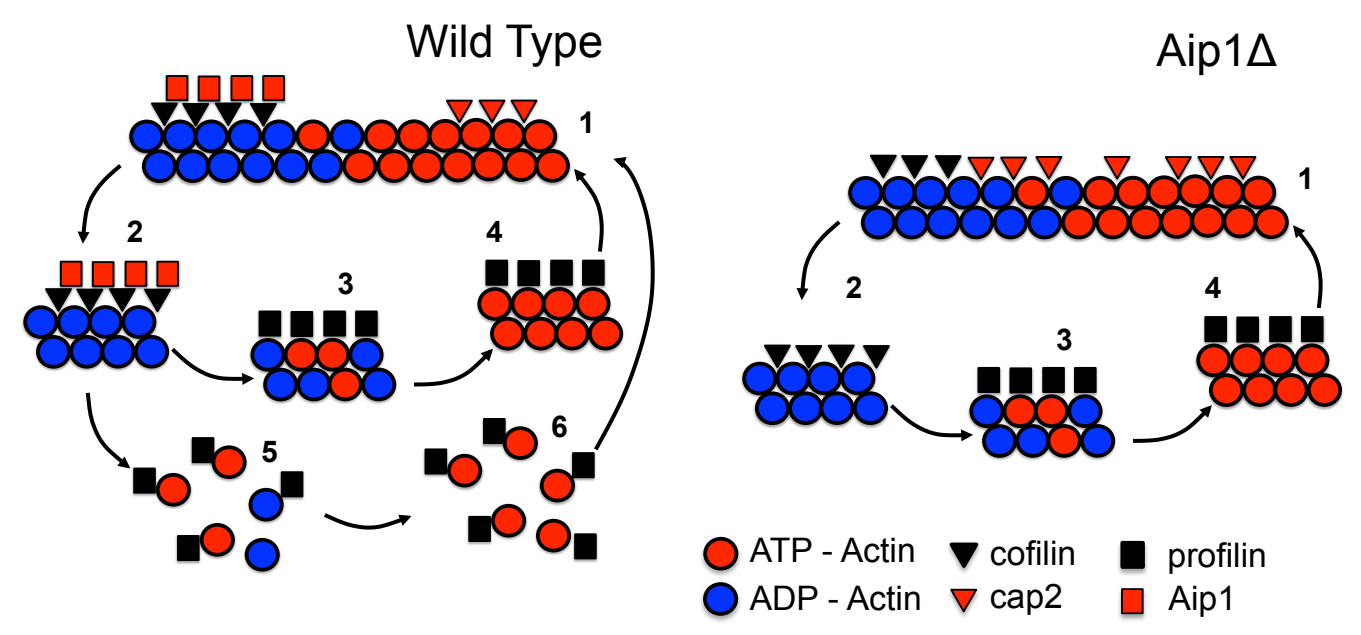

Figure 2.6: Visualization of Aip1 role in actin treadmilling. In wild type cells the severed actin components can turn into monomers due to the presence of Aip1, and in its absence result in polymerization with oligomers.

\subsubsection{Polymerization regulator Coronin 1 A (Crn)}

Coronin $1 \mathrm{~A}$ is a $49 \mathrm{kDA}$ actin binding protein discovered in Dictyostelium discoideum, its name comes from its association with the crown-shape surface projections during its growth phase [23]. Its absence results also in a variety of motility 


\subsection{ACTIN CYTOSKELETON}

and developmental phenotypes. Cells show slow motility in buffer of $3 \mu \mathrm{m} / \mathrm{min}$ and are defective in cytokinesis with similarities to Myosin II $\Delta$ cells [24]. These cells are also multinucleated, their development into mature fruiting bodies takes $30 \mathrm{~h}$ and phagocytosis is reduced, as has been shown by measuring the internalization rate of yeast and E. coli [96].

In Dictyostelium discoideum it has been shown that Coronin 1A indirectly regulates the levels of Myosin II [99]. These facts have shown that Coronin 1A is important for the regulation of the cytoskeleton, and several experiments have given insight on their dynamics at the molecular level. Also it has been shown that cells fail to initiate their developmental program when Coronin $1 \mathrm{~A}$ is missing, by addition of external cAMP the cells restore their developmental program [110].

In Gandhi et al. [36], in vitro and in vivo experiments with yeast were performed to test the binding affinity of Coronin $1 \mathrm{~A}$ in dependence to the nucleotide state of F-actin. The in vitro assays from yeast extracts have shown that when cofilin is present, the disassembly rate for ATP-actin increases 4.5 times while ADP-actin increases 3 times. This shows that ATP-actin becomes more sensitive to due to the presence of cofilin. The effect is illustrated in Figure 2.7 a where oligomers are produced from both sides and the pointed end is larger since it less sensitive to severing. With the addition of Coronin 1A into the assay, ATP F-actin recovered its stability to the same levels as if cofilin was not present. Then it is suggested that Coronin 1A has the role of stabilizing the barbed end against cofilin in vivo.

On the contrary the addition of Coronin 1A to the ADP-actin+cofilin assay increases the disassembly rate 9 times, suggesting that cells takes advantage of this effect for fast actin turnover rates. In vivo observations of actin tails have shown that the distribution of cofilin changed when Coronin 1A was knocked out, and it overlapped with that of Abp1 (actin binding protein1, an alternative actin marker). This redistribution indicates that cofilin was not confined to specific nucleotide sites of the actin filaments anymore, showing that the in vitro observations are relevant in the actin dynamics of living cells.

Also it has been observed that Coronin 1A interacts with the Arp2/3 complex [18]. A well characterized effect, is that of a Coronin $1 \mathrm{~A}$ concentration dependent switch for promoting actin polymerization [63]. In an actin+Arp2/3 


\subsection{ACTIN CYTOSKELETON}

complex assay the concentrations of Coronin 1A were varied from 0 to $100 \mathrm{~nm}$. The polymerization rate increases monotonically from 4 to $8 \mathrm{nM} / \mathrm{s}$. After that, a critical point is reached and further increase results in a monotonic decrease in polymerization up to to $1 \mathrm{nM} / \mathrm{s}$. Figure $2.7 \mathrm{~b}$ ) shows this effect in which at high Coronin 1A concentrations the Arp2/3 complex nucleation and branching is inhibited, while branched networks will be present as long as Coronin 1A is below the critical concentration (Figure 2.7 c).

a)
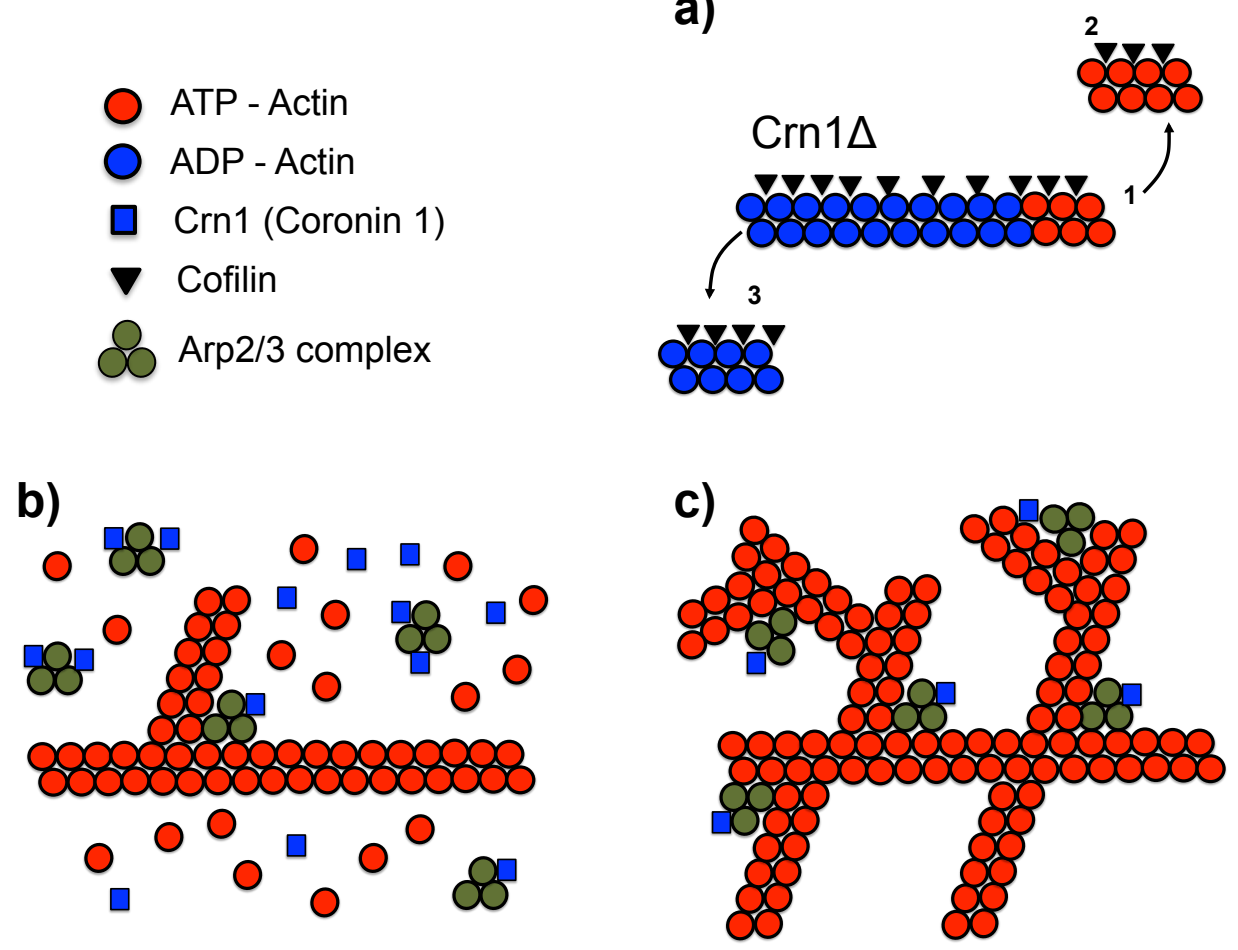

Figure 2.7: Visualization of a) the severing observed in the absence of Crn , and the F-actin branching process when b) Crn is above a critical concentration and c) when its below the critical concentration.

\subsubsection{Cooperative effects of Coronin 1A and Aip1}

There is evidence of cooperative effects between Coronin 1A and Aip1 in Dictyostelium discoideum regulating the dynamics of the actin cytoskeleton [50]. Re- 


\subsection{ACTIN CYTOSKELETON}

moval of these two proteins by genetical techniques results in cell migration velocities decreased 6-fold in the growth phase and 7-fold in the developmental phase. The cell colony growth rate decreases by $60 \%$ in a double knockout strain compared to the wild type and it takes 4 hours more to enter into the early developmental (chemotactic) phase. The cells also have an excess of filamentous actin and are multinucleated. These cells were also impaired in disassembling the filamentous actin after endocytosis which lasted 2 times longer than in wild type cells. These observations can be explained by observation of the cooperative effects between Cofilin, Coronin 1A and Aip1 in in vitro studies. These cooperative effects are actin bursting and shielding of capping proteins [59].

Both of these effects have been observed in in vitro assays that consisted of induced actin polymerization in perfusion chambers by flowing HeLa cell extract with Listeria monocytogenes adhered to the cover slips. Listeria monocytogenes is a parasitic bacteria that infects cells and creates actin comet tails to propel around [104]. As the extract flows through the chamber the Listeria will polymerize actin, and to depolymerize it the authors replaced the Hela solution with one having the depolymerization factors. In [14] the actin filaments were pre incubated with either cofilin, Aip1 or Coronin 1A and then a flow was started with the remaining depolymerization factors. If pre incubation was done with either cofilin or Aip1 the filaments were stable. Though if the filaments were pre incubated with Coronin 1A the filaments were unstable at the moment the rest of depolymerization factors were flown (Figure 4.11, left).

In [59] the authors using the same assay observed that the actin filaments would have sudden bursts with exponentially distributed random times. In the same fashion as in the previous experiment they studied the effects of pre incubation with CapZ, a protein that caps actin filaments with high affinity. If the actin filaments were pre incubated with depolymerization factors, the flow of CapZ would have low affinity to the actin filaments. The other way around when CapZ was used to pre incubate the actin filaments, the depolymerization factors would not recognize the actin filaments (Figure 4.11, left). These results can be interpreted as a mutual inhibition between the depolymerization factors and the capping proteins, this is similar to what was concluded in a study reviewed in a previous section [70]. 


\section{Actin burst}

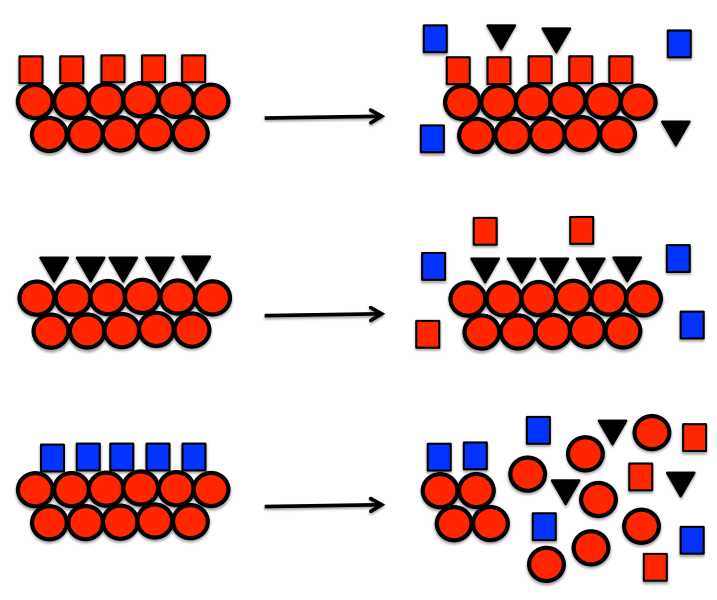

Shielding effect

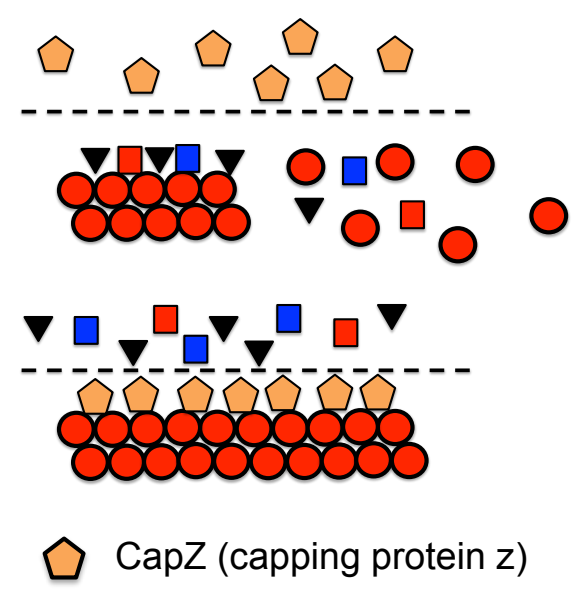

Figure 2.8: Cooperative effects of Coronin 1A and Aip1. Incubation with Coronin $1 \mathrm{~A}$ causes the actin filament to burst (left). Shielding depolymerization when actin filaments are incubated with capping proteins and shielding of capping proteins when incubated with depolymerization factors (right).

\subsection{Dynamical properties and time scales of the signaling cascade and actin cytoskeleton}

As a living organism, the biological processes inside a cell operate at different time scales in order to confront the survival demands imposed by external cues. In the case of Dictyostelium discoideum the dynamics of the biological processes have been investigated for several years. Although these processes are complex in nature, their study on the basis of nonlinear dynamics and pattern formation has been shown useful to understand them better [43, 102, 4, 121, 48]. The times scales involved in these phenomena ranges from fast time scales in the order of milliseconds to slower ones in the range of hours. A given time scale is not associated to an specific component of the actin cytoskeleton or signaling system, instead several time scales can be observed in the same component. Here we review the observed dynamical properties sorted by the time scales in which they appear. 


\subsection{DYNAMICAL PROPERTIES AND TIME SCALES OF THE SIGNALING CASCADE AND ACTIN CYTOSKELETON}

\subsubsection{Fast time scales, order $\sim 1 s$}

As discussed before the first step in the detection of cAMP is carried out by binding to the CAR. The dissociation rates of the cAMP receptors are in the order of $1 \mathrm{~s}^{-1}$ according to their phosphorylation state [106] and the association rate is $5.6 \mu M^{-1} s^{-1}[3]$. As we will review this is the fastest process for the signaling cascade and therefore there are three orders of magnitude in the difference between the time scales with the slowest time scale which is related to cAMP production.

\subsubsection{Intermediate time scales, order $\sim 10 \mathrm{~s}$}

The dynamics of the signaling network present variations in time scales of two order of magnitude between autonomous and induced responses. By changing the external background concentration of cAMP, Ras becomes transiently activated [100]. From response initiation to adaptation to a new cAMP background takes around $20 \mathrm{~s}[93$. The ability of the cell to adapt to external background changes has been investigated, on [61] it was proposed that the adaptation kinetics can be explained by an incoherent feedforward loop model which the authors named LEGI (Local excitation and global inhibition). The model relies in the existence of a hypothetical fast activation process that acts on the cell membrane while a slower diffusive inhibition process changes the sensitivity to the environment. This hypothesis was tested by comparing the responses of Ras to external doses of cAMP, it was concluded an incoherent feedforward model fits best the data than an integral feedback model (which also shows adaptation). From this study it was proposed that RasGEF acted as the fast activator and RasGAP as the slow inhibitor [101].

Under external cAMP stimulation the responses of PIP3 and PTEN have the same time scales as Ras $(\sim 20 s)$ [32]. As mentioned in a previous section it is known that the presence of PTEN is essential for a cell to guide towards the chemoattractant. In Etzrodt et al. [32] the cAMP responses of several components of the cytoskeleton were measured, such as Aip1 and Coronin 1A. It was shown that these proteins exhibit similar response time scales of around $20 \mathrm{~s}$. In this work we will concentrate in the time scales of the components of the actin cytoskeleton. Periodic forcing with pulses of cAMP revealed a resonance of around $20 s$ (a 


\subsection{DYNAMICAL PROPERTIES AND TIME SCALES OF THE SIGNALING CASCADE AND ACTIN CYTOSKELETON}

detailed account of these experiments will be given later) [114]. But also it has been observed by the author of this work and colleagues that the cytoskeleton also shows time scales of around $10 s$ with periodic polymerization/depolymerization cycles.

\subsubsection{Slow time scales, order $\sim 100 s$}

Developmental and genetical processes inside cells occur over the time scales of hours. For example, the expression of cAMP receptors can be induced in laboratory by withholding nutrients from the cell and pulsing external cAMP every six minutes for six hours [100]. After expressing CAR the cells will emit pulses of cAMP, the periodicity of the pulses changes over time and can range from $30 \mathrm{~min}$ to 6 min after 5 and 7 hours of nutrient deprivation respectively. In experiments using perfusion chamber it was found that the ratio between cell density and flow speed was a critical parameter for the initiation of cAMP pulsing [43].

The phosphatidylinositol (PIP2 and PIP3) system is the one that has its dynamics characterized better. In cells treated with Latrunculin A, which depolymerizes the cytoskeleton, it was observed that in a confocal slice of the cell membrane displays rotating PIP3 waves. The time series obtained from a single point display fast time scales associated with concentration changes and slow ones in which concentrations are nearly stable. This process has a periodicity of $T \sim 200 \mathrm{~s}$ and is reminiscent to relaxation oscillations [4]. Observations on the lower part of the cell membrane from another study have shown the existence of spiral waves, and it was claimed by the authors that the nucleation and collision of resulting phase singularities with the cell edges are correlated to morphological changes [102].

Motile cells exhibit patches of PIP3 that are related to pseudopod extension, with a typical lifetime of one minute [86]. The mechanism proposed for this observation has been an excitable system, in which the inhibiting variable diffuses faster resulting in small patches [46]. In [121] the authors performed numerical studies and concluded that coupling the LEGI model with an excitable system reproduces many of the observed features in cells. In steady state the cell membrane has a low PIP3 and a high PTEN concentration, occasionally the PTEN levels are depleted in small regions that travel around [40]. These domains termed holes where 


\subsection{OBJECTIVE}

modeled in [55] by coupling a bistable reaction diffusion system with an excitable system as a central pattern generator.

In [39] the dynamics of actin polymerization and signaling system were visualized. Cells were labelled with LimE and PTEN and monitored during their recovery from Latruculin A, it was shown that the observed actin and PTEN waves had timescales in the order of hundred of seconds. By using FRAP (fluorescence recovery after photobleaching) [62] it was concluded that these waves consisted of actin treadmilling instead of active transport by molecular motors [12]. A detailed analysis in the morphodynamics during gradient sensing has shown that the cell shape changes over time can be explained by two wave modes obtained by principal components analysis. The periodicity of these transitions are in the order of $200 s$ similar to the observed periodicities in the phosphatidylinositol system when treated with Lantruculin A. An understanding of the different timescales appearing in most components of the chemotactic machinery is far from understood, a proposal made to reconcile these ideas was of a slow signaling cascade is coupled to a fast cytoskeleton [48], though it would not explain the intermediate time responses of PTEN when a cell is stimulated. It is possible that this time scale distribution arise naturally in biochemical networks with a high number of degrees of freedom. Finally an important concept while analyzing data is the intrinsic cell to cell response variability, this will affect the statistical averaging since it implies that every cell has different internal parameters. In 92] it was shown that an statistical average of induced Ras responses by steps of cAMP had a high amount of variation between cells. Though repeated single responses in a single cell would have a low amount of variation between each other. Also it has been observed that from cell to cell there is a different threshold to induce a response [111]. These effects are taken into account while analyzing the data in this work and will be explained in the next chapter. This survey of time scales assists in understanding the observed phenomena in this current work.

\subsection{Objective}

In Westendorf et al. [114] we have studied the physiological responses of the actin cytoskeleton to external pulses of cAMP. It was shown that the average cytosolic 


\subsection{OBJECTIVE}

intensity, which corresponds to the amount of actin monomers, depletes and relaxes back to equilibrium showing an overshoot (Figure 2.9 a). The time series were reminiscent to the response of a damped oscillator. Further investigations with periodic stimulation have shown that it has a resonant frequency at $T \sim 20 \mathrm{~s}$, confirming its oscillatory properties. It was shown also that genetical knockouts, Aip1 $\Delta$ and $\operatorname{Crn} \Delta$, responded differently at this frequency, reflecting their specific role in the dynamics.

A remarkable observation is that in $\sim 10 \%$ of the observed cells exhibit self sustained polymerization cycles reminiscent to limit cycle oscillations (Figure 2.9 d). It was conjectured that this appears as a consequence of the actin cytoskeleton being operating near an oscillatory instability, and due to natural cell to cell variability, each cell would be at a certain distance from Hopf bifurcation. The detailed nature of these oscillations was not determined, for example they could arise by a purely deterministic mechanism, as they could also be purely stochastic in nature, being induced by the environmental noise [71]. As we are studying a biological system both deterministic and stochastic processes must be present. Fortunately there are several theoretical studies that guide us into looking for specific dynamical and statistical parameters that can be extracted from the data [60, 91]. Therefore one of the objectives of this work is to do a systematic comparison between different possible mechanisms and their predicted parameters, to eventually determine the mechanistic nature of these oscillations.

The second part of this thesis will be devoted to the following questions: How are the responses of actin polymerization affected in the absence of the proteins Aip1 and Coronin 1A? and what biochemical roles can be inferred by observing the biophysical processes? So in a similar manner as in [114] the cytoskeletal response to cAMP pulses will be studied by monitoring LimE and the proteins that regulate depolymerization, along with genetical knockouts of Aip1 or Coronin 1A. 


\subsection{OBJECTIVE}

A
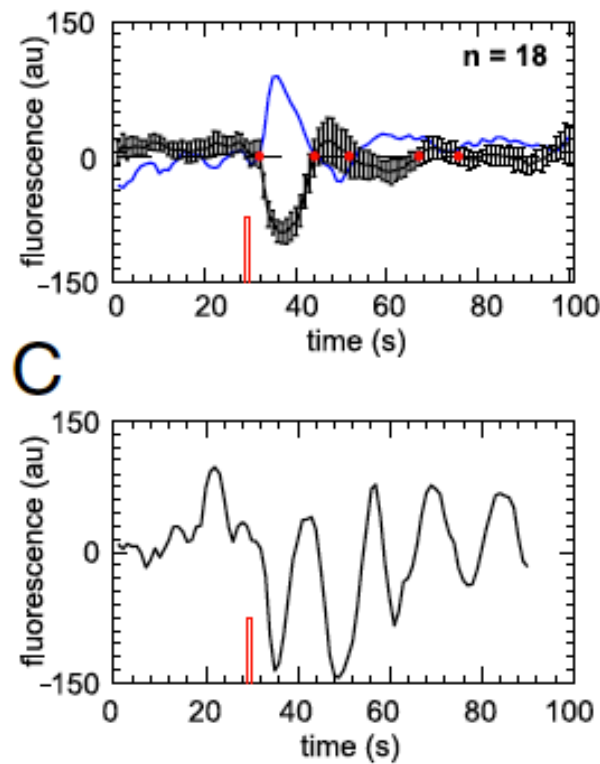

$\mathrm{B}$
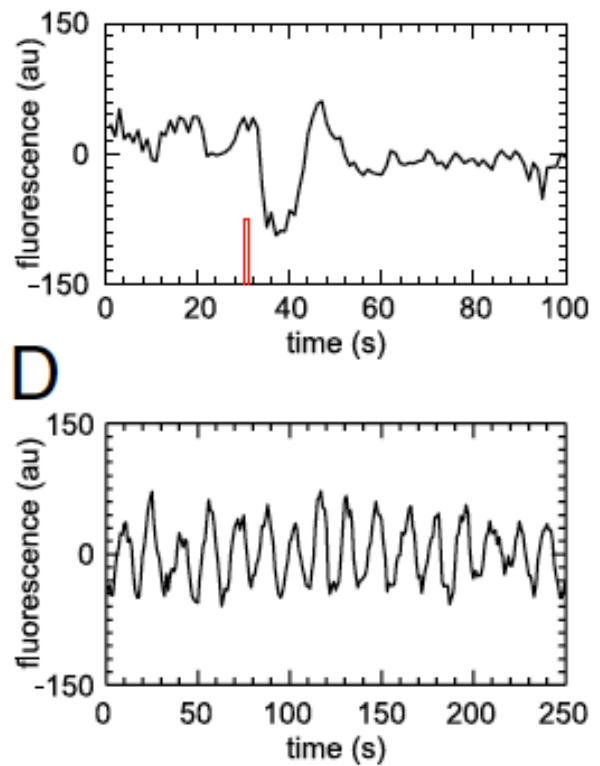

Figure 2.9: Average cytosolic fluorescence intensity for LimE [114] in response to a pulse of cAMP. The responses are reminiscent to damped oscillations: a) average time trace, b) cell with fast damping and c) with slow damping. While d) corresponds with self sustained activity reminiscent to limit cycle oscillations. 
2.5. OBJECTIVE 


\section{Chapter 3}

\section{Materials and methods}

In this section we will review the methods and tools used to perform our experiments and data analysis. As mentioned in Chapter 2 we are interested in studying cells during their chemotactic stage. Therefore it is essential to mention the procedures to store cell spores for long times, as also the starvation protocol to drive cell colonies from the vegetative to the developmental stage. These protocols have been used in different strains where either cells are coexpressing Aip-GFP or Coronin 1A-GFP with the filamentous marker LimE-mRFP, or in knockouts of Aip1 or Coronin 1A with LimE-GFP marked.

In the experiments single cells were stimulated with pulses of cAMP by means of flow photolysis [8]. Space will be devoted to review the advantages of flow photolysis and as well the precautions that need to be taken when using it. Since flow photolysis is performed in microfluidic devices, details of techniques used for soft lithography will be mentioned, as also details of the experimental set up and the parameters used during experimentation.

The details of how the imaging was performed will be mentioned, we visualized confocal images of fluorescent markers coming from single cells using a confocal laser scanning microscope (CLSM). The images obtained were segmented using the Chan-Vese algorithm to calculate the average cytosolic fluorescence. From these time series were obtained, the analysis used will be mentioned such as fluctuation analysis via the autocorrelation function. Finally we also mention the theoretical interpretations of the parameters obtained from the autocorrelation function, that 


\subsection{CELL CULTURE}

have been vital for the later interpretation of our data.

\subsection{Cell culture}

All the cells used in this work belong to the axenic cell line AX-2 [112]. An axenic cell line is one that has the ability to survive without their natural aliment. So instead of using bacteria, the cells are maintained in dishes with HL-5 medium (composition found in [112]) for their nourishment [33]. For long term storage spores are kept in a freezer at $-80^{\circ} \mathrm{C}$ and for usage they are thawed in a water bath at $37^{\circ} \mathrm{C}$. Then they are transferred to $10 \mathrm{ml}$ of HL-5 and are allowed to recover for 30-60 min at $21-23^{\circ} C$ [33]. These strains have a doubling time of 8-9 h with a saturation cell density at $4 \times 10^{6}$ cells $/ \mathrm{ml}$, and grow optimally at $21-23^{\circ} \mathrm{C}$. The cells used in this work had the following mutations: LimE-mRFP+ Coronin 1A-GFP, LimE-mRFP+Aip1-GFP, LimE-GFP+Aip1 $\Delta$ and LimE-GFP+Crn $\Delta$ (details of the constructs can be found in [34, 38, 56, 50]). For subculture the cells are kept in Petri dishes filled with $10 \mathrm{ml}$ of HL-5 at $22^{\circ} C$, the cells are splitted every 2-3 days for LimE mRFP+Crn-GFP and LimE-mRFP+Aip1-GFP, and every 1-2 weeks for LimE-GFP+Aip1 $\Delta$ and LimE-GFP $+\operatorname{Crn} \Delta$. After 11-13 passages the cells are discharged and a new stock is taken from the freezer.

The cell cultures were prepared for experiment one day in advance. A drop of cell solution with $1 \times 10^{6}$ cells (determined with the Neubauer chamber) is mixed with $25 \mathrm{ml}$ of HL5 medium in a flask. The flask is then put into shaking culture at $150 \mathrm{rpm}$ and the temperature maintained at $22^{\circ} C$. 7 hours before the experiment the cells are centrifuged at $1000 \mathrm{rpm}$ at $4^{\circ} C$ for 3 minutes. The supernatant is then removed and the cells are washed with $10 \mathrm{ml}$ of phosphate buffer $(\mathrm{pH}=6)$, this process is repeated twice. The remaining pellet is resuspended afterwards in $20 \mathrm{ml}$ of phosphate buffer and then transfer to a $50 \mathrm{ml}$ flask. The culture is shaken for 30 minutes in phosphate buffer, after that it receives two drops of $25 \mu l$ of cAMP (at $16.7 \mu \mathrm{M}$ ) every six minutes for six hours. This cAMP pulsing protocol is for stimulating the expression of CAR's in the cells [100]. After the six hours have passed, the cells are centrifuged again with the same parameters as before. The remaining pellet is suspended and mixed with $2 \mu \mathrm{l}$ of phosphate buffer and the cells are inserted into a microfluidic device for experiment. 


\subsection{FLOW PHOTOLYSIS}

\subsection{Flow photolysis}

As discussed in Chapter 2 the response to cAMP is in the order of seconds for several components of the actin cytoskeleton [32]. Therefore to understand the underlying dynamics of this system, we need to provide chemical stimuli that is quantitatively controlled at a subsecond resolution and at single cell scales. The experimental technique of flow photolysis was developed to address these issues [8]. The technique subjects the cells to a constant flow of caged compound in a straight microfluidic channel, Figure $3.1-1$, a. The caged compound consists of a chemical caged fused with the ligand of interest, cAMP in this case, and is released from its cage by exposing it to short wavelength radiation. The caged compound is biologically inert and therefore the cell cannot sense it before photoactivation. As shown in Figure $3.1-1$ (b) the caged compound can be release in front of the cell, with the possibility of exposing it to local gradients by controlling the irradiation region.

In using microfluidic devices for single cell stimulation, the effects of the interplay between solute diffusion and fluid flow must be taken into account. Geoffrey Taylor showed in 1953 that for a straight laminar flow in a pipe, diffusion is enhanced in the direction perpendicular to the flow. Later Aris [5] generalized this analysis for any tube geometry, and this effect is called the Taylor-Aris dispersion. In [6] the effects of the Taylor-Aris dispersion given by the uncaging switching time were studied for a straight microfluidic channel. The switching time was defined the time needed to increase the concentration of a photoactivated compound from $5 \%$ to $95 \%$ of maximal concentration. Figure 3.1 (2) shows their findings, it can be seen that there is a dependence for the switching time against the distance downstream from the source. The switching time monotonically increases the further is the target from the uncaging region, this implies that a single cAMP pulses becomes broader in the time domain as it travels through the device.

\subsection{Experimental set up}

The basic setup for this experiment consisted of four parts : The first is a straight channel microfluidic device which is filled with the solution containing the cells 


\subsection{EXPERIMENTAL SET UP}

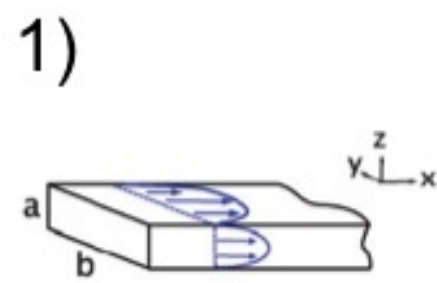

(a)

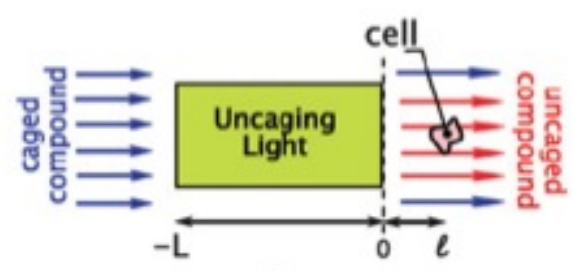

(b)

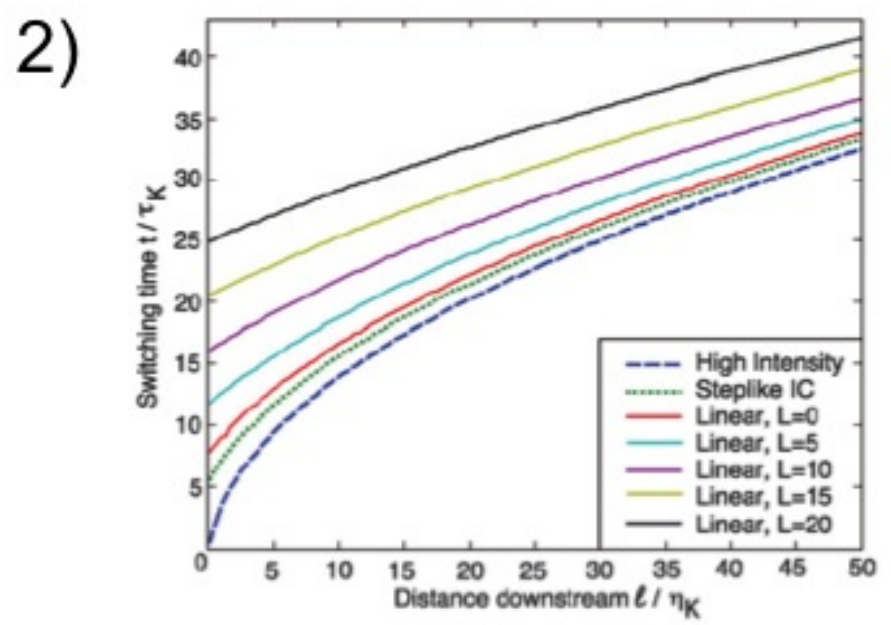

Figure 3.1: 1) Schematic of flow photolysis for cell stimulation, the uncaging area must be set in front of the cell. 2) The relation between switching time, which is the time needed to uncage from $5 \%$ to $95 \%$ of chemical concentration, and the distance from the uncaging region. The farther is the cell the longer it takes to get to the highest concentration, this is due to the Taylor-Aris dispersion. (Figure modified from Bae et al. [6]) 


\subsection{EXPERIMENTAL SET UP}

(Figure 3.2 a). Next in the list is the BCMCM caged cAMP which was used to stimulate the cells, this was flown into the microfluidic device by a $250 \mu l$ syringe (Hamilton, Bonaduz, Switzerland) mounted on a Standard Infuse Syringe Pump (Harvard Apparatus PHD 2000, Holliston, MA) (Figure 3.2 b) at constant speed. In order to visualize the cells and release cAMP from its cage, an Olympus FV-1000 confocal microscope was used (Figure $3.2 \mathrm{c}$ ).

Figure 3.2 shows the experimental set up used in every experiment, in the left picture it can be observed the syringe pump (Figure $3.2 \mathrm{~b}$ ). The pump contains a $400 \mu$ l Hamilton syringe (model 750) containing the BCMCM caged cAMP and attached to the syringe there is tubing connecting it with the microfluidic device. In the left picture of Figure 3.2 we can see the microfluidic device with its tubing (dimensions, $0.56 \times 1.07 \mathrm{~mm}$ ) connected to the syringe pump, the device is on the confocal microscope and below it is the objective to visualize the cells. In the next sections these components are explained in more detail.

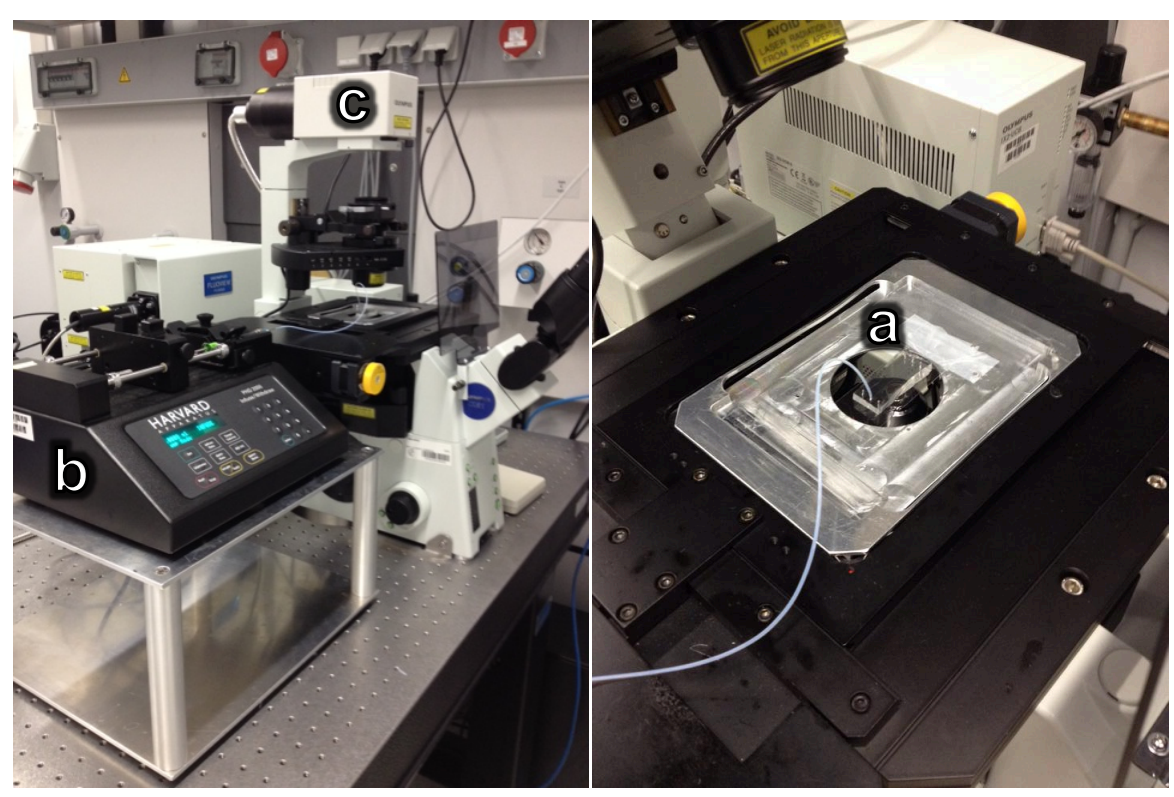

Figure 3.2: Experimental set up, a) Microfluidic device for cell stimulation, b) Standard Infuse syringe pump and c) Olympus FV-1000 confocal microscope. 


\subsection{EXPERIMENTAL SET UP}

\subsubsection{Soft lithography}

A sketch of the microfluidic device used in this work is shown in Figure 3.3, it consists of straight channels of $500 \mu \mathrm{m}$ in width, $30 \mathrm{~mm}$ long and with a height of $26 \mu \mathrm{m}$. The microfluidic devices were created from the master wafer by covering it with a mixture of $60 \mathrm{~g}$ of polydimethylsiloxane (PDMS) (Sylgard 184 Silicone Elastometer, Dow Corning) and $6 \mathrm{~g}$ of its curing agent (Sylgard 184 Silicone Elastometer Curing Agent, Dow Corning). The master wafer with the mixture was brought into an dessicator for degasing and then it was cured for two hours at $75^{\circ} \mathrm{C}$.

Single devices were cutted out with a scalpel and cleaned with magic tape several times. Then the inlets/outlets were punched with a 19 gauge stainless steel syringe tip. The cover slip was also cleaned with magic tape and then brought into a plasma cleaner (Harrick Plasma Cleaner, Ithaca, NY) along with the device. The device was held at the plasma cleaner for 1.5 minutes, in the first minute the vacuum was set and in the remaining time the radio frequency radiation ( $R F)$ was turned on at the highest level to create the plasma. After plasma cleaning the coverslip and PDMS were adhered to each other and brought into the oven at $75^{\circ} \mathrm{C}$ for one minute.

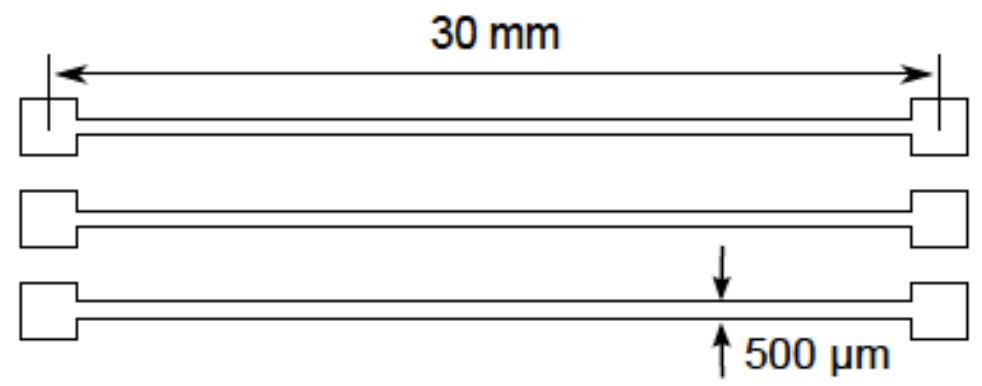

Figure 3.3: Design of microfluidic device used in flow photolysis experiments. (taken from Westendorf et al. 2012 [113]) 


\subsection{EXPERIMENTAL SET UP}

\subsubsection{Caged compounds}

In all the experiments the caged compound used was BCMCM-cAMP (6, 7Bis(carboxymethoxy)coumarin- 4- yl)methyl- adenosine- 3', 5'- cyclic monophosphate) (Biolog Life Sciences Institute, Bremen, Germany). The BCMCM powder was dissolved in phosphate buffer and diluted to $10 \mu M$. The solution was kept in $500 \mu \mathrm{l}$ aliquots at $-75{ }^{\circ} \mathrm{C}$ until thawing prior to the experiment. Then it was inserted into a $500 \mu l$ syringe (Hamilton, Bonaduz, Switzerland) covered in aluminum foil.

\subsubsection{Confocal microscopy}

The experiments were performed using the confocal laser scanning microscope Olympus FV1000. For all the experiments the microscope had an UPLSAPO $60 X O$ objective with oil immersion. A multiline $30 \mathrm{~mW}$ Argon laser (Melles Griot, Carlsbad, Ca) was used to excite the GFP fusioned with the proteins, for this the $488 \mathrm{~nm}$ laser line was used at 10\% of maximum intensity for each cell. The fusioned mRFP was excited by using $1 \mathrm{~mW}$ HeNe laser (Melles Griot, Carlsbad, Ca) with a wavelength of $543 \mathrm{~nm}$, for each experiment the laser intensity was set to $20 \%$ of maximum intensity. The photoactivation of caged compounds was done with a laser diode with a wavelength of $405 \mathrm{~nm}$, for each experiment the laser was used at full power of $25 \mathrm{~mW}$. The image detection was performed in separate channels when two fluorophores were present and with each image scanned sequentially, the voltages of the photomultipliers were varied from $400-600 \mathrm{mV}$ to maximize the dynamic range. The images were scanned sequentially in order to avoid the artifacts of spectral bleeding (which happens when the spectra of two fluorophores have an overlap and can be detected by both channels). As shown in Figure 3.4, a region of $48 \times 48 \mu \mathrm{m}(120 \times 120$ pixels $)$ was determined for imaging, on the most left side another region of $0.4 \times 48 \mu \mathrm{m}(1 \times 120$ pixels $)$ was determined for the uncaging of cAMP by the scanning laser (405 nm; $25 \mathrm{~mW}$ ). Each cell was imaged at a height $\sim 2 \mu \mathrm{m}$ from the bottom of the coverslip, and the cell was centered as much as possible to keep it $\sim 20 \mu \mathrm{m}$ from the uncaging region. The flow profile streamed perpendicular from the uncaging region from left to right of the imaging region. The images for both green and red lasers were scanned at a rate $4 \mu \mathrm{s} /$ pixel 


\subsection{SIGNAL PROCESSING}

taking $0.48 \mathrm{~ms}$ per image, we used a frame rate of one second to scan between images of the same fluorophore. A rate of $2 \mu \mathrm{s} /$ pixel was used for the LD laser and took $0.24 \mathrm{~ms}$ for a single scan, in each experiment the cAMP administered to the cell lasted $1 s$ and in that time the uncaging region was scanned $\sim 4166$ times.
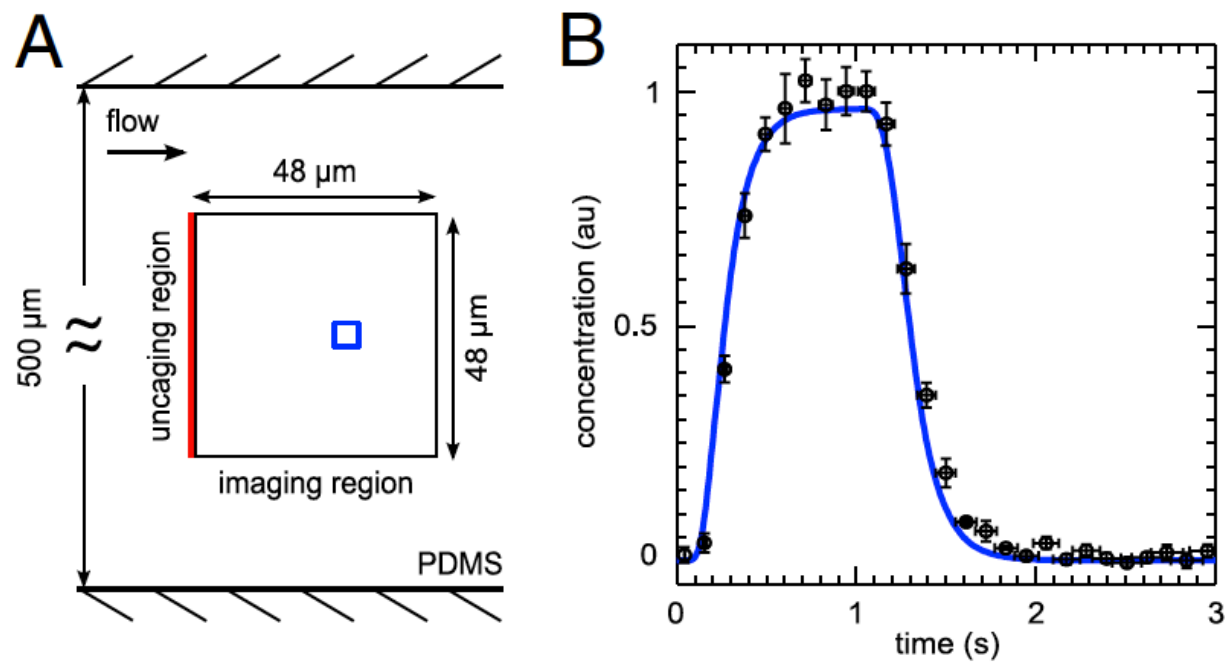

Figure 3.4: A) Schematic of the microfluidic device along with the $48 \times 48 \mu \mathrm{m}$ imaging region, the uncaging region is denoted by a red line. B) Time series of the uncaging profile measured with fluorescein at the pixel denoted by a blue square in A. (Taken from Westendorf et al. 2013 [114])

\subsection{Signal processing}

\subsubsection{Data retrieval}

To analyze the images recorded from the confocal microscope, the routines written by Christian Westendorf were used [113]. The first step to retrieve the data is to segment the image for extracting cell contours. As the cell is imaged continuously, its fluorophores photobleaches [105] and the mean fluorescence intensity decreases over time. Therefore if a boundary is found by segmenting an image with a fixed 


\subsection{SIGNAL PROCESSING}

fluorescence threshold, the boundary would be subject to photo bleaching giving deceiving results. An adaptive algorithm is needed then, and in this work we have used the Chan-Vese algorithm for image segmentation [19].

The Chan-Vese algorithm uses a Free energy functional of the form

$$
\begin{aligned}
F\left(c_{1}, c_{2}, C\right)= & \mu \text { Length }(C)+\nu \text { Area }(\text { inside }(C)) \\
& +\lambda_{1} \int_{\text {inside }(C)}\left|u_{0}(x, y)-c_{1}\right|^{2} \mathrm{~d} x \mathrm{~d} y \\
& +\lambda_{2} \int_{\text {outside }(C)}\left|u_{0}(x, y)-c_{2}\right|^{2} \mathrm{~d} x \mathrm{~d} y
\end{aligned}
$$

where $\mathrm{C}$ is an involving curve that evolves over time to the image objects boundaries, $u_{0}(x, y)$ is the image, $c_{1}$ and $c_{2}$ are the averages of $u_{0}(x, y)$ inside and outside the curve $\mathrm{C}$ respectively, $\mu \geq 0, \nu \geq 0, \lambda_{1}>0$ and $\lambda_{2}>0$ are constant parameters. The time evolution of curve $\mathrm{C}$ is defined by

$$
\inf _{c_{1}, c_{2}, C} F\left(c_{1}, c_{2}, C\right)
$$

Figure 3.5 shows an example of image segmentation performed to an image with a single cell. An initial curve $\mathrm{C}$ circling the whole imaging region, will evolve to the boundary of the cell denoted by the blue curve. We have used the parameters $\mu=0.25, \nu=0, \lambda_{1}=-1$ and $\lambda_{2}=1$ which are the same used by the authors of the Chan-Vese algorithm in the original paper [19].

Once the cell is detected two regions are determined: the cell cortex is a band surrounding the cell that goes from the cell boundary to $1.6 \mu \mathrm{m}$ inside the cell, and the rest was defined as the cytosol. To quantify the dynamics of both regions over time, their mean spatial fluorescence intensity over time was calculated

$$
I(t)_{c y t, c o r}^{*}=\overline{I(t)_{c y t, c o r}}-\overline{I(t)_{B g n d}}
$$

where $\overline{I(t)_{\text {cyt,cor }}}$ is the mean fluorescence averaged over the cytosol, and $\overline{I(t)_{B g n d}}$ is the mean background fluorescence. As mentioned in the background section, there is a cell to cell variability due to their different internal physical parameters. 


\subsection{SIGNAL PROCESSING}

In the next subsection we describe how the time series $I(t)_{c y t, c o r}^{*}$ were analyzed for single cells.

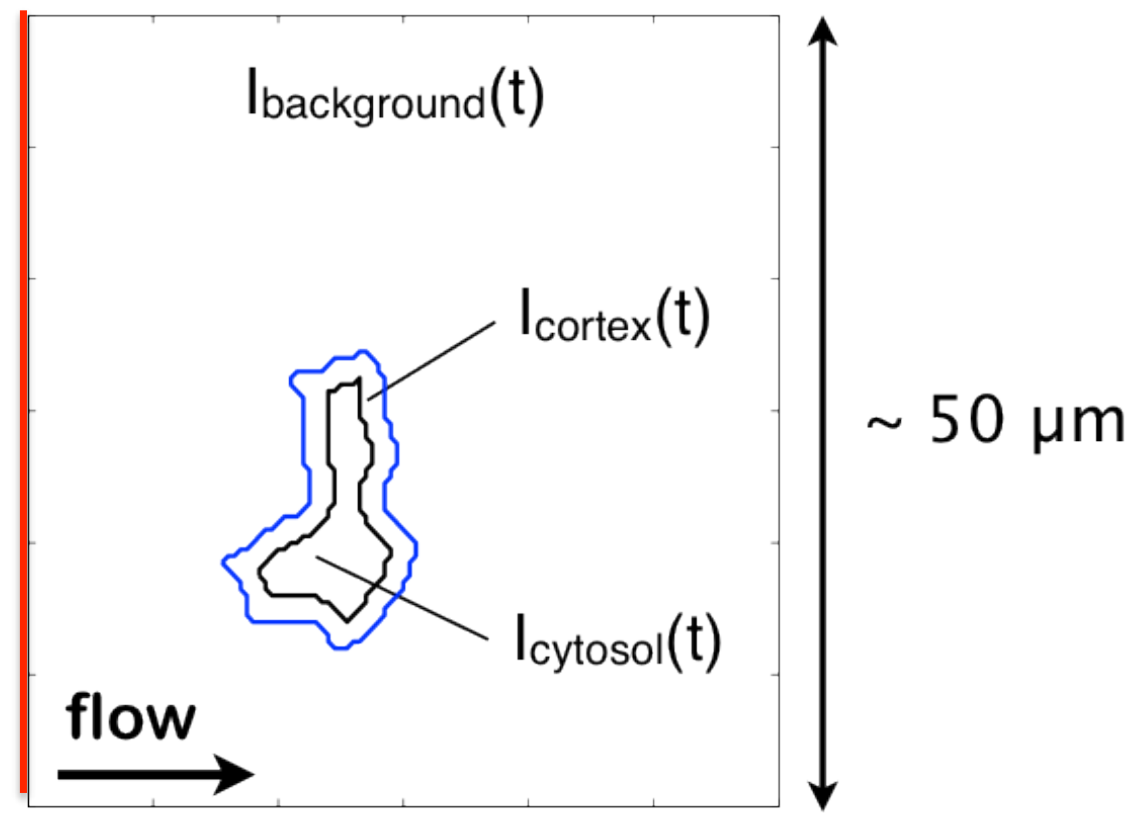

Figure 3.5: The Chan-Vese algorithm detects the cell contour to extract fluorescence intensity. We have defined the cortex as the region covering four pixels from the contour and the rest as the cytosol. The red line depicts the cAMP uncaging region. (Image from Christian Westendorf used with permission)

\subsubsection{Correlation analysis}

As mentioned in the previous chapter, to discern between different physical mechanisms we need to manipulate its internal physical parameters or give external perturbations. Unfortunately in biological experiments we might not have access to one or neither of them, so we complement the analysis by extracting physically meaningful parameters from the observed data. In this work we have relied substantially in techniques traditionally used in statistical mechanics [58], as the autocorrelation function 


\subsection{SIGNAL PROCESSING}

$$
C_{x x}(\tau)=\left\langle x(t-\tau) x^{*}(t)\right\rangle
$$

It gives the statistical features between different points of a signal, as well the time scales behind the physical process [15]. At $\tau=0$ the autocorrelation function gives the variance $\sigma^{2}$ of the time series. The decay rate of the autocorrelation function is given by the correlation time [97]

$$
\tau_{c}=\frac{1}{C_{x x}(0)} \int_{0}^{\infty}\left|C_{x x}(\tau)\right| \mathrm{d} \tau
$$

which is a measure of how fast several points decorrelate in a time series. The next tool is a measure of the relation between different points between two different signals called the cross correlation function

$$
C_{x y}(\tau)=\frac{\langle x(t-\tau) y(t)\rangle}{\sqrt{C_{x x}(0) C_{y y}(0)}}
$$

similar to the autocorrelation function except that is normalized. A value of one in the cross correlation means completely correlated and a value of 0 completely decorrelated.

If the Fourier transform of a signal is defined as $\mathcal{F}[x(t)]=X(\omega)$, then the power spectral density $\left(|X(\omega)|^{2}\right)$ and autocorrelation function are related via the Wiener-Khinchin theorem [15], expressed as

$$
C_{x x}(\tau)=\int_{-\infty}^{\infty}|X(\omega)|^{2} e^{-i \omega \tau} \mathrm{d} \omega
$$

These tools can be readily used to characterize the data, but also there is a theoretical framework for its interpretation, we proceed with a small review in terms of Langevin dynamics.

\section{Interpretation}

The tools just mentioned are useful by themselves, but it helps to give a physical interpretation of the parameters obtained. Here we review some of the interpretations that are given in the framework of linear dynamics [58], and as well in the framework of nonlinear oscillators [60, 91]. We start with the linear case, suppose 


\subsection{SIGNAL PROCESSING}

a physical system that can be defined by the following set of ordinary differential equations

$$
\frac{d}{d t} \vec{x}(t)=\mathbf{A} \vec{x}+\vec{\xi}(t)
$$

where $\vec{x}(t) \in \mathbb{R}^{n}, \vec{\xi}(t) \in \mathbb{R}^{n}$ and $\mathbf{A} \in \mathbb{R}^{n} \times \mathbb{R}^{n}$. The elements of the vector $\vec{\xi}(t)$ are defined as $\left\langle\xi_{i}\left(t_{1}\right) \xi_{j}\left(t_{2}\right)\right\rangle=D \delta_{i j} \delta\left(t_{1}-t_{2}\right)$, where $\mathrm{D}$ is the noise intensity, $\delta_{i j}$ is the Kronecker delta and $\delta\left(t_{1}-t_{2}\right)$ is the Dirac delta. The physical meaning of Equation 3.8 belongs to a system that is constantly forced with internal or external noise, for example: a passive particle in a fluid forced by the environment (Langevin Equation) [58], or as modelled in [68], an active cochlea hair bundle with internal and external noise.

Any system defined by Equation 3.8 cannot be directly solved analytically, but its statistical features can be obtained. By Fourier transforming Equation 3.8 we arrive at

$$
\vec{X}(\omega)=\mathbf{B} \vec{\Xi}(\omega)
$$

where $\mathcal{F}[\vec{\xi}(t)]=\vec{\Xi}(\omega)$ is the Fourier transform of the external noise vector, and

$$
\mathbf{B}=\left(i \omega I_{n}-\mathbf{A}\right)^{-1}
$$

where $I_{n}$ is the identity matrix. Finally the expression for the power spectral density (PSD) is given by

$$
\left|X_{i}(\omega)\right|^{2}=\left|\sum_{j=1}^{N} B_{i j} D_{i}\right|^{2}
$$

this quantity can be easily measured from the time series from an experiment. The following information can be obtained from the power spectral density: the number of major slopes in the curve gives number of components $n$ of the system. For example when $\vec{x}(t) \in \mathbb{R}^{1}$, the PSD will contain one major decaying slope only, as in the well known example of the Ornstein-Uhlenbeck process.

For the explicit case of a damped harmonic oscillator 


\subsection{SIGNAL PROCESSING}

$$
\begin{gathered}
\mathbf{A}=\left(\begin{array}{cc}
-\lambda & -\omega_{o} \\
\omega_{o} & -\lambda
\end{array}\right) \\
\vec{\xi}(t)=\left(\begin{array}{l}
\xi_{1}(t) \\
\xi_{2}(t)
\end{array}\right) \sqrt{D}
\end{gathered}
$$

we get the following PSD

$$
\left|X_{i}(\omega)\right|^{2}=\frac{D}{\lambda^{2}+\left(\omega-\omega_{o}\right)^{2}}
$$

and with the Wiener-Khinchin theorem (Equation 3.7) we get

$$
C_{x x}(\tau)=\frac{D}{2 \lambda} e^{-\lambda \tau} e^{i \omega_{o} \tau}
$$

Notice that the two important parameters mentioned before characterizes the dynamics of the oscillator, the variance $\sigma^{2}=\frac{D}{2 \lambda}$ is given by the balance between noise strength and dissipation rate, and the correlation time $\tau_{c}=\frac{1}{\lambda}$, is given by the dissipation rate of the oscillator. Another measure for the fidelity of the signal is given by the bandwidth of the PSD called the full width at half maxima (FWHM) which is proportional to the correlation time $\Delta \omega \sim \frac{1}{\tau_{c}}$.

For nonlinear systems there is no general expressions for the PSD, though in the case of analyzing a self sustained oscillator, several features observed in the PSD and autocorrelation function can also be linked to the dynamical properties of the system. For example the variance of the signal will also reflect the interplay between noise strength and energy input/dissipation rate $\sigma^{2}(\lambda, D)$ [91]. On the other hand, the correlation time is not easily defined for all parameters of a self sustained oscillator. In the case of large amplitude oscillations, we can approximate the amplitude by the variance $a(t) \approx \sigma^{2}[60$. In this case the signal decorrelates by the oscillators phase fluctuations called phase drift, these are influenced by the amplitude, and therefore the correlation time also depends on the variance $\tau_{c}\left(\sigma^{2}\right)$. Notice that even without explicit expressions, these parameters reflect the same properties as in the linear case. Since biological systems are noisy by nature [31, we will use these parameters to characterize the autonomous dynamics of the actin cytoskeleton of $D$. discoideum. 
3.4. SIGNAL PROCESSING 


\section{Chapter 4}

\section{Autonomous activity of the actin cytoskeleton}

As mentioned in the background section, a percentage of Dictyostelium discoideum cells exhibit periodic activity in actin polymerization [114]. The objective of this section is to gain some understanding on the mechanism leading to these oscillations. Several difficulties makes this task hard. First, the presence of experimental noise hinders the extraction of parameters. Also systems with fundamentally different dynamics may show similar characteristics. For example the behavior of a bistable system in the presence of noise [35, may be very similar to a system exhibiting relaxation oscillations [98], in the sense that the dynamics in both cases involves fast and slow time scales. More generally, distinguishing features attributable to the deterministic part of the dynamics, and to stochastic aspects in the system is the challenge we are addressing. Thus if a system is exhibiting both fast and slow times scales and periodicity, its mechanism cannot be identified without further tests. In this chapter, we used two main ways to distinguish between stochastic and deterministic aspects: one is the natural variation of internal parameters that an isogenic cell line will present [92, 111] and the second is giving external cAMP perturbations to analyze its response.

The first recorded instance of Dictyostelium discoideum cells with self sustained actin oscillations came from the double knockout Scar $\Delta / \operatorname{PIR} 121 \Delta$ [51], this suggested that an imbalance in the activators of the ARP2/3 complex was respon- 
sible for the transition between oscillatory and non-oscillatory states. An ideal experiment to test this hypothesis would consist in varying systematically the expression levels of SCAR and PIR, but the current genetical tools in Dictyostelium discoideum are limited to test it directly. Therefore our approach consists in perturbing the basal state of the actin cytoskeleton by applying external pulses of cAMP, and from its response infer some of their dynamical properties. As the control parameters for manipulating actin oscillations are unknown, we exploit the fact that an isogenic cell line exhibits a natural cell to cell variability in their biophysical properties [92, 111]. This reflects the internal distribution of control parameters that manipulate these oscillations, and as a consequence a percentage of cells are in the oscillatory state. At the same time other cells will exhibit the characteristic fluctuations induced by the environment, these are highly amplified when a dynamical system is close to a transition point between oscillatory and nonoscillatory states [116, 117]. We have exploited all these facts to give a complete characterization of the oscillatory actin cytoskeleton in chemotactic amoeba.

To identify the dynamical states in which the cells are, we used tools that are often used in statistical physics. The measured time series are characterized by the parameters given by the autocorrelation function (which contains its statistical features [15]). This is not the first time that these tools are used to analyze the dynamics of living cells, auto and cross correlation functions were used to discern the connectivity between different genes in E.coli. For example, the authors of a study measured the fluctuating expression levels of different proteins and they were able to relate the observed cross correlation with the pairwise interaction between genes [28]. In another case it has been used in eukaryotic cells to analyze the dynamics of the p53 DNA repair system. The authors fitted measured power spectral densities of the system components with solutions from Langevin equations. It was concluded that the interaction between different components could be tracked by this approach [41].

As mentioned in section 3.4.2, we interpret our measured autocorrelation functions using the seminal work of Melvin Lax (1967) [60] and the Fokker-Planck formalism for oscillators [91] as a guide. In these works the basis are laid to calculate the parameters that characterizes the autocorrelation function of a self sustained oscillator forced with additive noise. An important observation from these works 


\subsection{EXPERIMENTAL PROTOCOL AND MEASURED PARAMETERS}

is that the correlation time, which measures the decay rate of the autocorrelation function, can either be related to the oscillators amplitude or phase fluctuations (also known as phase drift). The amplitude fluctuations will only contribute to the background of the power spectral density, and therefore affects the autocorrelation function in its variance. The development of these techniques to calculate these parameters proven to be vital for the interpretation of our results.

\subsection{Experimental protocol and measured param- eters}

The cells used in this experiment coexpressed two fluorescent labels, either Coronin 1A-GFP+LimE-mRFP or Aip1-GFP+LimE-mRFP. To capture the cells in their different dynamical states they were chosen randomly in the microfluidic device for stimulation. Each cell was recorded either $400 s$ or $200 s$ by imaging sequentially both labels (the protocol was changed from $200 s$ to $400 s$ when a preliminary analysis had shown that it was more appropriate to record for longer times). After the first $100 s$ of recording, the cells were stimulated with a pulse of uncaged BCMCM cAMP, at a concentration of $10 \mu M$, for one second, and with the bleaching laser set to maximum intensity. The time series for average cytosolic fluorescence intensity were extracted for LimE and its companion label (Figure 4.2 a), an example of a cell expressing Coronin 1A-GFP+LimE-mRFP is shown in Figure 4.1. After the extraction of the time series the following steps were done.

First, the average value of the time series for the first $100 s$ was calculated and subtracted from the data. As the measurement progresses the fluorescence intensity will drift by photobleaching [62] or by a change in focal plane as the cell moves. Then, to detrend the slow time scales associated with these artifacts, a third order polynomial was fitted by using the least squares minimization method, and then it was subtracted from the data. Finally the fluorescence expression level also varies from cell to cell and the data needs to be scaled. The data was normalized based on the actin response induced by external cAMP stimulation, a parabola was fitted to the observed minimum by using the preceding and succeeding points. With the obtained algebraic expression for the parabola the minimum was found, and the 


\subsection{ACTIN BINDING PROTEINS AND PSEUDOPOD EXTENSION}

time series were divided by the absolute value of this minimum.

The dynamical state of the cell is not readily recognizable during the measurement, therefore the following steps were done to recognize it. The autocorrelation function (Eq. 3.4) was calculated for cytosolic LimE (Figure 4.2 b, red line), and for measurements in which the autocorrelation presented at least one oscillation cycle, it was classified as an oscillating cell. In total we have found 31 out of 119 cells to be classified as oscillatory. To extract parameters from the autocorrelation function the following formula was fitted (Figure $4.2 \mathrm{~b}$, dashed black line)

$$
C_{x x}(\tau)=\sigma_{\operatorname{Exp}}^{2} \exp \left[-\frac{|\tau|}{\tau_{c}}\right] \cos \left(\omega_{0} \tau\right)
$$

From the fit the parameters correlation time $\tau_{c}$ and variance $\sigma_{E x p}^{2}$ were obtained to characterize the cells. The autocorrelation function was fitted with a commercial software package ( MATLAB R2012b, The MathWorks Inc., Natick, MA, 2012 ) using the Levenberg-Marquardt algorithm for nonlinear least squares fitting (NLSF). A NLSF algorithm needs a parameter estimation as an initial condition, for the correlation time it was obtained by using Equation (3.5), to estimate the variance we used the value of the autocorrelation function at zero lag $C_{x x}(0)$, and for estimating $\omega_{o}$, we used the frequency of the main peak in the power spectral density. The cross correlation between LimE and the secondary label was also calculated with Eq. 3.6, an example is shown in Figure 4.2 c.

\subsection{Actin binding proteins and pseudopod ex- tension}

An important issue to address for the study of cell motility, is the relationship between cytosolic fluorescence depletion of actin binding proteins and pseudopod extension. The control of pseudopod extension is the direct link known so far in the interaction between the signaling cascade and the actin cytoskeleton. This link has been shown for example by depriving cells from the gene that encodes PTEN, which results in a significant impairment on the directionality of pseudopods [49]. The question per se needs a detailed study correlating the spatiotemporal dynamics 


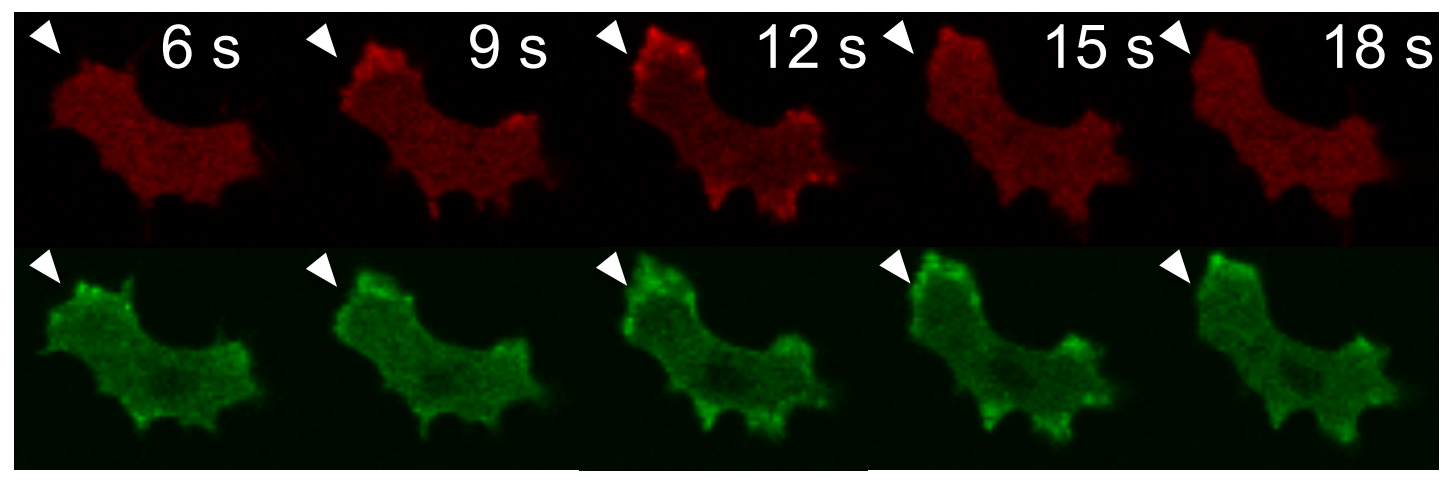

Figure 4.1: Example of a cell exhibiting periodic polymerization episodes in the actin cytoskeleton, the cell is labeled with green for Coronin 1A and red for LimE. The picture sequence corresponds to one period of oscillation. A region where a pseudopod was formed is marked by a white arrow, as the cytosol gets darker the pseudopod is extended even though after the cytosol recovers the pseudopod maintains its position.

of the cell boundary (with parameters such as curvature) and the mean cytosolic fluorescence signal, which is outside the scope of this work. As a first step to gain insight into this relation, we have taken the best measurement available and study the relation between pseudopod extension and the time series of cytosolic fluorescence intensity.

As mentioned in the materials and methods, we tracked the cell boundary with the Chan-Vese algorithm over time. In Figure 4.3 (right) we see the time evolution of the cell contour during one period of LimE oscillation. Each boundary is color coded for the observation time, which was defined such that $t=0 \mathrm{~s}$ corresponds to the minimum value of fluorescence in the given period. The contours were taken for the times $-6 s \leq t \leq 6 s$ with respect to the fluorescence minimum value and the coordinate system is given with respect to the center of mass of the cell at the frame $t=-6 s$.

Figure 4.3 (left) summarizes how the time evolution of the contours was quantified. For every oscillation period, the regions where the cell membrane protruded or retracted were identified. A straight line was drawn along a small region of the cell membrane (dashed black line in Figure 4.3 left), and normal to it an arrow was 

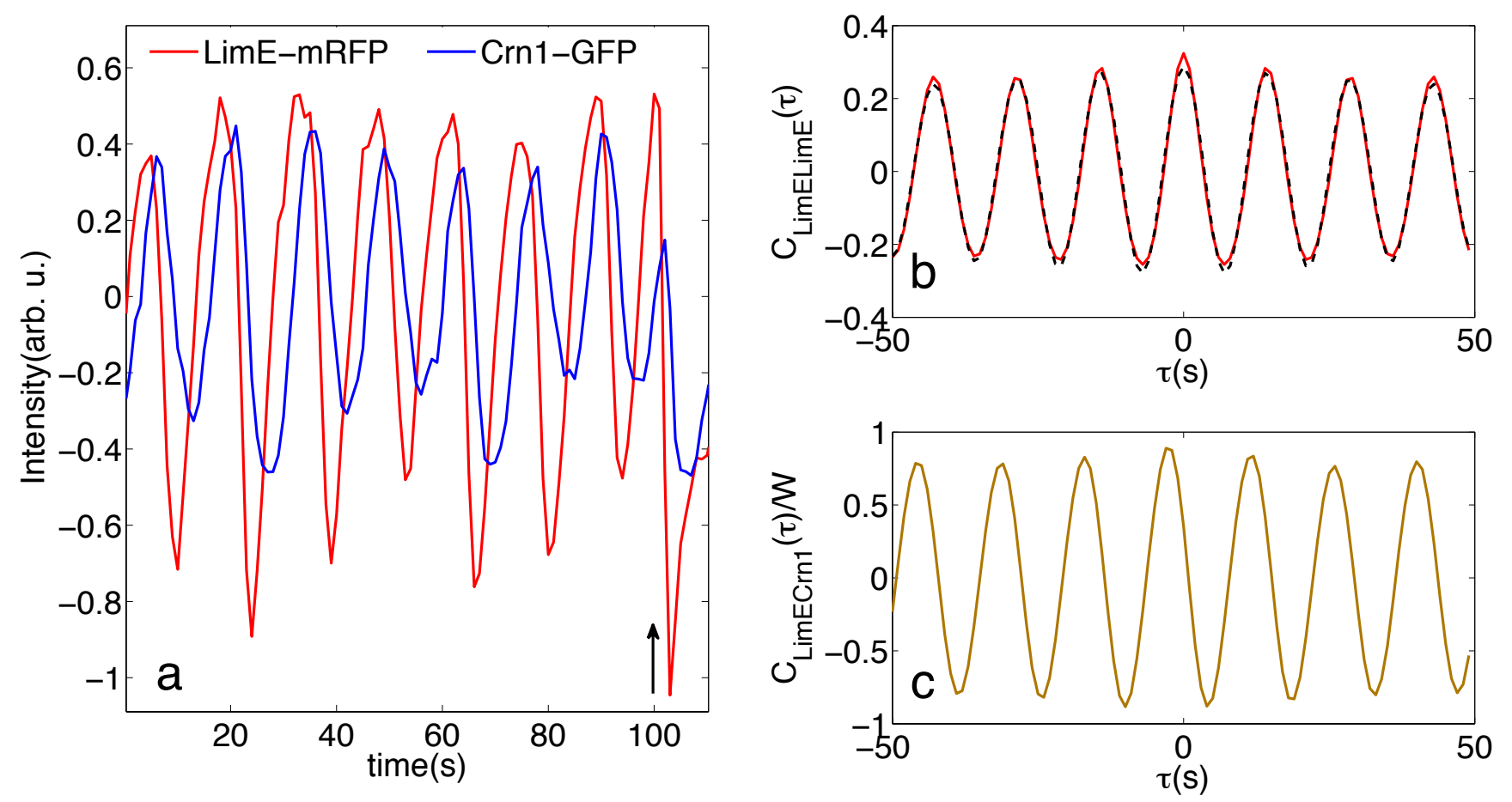

Figure 4.2: Data extracted from the cell shown in Figure 4.1. a) Extracted time series for mean cytosolic fluorescence for LimE (red) and Coronin 1A (blue), the black arrow corresponds to the time when the cell is stimulated with cAMP. b) The autocorrelation function from the LimE time series (red), the function of Equation (4.1) was fitted (black dashed line). c) Cross correlation function between LimE and Coronin $1 \mathrm{~A}$. 


\subsection{ACTIN BINDING PROTEINS AND PSEUDOPOD EXTENSION}

drawn pointing to the region the membrane evolves. In this example, an arrow was drawn normal to the cell membrane and is pointing outwards meaning that the cell membrane protruded. But also there are cases where the cell membrane retracted, meaning that the boundary would evolve inwards with respect to the cell center of mass (Example in Appendix D). Also there are cases where at the beginning protrudes and then retracts, the boundary in this case first evolved outwards and then inwards. The positions of the cell membrane touching the arrow were extracted and the time evolution for the length of the protrusion (retraction) were obtained by

$$
L(t)=\sqrt{\left(x(t)-x_{o}\right)^{2}+\left(y(t)-y_{o}\right)^{2}}
$$

where $x_{o}$ and $y_{o}$ are the position of the cell membrane at $t=-6 \mathrm{~s}$.

Our main finding from this analysis is summarized in Figure 4.4, on the left side of the figure we have an overlap of all the depletion periods with their corresponding minima centered at $t=0 \mathrm{~s}$. On the right side of the figure we have all the observed protrusion lengths for the corresponding time series. For each oscillation period, one or several pseudopods were created, so each line in Figure 4.4 (right) corresponds to a single protrusion. We can see that the times of pseudopod extension are well defined in the period $-4 s \leq t \leq-3 s$ before reaching the cytosolic fluorescence minima. Intuitively, we would have expected that LimE depletion at the cytosol would be correlated with pseudopod extension while fluorescence recovery with pseudopod retraction. We observed that the pseudopod length saturates and remains at that maximum point for a long time, while LimE fluorescence at the cytosol is recovered with no observed pseudopod retraction. The times to reach length saturation varied between pseudopods, but within the first two seconds of initiation reaches its maxima. This is fast compared to the LimE oscillation period which in this case was $T=14.28 \mathrm{~s}$. This suggest a relation between pseudopod length and the fluorescence signal $x(t)$ of the form

$$
L(t) \sim \int_{0}^{t} f(x(s)) \mathrm{d} s
$$

Meaning that cytosolic LimE fluorescence depletion represents the transient force exerted on the pseudopod (or cell membrane). 


\subsection{PERIOD AND PHASE DISTRIBUTION}

The dynamics of membrane retraction seem to be uncorrelated (Figure 4.5. right) with the fluorescence values. The retraction seems to be happening continuously with no actual defined timing for starting or finishing. Looking at Figure 4.5 (right), we see that the length of membrane retraction increases continuously in steps. Retraction is related to the dynamics of the motor protein MyosinII [21]. It is possible that MyosinII is exerting its force continuously opposite to actin polymerization. This observation supports the hypothesis in which the observed oscillations reflects the treadmilling of actin filaments and not an active translocations of filaments caused by molecular motors. Figure 4.5 (left) shows cases of apparent competition between protrusion and retraction, as it can be seen that the length of a pseudopod increases, but instead of reaching length saturation it decreases at later times. To the best of the author's knowledge the results presented here are new. These might help to understand the more general case when the polymerization dynamics are not periodic.

\subsection{Period and phase distribution}

From the parameters available, we proceeded to characterize two basic biophysical properties: period distribution and the phase lag between different proteins. The phase lag is defined as the phase angle where the cross correlation function has its maximum. This can be calculated by using $\Delta \phi=\omega_{o} \tau_{\max }$, where $\tau_{\max }$ is the time lag where the cross correlation is the highest, and $\omega_{0}$ is the extracted frequency from the LimE autocorrelation function.

First, with the extracted frequencies we proceed to analyze the oscillation period distribution. Figure 4.6 shows the distribution for the LimE time series. It can be observed that the majority of the cells $(n=17)$ have an oscillation period between $11.1 \mathrm{~s}$ and $14.5 \mathrm{~s}$, followed by cells whose oscillation period lies between $14.5 \mathrm{~s}$ and $18.8 \mathrm{~s}(\mathrm{n}=10)$. Also some marginal cases have an oscillation period higher than $18.8 \mathrm{~s}$. In our previous studies of periodic forcing [114] we found that the response with highest amplitude was given at a period of $T=20 \mathrm{~s}$. The discrepancy between the resonant frequency and the current period distribution suggests two possibilities: a) The response is nonlinear and amplitude dependent, and so the the amount of cAMP concentration will define the resonant frequency 


\subsection{PERIOD AND PHASE DISTRIBUTION}
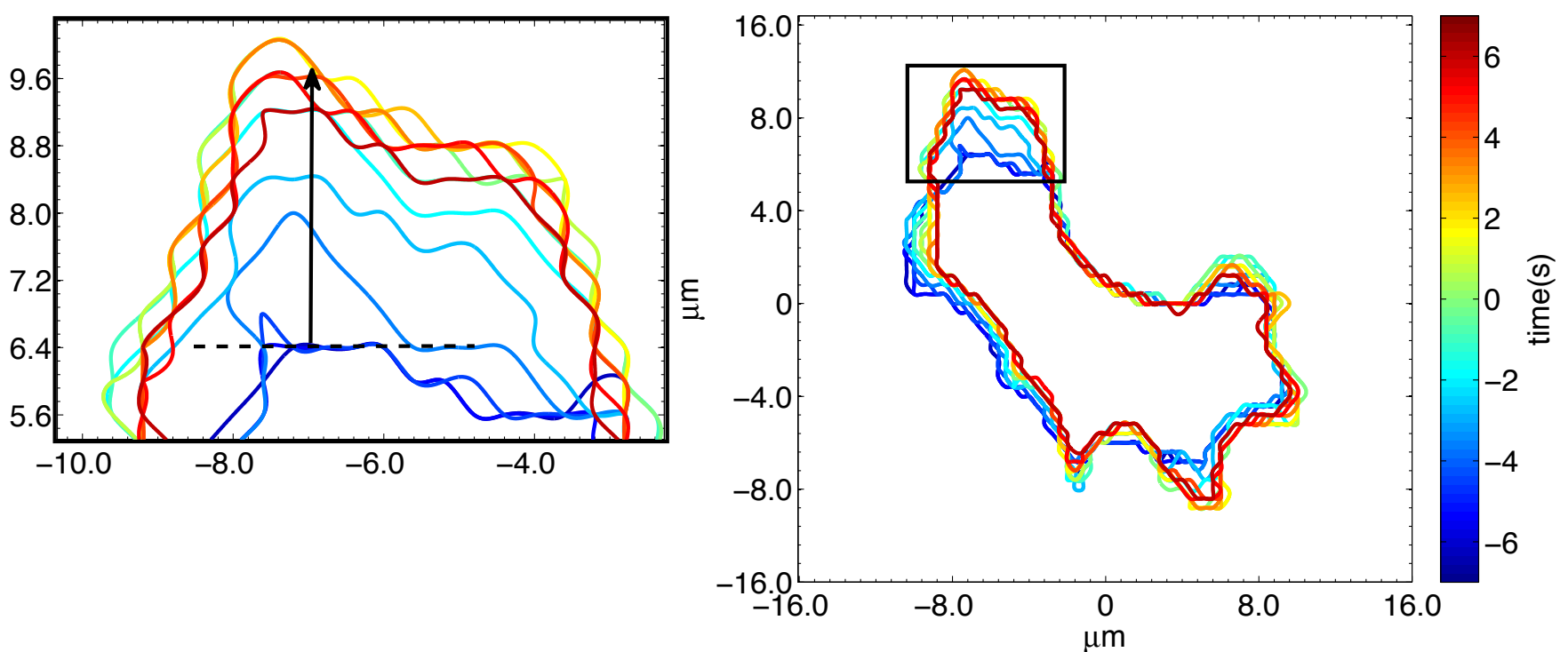

Figure 4.3: Contours extracting from a cell exhibiting self sustained oscillations (right panel), they correspond to a single period of oscillation. The color code corresponds to time where $t=0$ corresponds to the observed minimum value of LimE. The left panel corresponds to the pseudopod extended denoted by a black square at the right panel. 


\subsection{PERIOD AND PHASE DISTRIBUTION}
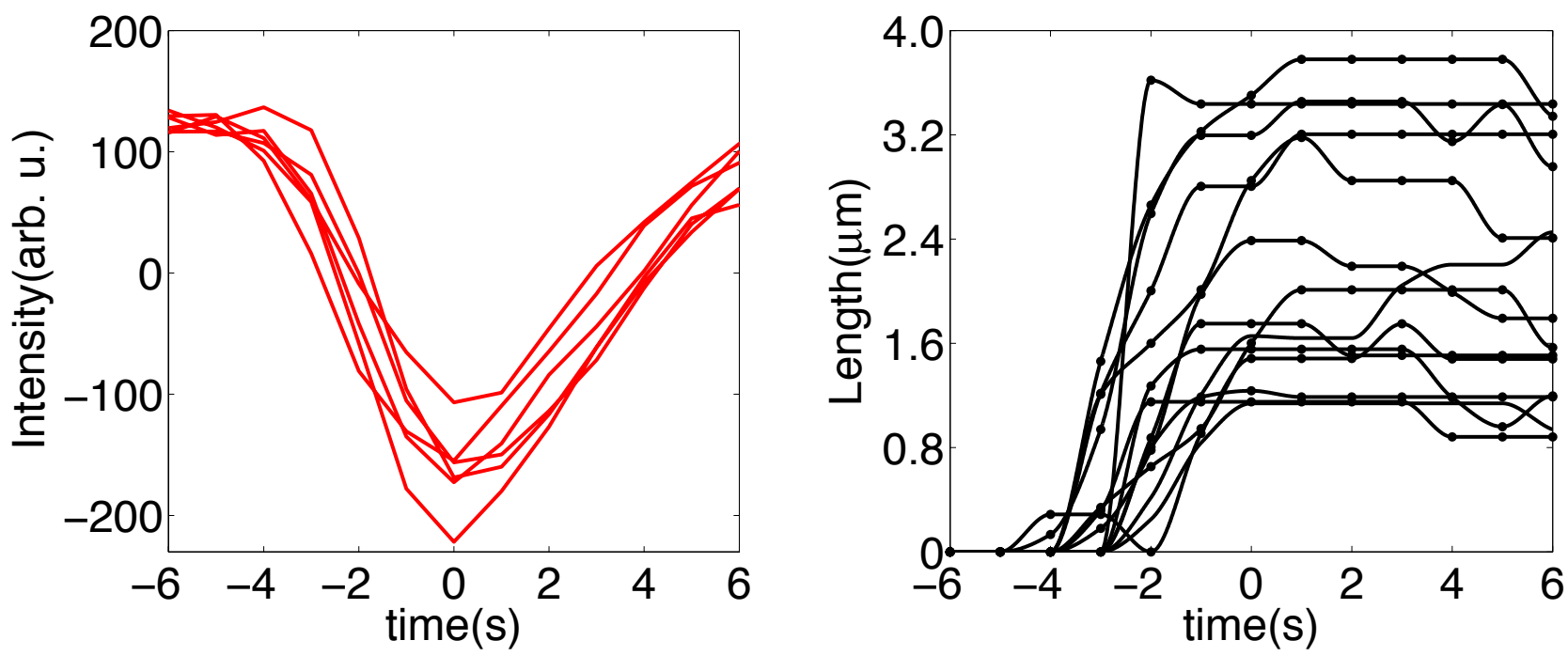

Figure 4.4: Relationship cytosolic LimE depletion and pseudopod length. The left panel corresponds to an overlap of LimE fluorescence depletion from a periodic time series, and the right panel are the time evolution of all the pseudopods observed in that measurement.
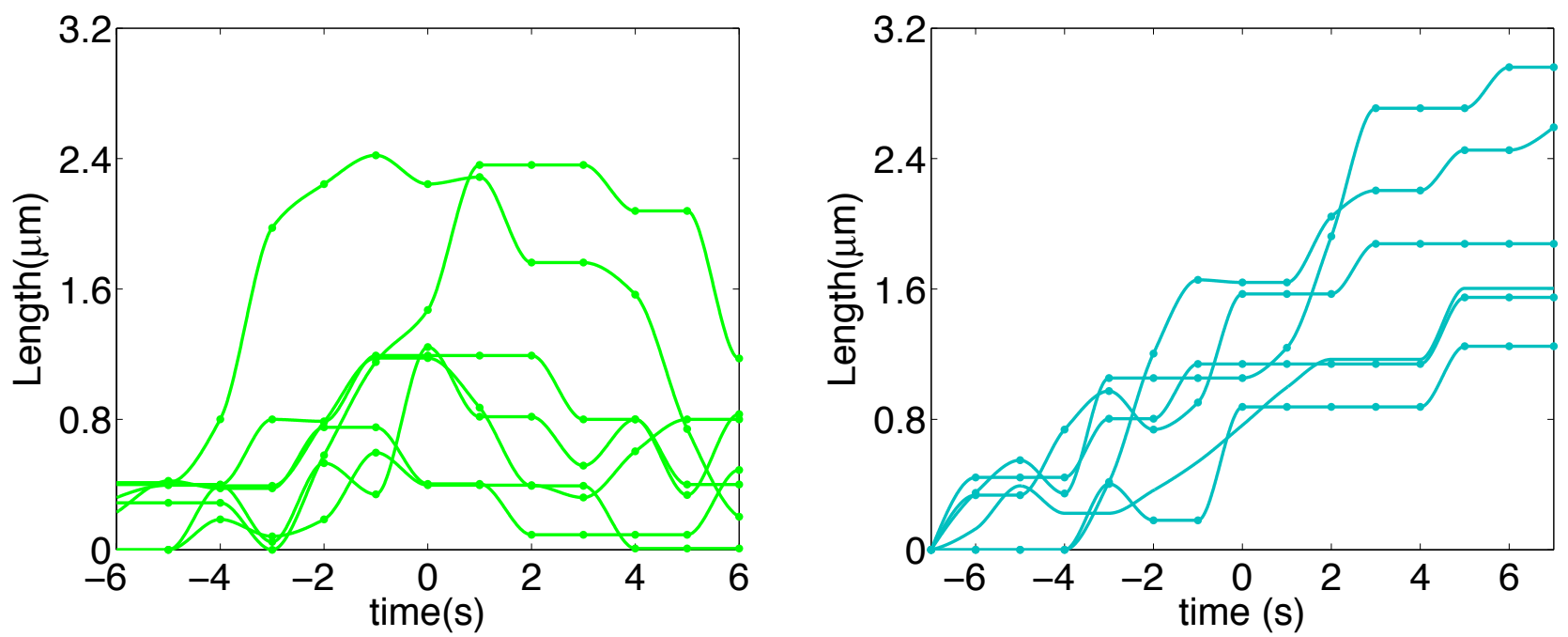

Figure 4.5: Time evolution pseudopod length extension-retraction (left panel) and the length of membrane retraction (right). 


\subsection{CORRELATION TIME $\tau_{C}$ AND VARIANCE $\sigma_{E X P}^{2}$}

(this line of research is currently investigated by $\mathrm{H}$. Hsu), or b) there is a time scale distribution for the components of the cytoskeleton and the signaling system, which would be reflected with different resonant and oscillation frequencies.

A dynamical system which exhibit periodic behavior needs at least two components [98], one acting as an activator and the other as an inhibitor, and they will exhibit a time delay with respect to each other. Also there is an alternative representation for a one component system, which is inhibited by a time delayed version of the same signal [74]. In both cases the inhibition profile is delayed, and therefore it is expected that the proteins that inhibit actin polymerization (such as Coronin 1A and Aip1) will be time delayed with respect to LimE. For example in Figure 4.2. Coronin $1 \mathrm{~A}$ has a time periodic profile which is delayed with respect to LimE. Therefore we have obtained the phase lag between Coronin 1A and LimE (Figure 4.7, left) and between Aip1 and LimE (Figure 4.7, right). In both histograms, except for two cells, their values span the negative side of the axis meaning that LimE is the leading signal for both proteins. The histogram for Coronin $1 \mathrm{~A}$ is peaked at $\Delta \phi=-1.1 \pi$ and its values span continuosly from $\Delta \phi=-1.58 \pi$ to $\Delta \phi=-.524 \pi$, with two marginal cases centered at $\Delta \phi=-.0511 \pi$. In the case of Aip1 the distribution is more uniform from $\Delta \phi=-1.84 \pi$ to $\Delta \phi=-0.28 \pi$ with also two separate cases centered at $\Delta \phi=-2.28 \pi$. The uniform distribution could be caused by the fact that is harder to measure an Aip1 signal since the label expression for Aip1-GFP tends to be weak and therefore difficult to measure. The phase lags supports the inhibitory role of Aip1 and Coronin 1A discussed in Chapter 2, though this topic will be studied more thoroughly in a Chapter 5 .

\subsection{Correlation time $\tau_{c}$ and variance $\sigma_{E x p}^{2}$}

As mentioned in Chapter 3, the autocorrelation function characterizes the statistical properties of a signal via the correlation time, which is a measure of signal coherence, and the variance, which is an indirect measure of amplitude. Figure 4.8 a shows the collected values for 31 cells ( $\mathrm{N}=12$ Aip1-GFP+LimE-mRFP and $\mathrm{N}=19$ Crn-GFP+LimE-mRFP) which showed at least one oscillation period in the autocorrelation function. There is a cluster of points limited by the values $\tau_{c} \sim 90 \mathrm{~s}$ and $\sigma_{\text {Exp }}^{2} \sim 0.25$ (denoted by a black triangle), this cluster of cells were 
4.4. CORRELATION TIME $\tau_{C}$ AND VARIANCE $\sigma_{E X P}^{2}$

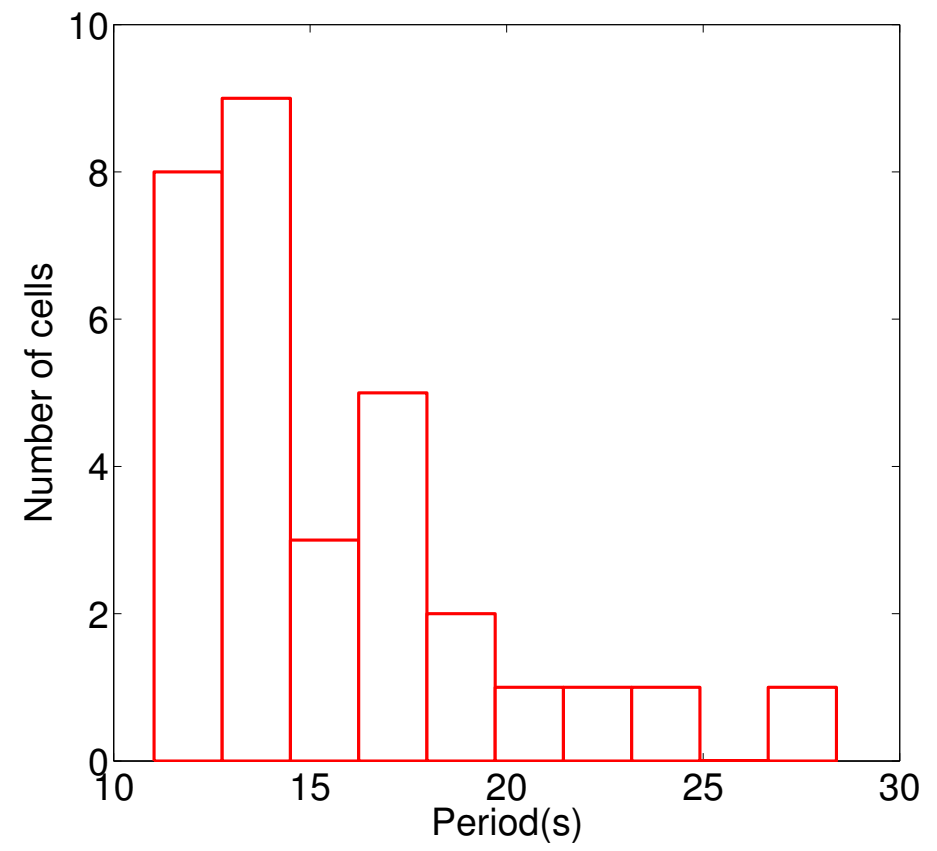

Figure 4.6: Period distribution for the observed LimE signals, $T=\frac{2 \pi}{\omega_{o}}$, taken from the measured autocorrelation functions. 
4.4. CORRELATION TIME $\tau_{C}$ AND VARIANCE $\sigma_{E X P}^{2}$
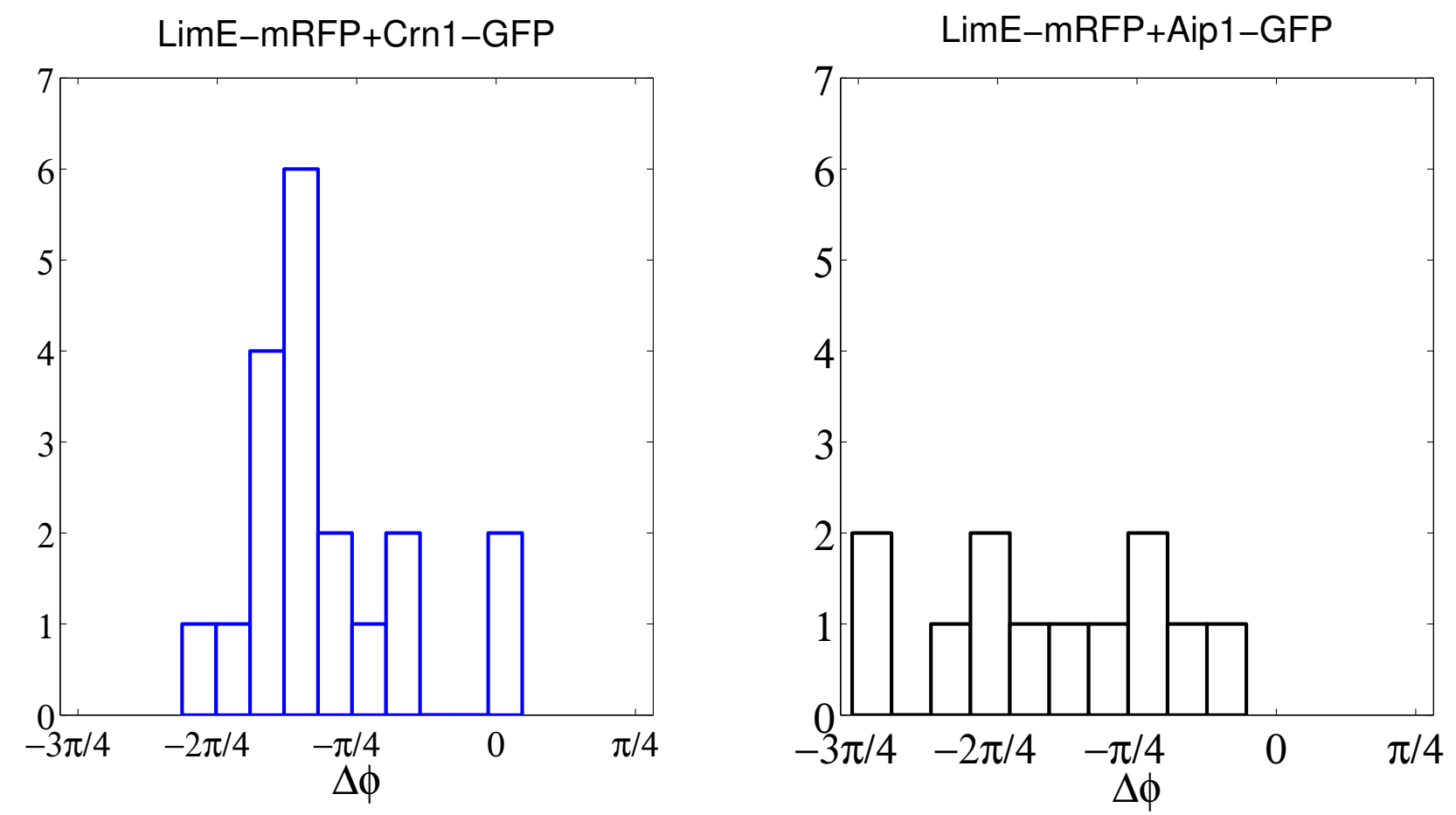

Figure 4.7: Phase lag distribution between LimE and Coronin 1A(left panel) and between LimE and Aip1 (right panel), taken from the measured cross correlation functions. 


\subsection{CORRELATION TIME $\tau_{C}$ AND VARIANCE $\sigma_{E X P}^{2}$}

classified as cells that exhibit short transients (defined and discussed in section 4.6). The outlying points from this region corresponds to cells that were classified as long transient cells (discussed also in section 4.6). The majority of the outlying cells have a correlation time one order of magnitude higher than in the cluster and therefore they are highly coherent. There is an apparent inverse relation between the correlation time and variance of these points, in which points with higher coherence exhibit lower amplitudes. Zooming into the area bounded by $\tau_{c} \sim 90$ s and $\sigma_{E x p}^{2} \sim 0.25$ also shows another cluster of points with lower variance and correlation time, but another set of points in this region follow an inverse relation.

After a careful analysis of these plots, we concluded that if we interpreted as if the observed oscillatory cells are all above the critical point, then they do not match the typical scenario that is expected. As the amplitude of an oscillator increases, the dissipation at the radial direction of phase space becomes stronger and amplitude fluctuations become negligible. All statistical properties are then given by the oscillators phase, these depends on the inverse of amplitude and therefore the correlation time increases as the amplitude increases. This would give as an expectation a monotonic increasing relation between correlation time and variance [60], which is followed in our plots only for some points. Therefore it is natural to ask, if actin polymerization is oscillating at above criticality, what are the conditions needed to get an inverse relations between correlation time $\left(\tau_{c}\right)$ and variance $\left(\sigma_{\text {Exp }}\right)$ ?

On the other hand, the distribution of correlation times might reflect the fact that some cells operate below the critical point. A nonlinear oscillator set to the parameter regime where it does not exhibit self sustained oscillations, would be fluctuating due to the forcing exerted by the environmental noise [11]. It would show a high cross correlation between its components, the autocorrelation function would show a finite frequency with a short correlation time [97]. As one of the control parameters is varied towards criticality, the oscillator becomes more sensitive to external fluctuations [116, 117, 16], close to the transition point the amplitude increases steeply to parameter variation [91. If this second scenario is true, then we can ask, how many cells are below/above critical point? and how much noise is contributing to the dynamics? In the next section, we discuss the minimum mechanisms necessary to reproduce our experimental observations. 


\subsection{CORRELATION TIME $\tau_{C}$ AND VARIANCE $\sigma_{E X P}^{2}$}
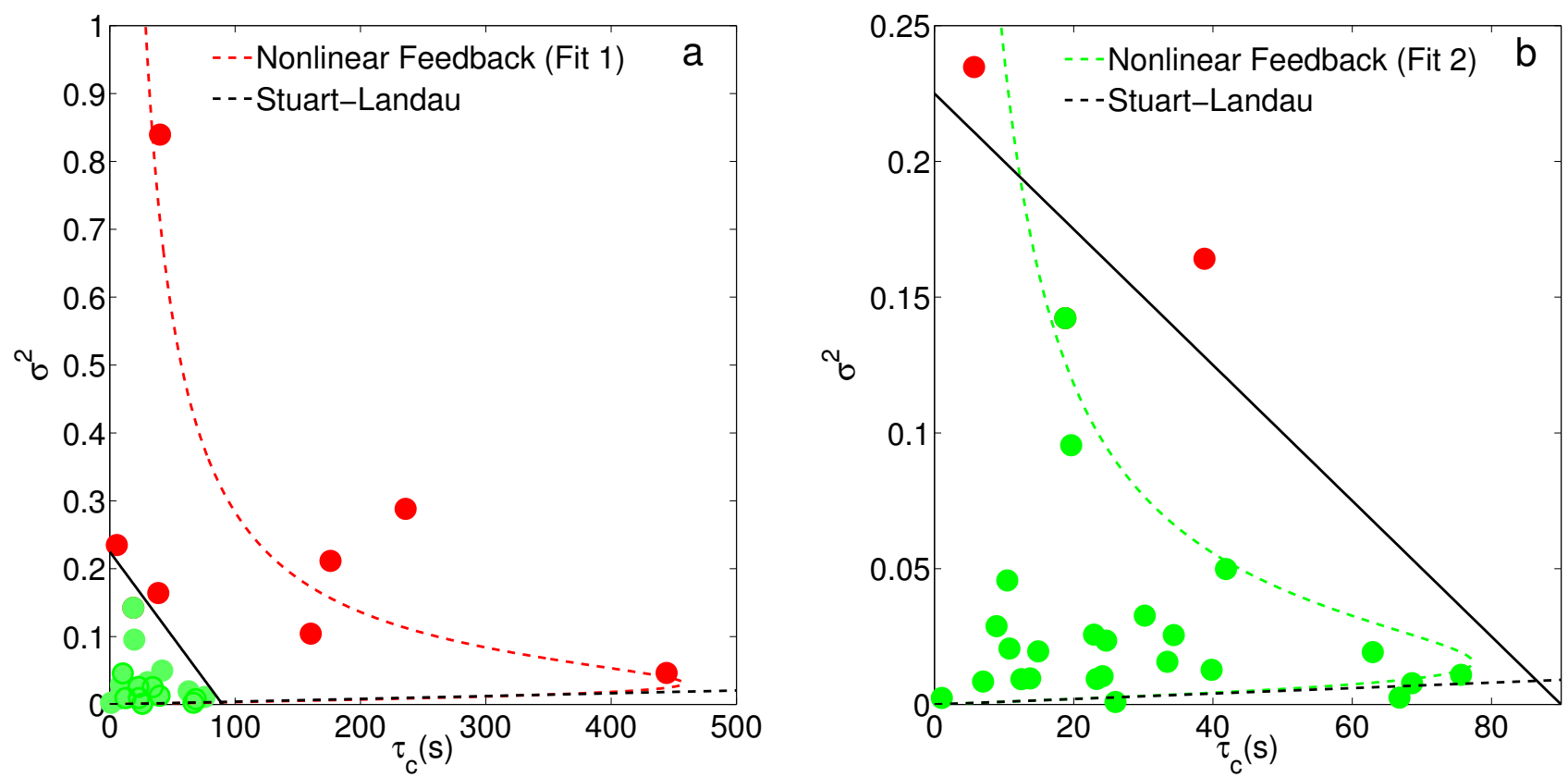

Figure 4.8: Relationship between correlation time $\left(\tau_{c}\right)$ and variance $\left(\sigma_{E x p}^{2}\right)$ extracted from the time series of cells with long transients (red) and with short transients (light green). a) Corresponds to all the parameters extracted from the time series, while b) is the amplification near the region bounded by a triangle in a. There are two curves fitted from Equation (4.11) using the parameters $D_{1}=0.0348$ $\& D_{2}=0.68 \times 10^{-4}$ (Fit 1) and $D_{1}=0.416 \& D_{2}=2.00 \times 10^{-4}$ (Fit 2) 


\subsection{ORIGIN OF THE STATISTICAL PROPERTIES OF ACTIN OSCILLATIONS.}

\subsection{Origin of the statistical properties of actin oscillations.}

As mentioned in the previous section, there is an apparent inverse relation between the correlation time of the LimE time series with its own variance. Though we can see in Figure 4.8 that the points are scattered through the $\left\{\tau_{c}, \sigma_{E x p}\right\}$ plane. To test how meaningful this scattering is, we proceed by doing a theoretical analysis of these signals. In this study we have tested systematically two hypotheses: In the first one, the internal parameters of the system are close to the transition point of an oscillatory instability, while at the same time the environment is affecting the actin cytoskeleton in the form of noise. The observed dynamics can be a consequence solely from the environments influence, but also from the internal mechanisms of actin polymerization. In the second the system is far from the transition point, where the amplitude is relatively insensitive to external noise and the statistical properties are given by the oscillators phase drift. In this case we also add a multiplicative noise term which as a consequence gives an inverse relation between correlation time and variance.

\subsubsection{Close to an oscillatory instability with additive noise}

We start by analyzing a canonical model for self oscillating processes called the Stuart-Landau model [83] subject to external fluctuations

$$
\frac{d z}{d t}=\left(\lambda+i \omega_{o}-\epsilon|z|^{2}\right) z+\sqrt{2 D} \xi(t)
$$

This is a generic model for the emergence of self sustained oscillations and is defined in the complex plane where $z(t)=x(t)+i y(t)$. The parameters $\lambda$ and $\epsilon$ are the linear and nonlinear dissipation rates respectively, $\omega_{o}$ is the eigenfrequency of the oscillator, $\xi(t)$ is an stochastic variable defined as $\left\langle\xi_{i}(t-\tau) \xi_{i}(t)>=\delta(t)\right.$, and $D$ characterizes the noise variance. The parameter $\lambda$ controls the dynamical state of the system, when $\lambda<0$ the system is stable and when perturbed from its equilibrium it returns to its basal state. As the parameter is increased to $\lambda>0$ the basal state becomes unstable and the amplitude of the system increases to a finite value where the input energy is balanced by the nonlinear dissipation. 


\subsection{ORIGIN OF THE STATISTICAL PROPERTIES OF ACTIN OSCILLATIONS.}

When $D>0$ it corresponds to the Langevin version and presents fluctuations in both regimes. These fluctuations are strongly amplified near the transition point [116, 117] and these properties have been used to model the amplification properties of the cochlea [16].

The dynamics of the system close to the transition point, can be studied by solving the Fokker-Planck equation corresponding to the amplitude equation [91] obtained by using $z(t)=a(t) e^{i \phi(t)}$ (for the remainder of the analysis we set $\epsilon=1$ ). This has an exact solution for the probability distribution function (PDF) of the squared amplitude

$$
P\left(a^{2}\right)=\frac{1}{\sqrt{\pi D}\left(1+\operatorname{erf}\left(\frac{\lambda}{2 \sqrt{D}}\right)\right)} \exp \left[\frac{-1}{4 D}\left(a^{2}-\lambda\right)^{2}\right]
$$

Notice that this expression already provides a way to classify graphically in which dynamical state the cells are. Since the amplitude is defined only for positive values, Equation 4.5 will have a well defined maxima when $\lambda>0$, while for $\lambda<0$ it will correspond to a monotonically decaying function. Figure 4.9 shows an example of two cases in which the squared amplitude was extracted from two experimental LimE signals using the Hilbert Transform. The distribution of Figure 4.9 A has a well defined maxima, we can infer that actin in this case would exhibit self oscillations in the absence of noise, and the spreading of the distribution reflects the external noise intensity. The second distribution (Figure 4.9 B) is a monotonically decaying function and therefore in this case actin polymerization is only driven by noise.

The distribution function in Equation 4.5 is effectively a Gaussian with the mean set to $\lambda$ and a variance of $2 D$. We extracted the parameters of this PDF using the NLSF algorithm, as it was used for the autocorrelation function in section 4.4. The parameters could not be extracted in all cases as the algorithm would find a large confidence interval when the distributions were monotonic. In this case we fitted the limiting case $P\left(a^{2}\right) \approx C_{o} \exp \left[\frac{\lambda}{2 D} a^{2}\right]$, in which the decay rate gives the ratio between $\lambda$ and noise variance. To compare this hypothesis with the experimental data, we make use of the predicted signal variance calculated by using Equation 4.5 


\subsection{ORIGIN OF THE STATISTICAL PROPERTIES OF ACTIN} OSCILLATIONS.

$$
\sigma_{T}^{2}=\lambda+\sqrt{\frac{4 D}{\pi}} \frac{1}{\left(1+\operatorname{erf}\left(\frac{\lambda}{2 \sqrt{D}}\right)\right)} \exp \left[-\left(\frac{\lambda}{2 \sqrt{D}}\right)^{2}\right]
$$

where $\sigma_{T}^{2}$ stands for theoretical or predicted variance. By using the parameters $\lambda$ and $\sqrt{D}$, in which NLSF algorithm was able to extract separately, we constructed the value $\sigma_{T}^{2}$.

Figure 4.10 A shows the ratio between the variance obtained directly from the experimental signals $\sigma_{\text {Exp }}^{2}$ and the predicted variance $\sigma_{T}^{2}$ against the dimensionless parameter $\frac{\lambda}{\sqrt{D}}$. The majority of the cells lie very close to the $\frac{\sigma_{E x p}^{2}}{\sigma_{T}^{2}}=1$ line with an agreement of $\sim 20 \%$ for all but 7 cells. The blue crosses in Figure $4.10 \mathrm{~A}$ corresponds to LimE-GFP data provided by H. Hsu. To see how much noise influenced the dynamics of self polymerizing cells, we defined the Relative Noise Contribution $(R N C)$, which is the percentage of amplitude contributed by the noise

$$
R N C=\frac{\sigma_{E x p}^{2}-\sigma_{D e t}^{2}}{\sigma_{E x p}^{2}}
$$

where $\sigma_{D e t}^{2}=\lambda \Theta(\lambda)$ is the deterministic contribution to the variance and $\Theta($. corresponds to the Heaviside function. For negative values of $\lambda$ the $R N C=1$, since only noise contributes to the observed dynamics. There is a remarkably good agreement between the measured $R N C$ and its theoretical prediction (Figure 4.10 B). Notice that the majority of the cells shown are below the value $\frac{\lambda}{\sqrt{D}}<1$, where noise contributes at least to $35 \%$ of the amplitude. Solving $\sigma_{T}=\sigma_{E x p}$ we have determined the values of $\lambda$ and $\sqrt{D}$ for the rest of the cases, Figure 4.10 $\mathrm{C}$ restates that the majority of the cells lie in the $\frac{\lambda}{\sqrt{D}}<1$ region, and according to this hypothesis the majority of the actin oscillations are predominantly driven by noise.

\subsubsection{Multiplicative noise and far from the oscillatory in- stability point}

As mentioned before in the materials and methods chapter, when the amplitude is high enough, the correlation time of an oscillator is defined by the phase drift [60]. 


\subsection{ORIGIN OF THE STATISTICAL PROPERTIES OF ACTIN OSCILLATIONS.}
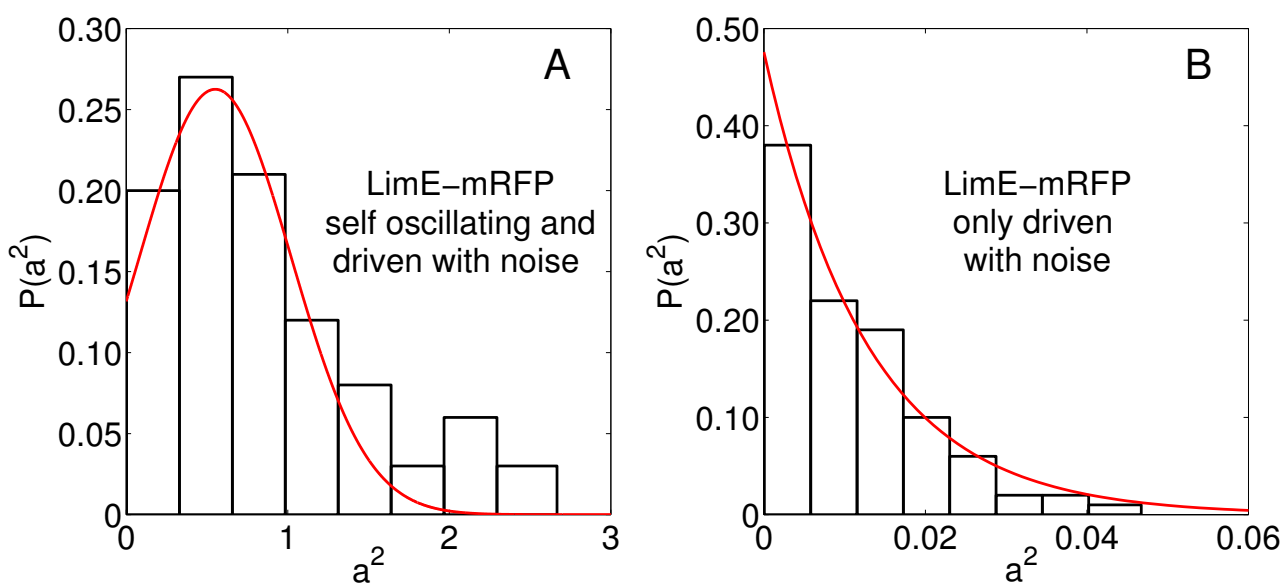

Figure 4.9: Examples of squared amplitude distributions for two different LimEmRFP signals. A) The distribution function has a well defined maxima and a Gaussian distribution can be fitted, according to Equation 4.5 corresponds to an cell self-oscillating driven by noise at the same time. B) Monotonically decaying distribution function corresponding to case where actin is only driven by noise.

By rewriting Equation (4.4) in polar coordinates we arrive to the phase equation

$$
\frac{d \phi}{d t}=\omega_{o}-\sqrt{\frac{2 D}{\sigma^{2}}} \xi(t) \sin (\phi)
$$

where $\sigma^{2}$ is the variance of $z, a_{o}$ is the steady state amplitude, and the approximation $\sigma^{2} \approx a_{o}^{2}$ is used. Notice from Equation 4.8 that when the variance increases the effects of external noise becomes weaker. As mentioned in Chapter 3 the correlation time $\tau_{c}$ quantifies the influence of phase fluctuations, so at high variance values the phase drift decreases resulting in higher correlation times. In that case there will be a monotonically increasing relation between variance and correlation time. This system cannot reproduce our experimental observations since we have observed inverse relations between these two parameters (Figure 4.8).

To overcome this drawback we introduced two noise sources in Equation (4.4), one multiplicative and one additive. The resulting equation is of the form

$$
\frac{d z}{d t}=\left(\lambda+i \omega_{o}-\epsilon|z|^{2}-i \sqrt{2 D_{1}} \xi_{1}(t)|z|\right) z+\sqrt{2 D_{2}} \xi_{2}(t)
$$




\subsection{ORIGIN OF THE STATISTICAL PROPERTIES OF ACTIN OSCILLATIONS.}
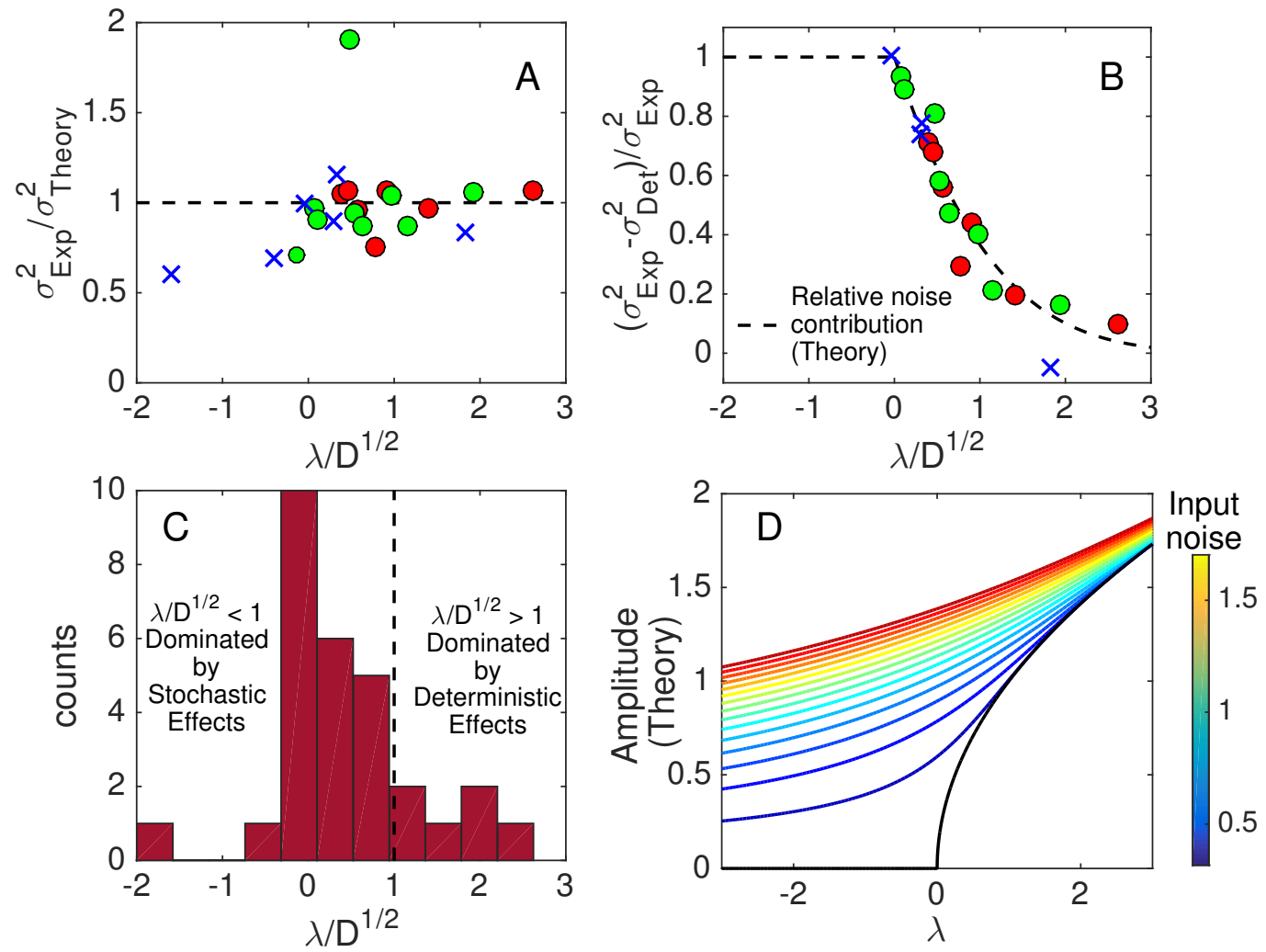

Figure 4.10: A) Comparison between the calculated $\left(\sigma_{T}^{2}\right)$ and the measured $\left(\sigma_{E x p}^{2}\right)$ of signal variance. B) Relative noise contribution $(R N C)$, the dashed black line corresponds to the the theoretical prediction. C) Histogram for $\lambda / \sqrt{D}$ showing that for the majority of the cells actin is driven by noise. D) Theoretical calculation of amplitude around the critical point $\lambda=0$ with various noise intensities, the black line corresponds to $D=0$. 


\subsection{ORIGIN OF THE STATISTICAL PROPERTIES OF ACTIN OSCILLATIONS.}

where $D_{2}$ is the variance of the additive noise and $D_{1}$ is the variance of the introduced multiplicative noise. Notice that the multiplicative term is coupled with $|z|$ making it amplitude dependent and is also multiplied by the imaginary number $i$, indicating that it influences the feedback each component senses. The resulting phase equation for this case is

$$
\frac{d \phi}{d t}=\omega_{o}-\sqrt{2 D_{1} \sigma^{2}} \xi_{1}(t)-\sqrt{\frac{2 D_{2}}{\sigma^{2}}} \xi_{2}(t) \sin (\phi)
$$

We can observe that the multiplicative term in Equation 4.9 will induce higher phase drifts as the variance increases.

Our measurements of LimE are related to the variable $x(t)$, while $y(t)$ is the effective inhibiting feedback that LimE senses correlated to either Coronin $1 \mathrm{~A}$ or Aip1. As we have reviewed in Chapter 2, Coronin 1A and Aip1 behaves as an effective inhibitor for the cytoskeleton as they enhance the disassembly of actin filaments. Also in section 4.3 we have shown that Aip1 and Coronin 1A show the phase lag expected for inhibitors. In Figure 4.11 (left) we have an schematic of the model Equation 4.9 with its two noise sources. The role of the multiplicative term is to act as an internal noise source that corrupts the feedback between the two nodes. The second noise source is an external one, which represents either the ambient thermal noise or an stochastic signal coming from another module in the cells biochemical network. In Figure 4.11 (right) there is the comparison of a numerical realization of Equation (4.10) (x-red, y-blue), and a measured time series (LimE-red, Coronin 1A-blue). It can be seen that several features of the experimental data is reproduced by the model.

To validate this model, an analytical expressions for the relation between correlation time and variance were obtained. From Equation (4.10) we can derive an expression valid at high amplitudes

$$
\tau_{c}=\frac{\sigma^{2}}{2 D_{1} \sigma^{4}+D_{2}}
$$

details on the derivation are given in Appendix A. In Figure 4.8 we fitted Equation (4.11) for the outlying points where the majority show highly coherent oscillations with the parameters $D_{1}=0.0348 \& D_{2}=0.68 \times 10^{-4}$, while for cells with low 


\subsection{ORIGIN OF THE STATISTICAL PROPERTIES OF ACTIN OSCILLATIONS.}

correlation times we used $D_{1}=0.416 \& D_{2}=2.00 \times 10^{-4}$ (the parameters were fitted by hand). The black dashed line corresponds to the case were there is only an additive noise source (Stuart-Landau equation), we can see that for low variance values the relation between correlation time and variance is linear. As the variance increases the deviation from the linear relation becomes more apparent, the effects of the multiplicative noise turn important for giving an inverse relation. Therefore we concluded that having multiplicative (internal) noise in addition with the additive (external) noise is a plausible scenario to explain the observed properties actin polymerization.

\subsubsection{Interpretation of both scenarios}

Both hypotheses presented in this section are plausible scenarios on how noise influences actin polymerization. Our measured time series are short, and it is possible that this is inducing artifacts in our parameter estimation. Therefore we resorted to do a numerical study to reproduce short time series with Equation 4.4 to realize how well the variance $\sigma_{\text {Exp }}^{2}$ and correlation time $\tau_{c}$ are estimated (see Appendix B for details). The correlation time $\tau_{c}$ is not well estimated in short time series, as the dispersion of values obtained from several single simulations is large (details in Appendix A). It still provides a measure of coherence and the ensemble average converges to the theoretical prediction, but the number of measurements needed to compare it with any model would be too high. On the other hand the dispersion in variance $\sigma_{E x p}^{2}$ is not as high and is estimated well for short time series. With this in mind and the good agreement in Figures $4.10 \mathrm{~A}$ and $4.10 \mathrm{~B}$, we concluded that the actin cytoskeleton of $D$. discoideum operates very close to the onset of a dynamical instability (as conjectured before [114]). The strength of noise is high for the majority of cells exhibiting self sustained oscillations, where it contributes to at least $35 \%$ of the amplitude. It might be possible that $D$. discoideum takes advantage of this sensitivity in order to keep high amplitudes at low metabolic spending, where this is represented by low or negative values of $\lambda$ (see Figure $4.10 \mathrm{D}$ ). 
4.5. ORIGIN OF THE STATISTICAL PROPERTIES OF ACTIN OSCILLATIONS.
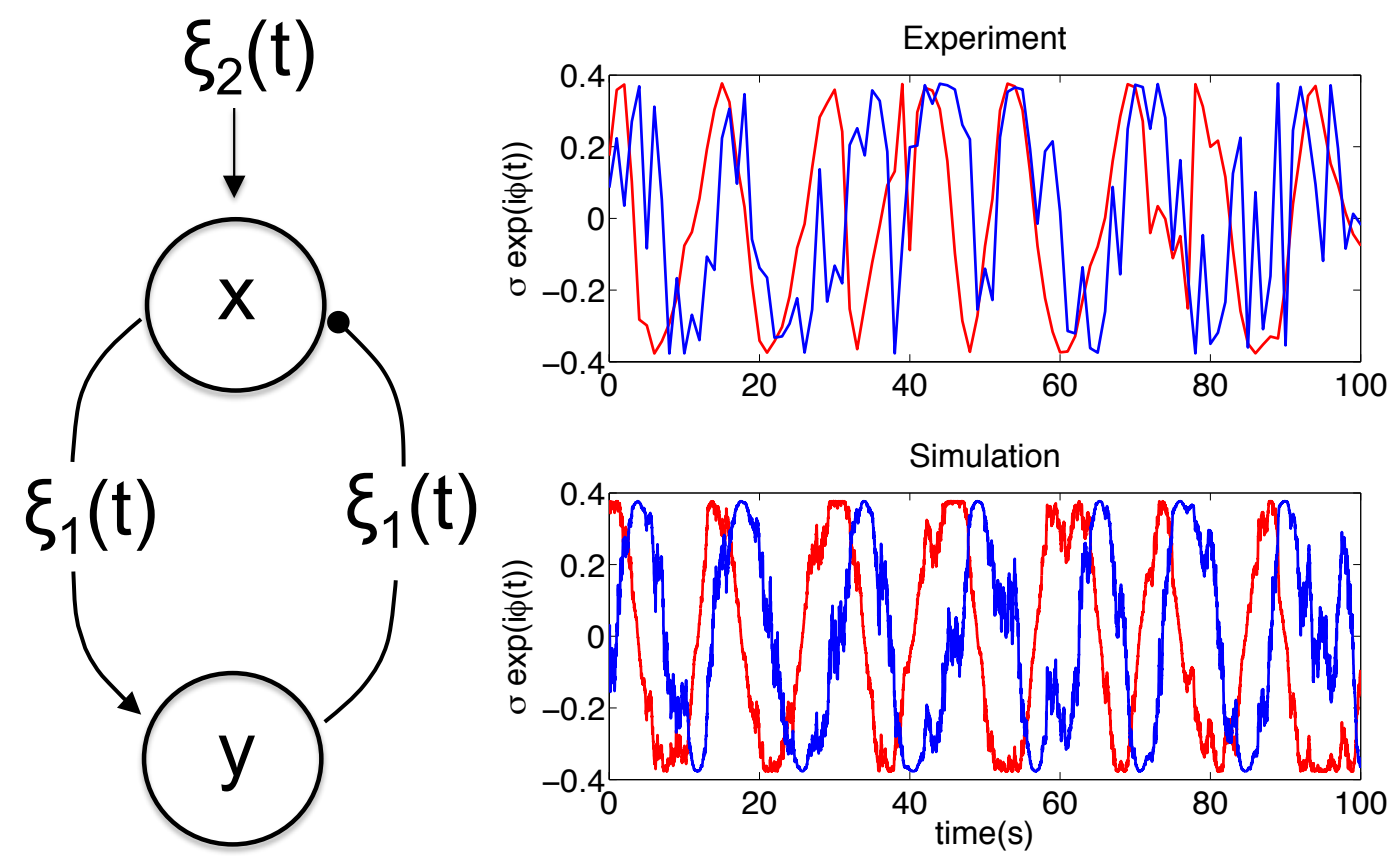

Figure 4.11: Left, schematic of the two variable model Equation (4.9). Right, comparison between a measurement and simulation from Equation (4.10). The simulation was performed with the Euler-Maruyama method [47] (Time step $\Delta t=$ 0.01, $\sigma^{2}=0.1423, D_{1}=0.416$ and $\left.D_{2}=2.00 \times 10^{-4}\right)$. 


\subsection{TRANSIENT RESPONSES}

\subsection{Transient responses}

During our analysis we found that cells classified as oscillatory respond differently to cAMP stimulation. Figure 4.12 shows the time series for two different oscillatory cells labeled with LimE-mRFP+Crn-GFP, at $t=100 \mathrm{~s}$ both cells were stimulated (marked by a black arrow in both plots). At first inspection of Figure 4.12 a, we can see that the time series are noisy, oscillatory and cross correlated. The coefficients characterizing the time series are $\tau_{c}=13.75 \mathrm{~s}$, with a main period of $T=14.66 \mathrm{~s}$ and the maximum cross correlation value is 0.61 . When the cell is stimulated we can see that there is a transient response, the values of LimE decreases and then overshoots, and in the same manner Coronin 1A does the same. This is the characterizing LimE response observed in our previous study [114]. After $30 \mathrm{~s}$ of stimulation the previous fluctuating activity continues with no apparent signature of the previous stimulation (other examples are shown in Appendix C, Figure C.2.). In Figure 4.8 and 4.10 these cells correspond are marked in light green.

Some cells presented stationary nearly periodic activity in their cytosolic fluorescence and responded differently. Figure $4.12 \mathrm{~b}$, shows the time series of a cell with such activity, with a correlation time of $\tau_{c}=236 \mathrm{~s}$ which is one order of magnitude higher than in the previous case. The maximum cross correlation value is 0.91 and it oscillates with a period of $T=14.28 \mathrm{~s}$. After stimulation this cell shows a transient response which terminates completely the observed oscillations. At around $100 s$ after stimulus, the oscillation reappears with the amplitude gradually increases, and it takes around four oscillation periods to recover. In Figure C.1 of Appendix C we have time series of six out of the seven cells that exhibited long transients, these cells are represented in Figure 4.8 and 4.10 with a red marks. We noted that all the cells with this type of response have a positive values of $\frac{\lambda}{\sqrt{D}}$,

even though positive values of $\frac{\lambda}{\sqrt{D}}$ is not a precondition for this (see Figure 4.10. The gradual amplitude recovery around a well defined mean is a trademark of a Hopf Bifurcation [44, though the halting and subsequent recovery of these oscillations can be due to several mechanisms. In the next section we systematically analyze the possible scenarios giving rise to these responses, as they reveal some of the principles behind them. 


\subsection{MODELING LONG TRANSIENTS}
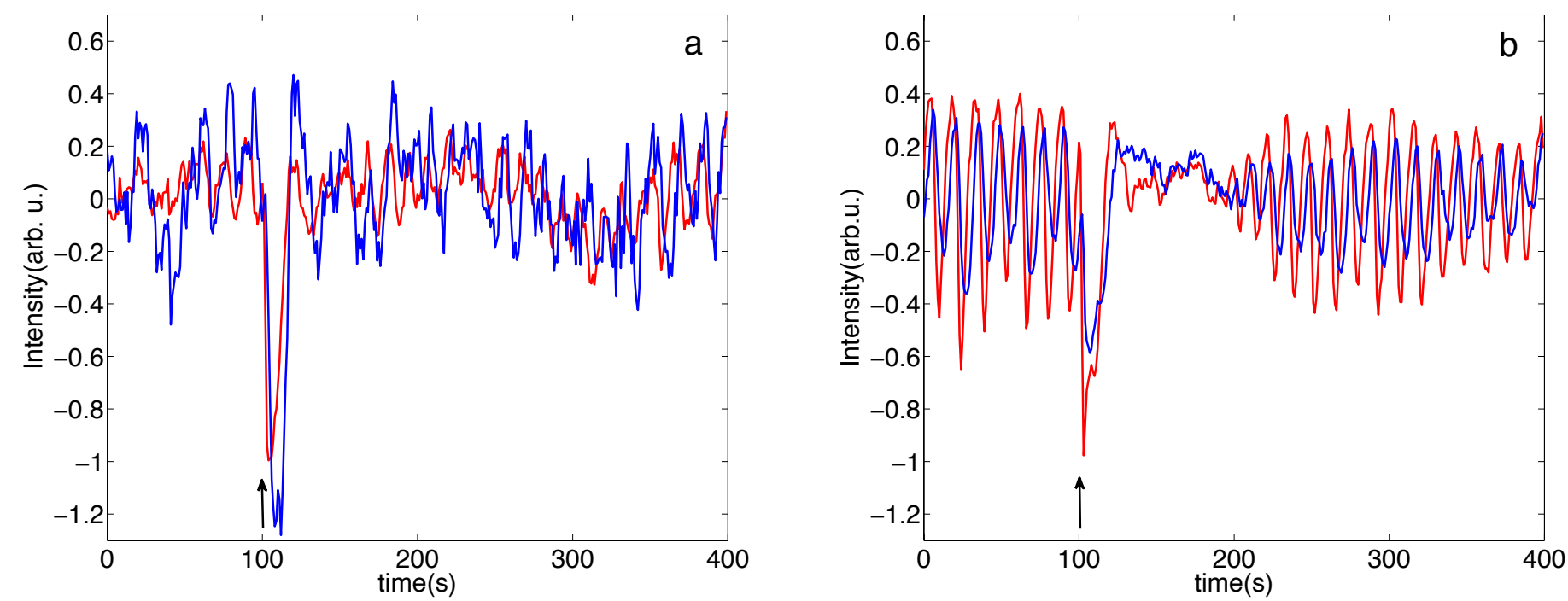

Figure 4.12: Different observed transients, a) corresponds to a cell with short transient and low correlation time and b) to a cell with a long transient and high correlation time (Red,LimE-mRFP and Blue, Cor-GFP ).

\subsection{Modeling long transients}

\subsubsection{Phase portraits of different scenarios}

This chapter finalizes with the analysis of the dynamics implicit in the appearance of long transients, such as those shown in Figure $4.12 \mathrm{~b}$. First we notice the form of the amplitude response extracted from a single cell. Figure 4.13 shows the time evolution for the amplitude of an oscillating LimE-mRFP+Coronin 1A-GFP cell with a long transient response. We defined amplitude as

$$
a(t)=\sqrt{\operatorname{LimE}(t)^{2}+\operatorname{Coronin} 1 A(t)^{2}}
$$

At $\mathrm{t}=100 \mathrm{~s}$ the stimulation is applied, before it the amplitude is fluctuating around a constant value. Then as the cell is stimulated, the amplitude increases sharply to then decrease to a lower value than the original base line. This time evolution is representative for all cells since it was always observed that after stimulation 


\subsection{MODELING LONG TRANSIENTS}

the amplitude always increases.

To understand what this pulse implies we will use geometrical arguments on the possible phase portraits to disentangle the dynamics behind it. We start by defining a generalized model for a self sustained oscillator in normal form

$$
\frac{d z}{d t}=\left(f(|z|)+i \omega_{o}\right) z
$$

where $z=x+i y$ and $\omega_{o}$ is the natural frequency. By standard linearization methods we get 44]

$$
\frac{d z}{d t} \approx\left(\frac{\left|z_{o}\right|^{2}}{2} \frac{\partial f\left(\left|z_{o}\right|\right)}{\partial|z|}+f\left(\left|z_{o}\right|\right)+i \omega_{o}\right) z
$$

which corresponds to an eigenvalue equation. We can see that the real part of the eigenvalue is $\Re e[\lambda]=\frac{\left|z_{o}\right|^{2}}{2} \frac{\partial f\left(z_{o}\right)}{\partial z}+f\left(\left|z_{o}\right|\right)$ and determines the stability of the amplitude at each point $z_{o}$. If the eigenvalue is positive around $z_{o}$ the amplitude will be unstable and if negative it will be stable. Therefore the form of $f(|z|)$ will determine under which conditions a finite amplitude oscillation becomes unstable towards a quiescent state.

Without loss of generality we start by defining a first model by choosing

$$
f(|z|)=-\lambda+\epsilon|z|^{2}-\eta|z|^{4}
$$

in Figure 4.14 (left) we have the corresponding phase portrait for $\lambda=1, \epsilon=2$, $\eta=0.5$ and $\omega_{o}=0.8$. In the phase portrait we have two basins of attraction divided by an unstable limit cycle (denoted by a brown circle). The vector fields converge either to a stable limit cycle or to a stable fixed point, therefore the two regimes observed experimentally (Figure $4.12 \mathrm{~b}$ ) are accessible in this model. The interpretation is readily available by looking at the phase portrait, if the concentrations of LimE and its inhibitor are driven to a certain minimum value the reaction cannot be sustained anymore and the oscillation dies out.

This phase portrait already gives us a prediction on the characteristic amplitude time series we must observe if this case is true. The stable fixed point is at the center of both limit cycles, and in order to reach it, any trajectory must start inside the unstable cycle which at the same time is inside the stable one. This would 


\subsection{MODELING LONG TRANSIENTS}

be represented in the time series as a decrease in amplitude before reaching the new equilibrium level after being perturbed externally. Figure 4.15 (left) shows a numerical realization of this equation forced by both additive noise and an external short time perturbation (details on the numerical simulations performed in this work are given in Appendix B). It can be seen that the amplitude shows no pulsatile response when making a transition to the new equilibrium level. We argue that independently of the nonlinearity or model, a two component differential equation cannot reproduce the observed dynamics in Figure $4.12 \mathrm{~b}$. This is due to the fact an equilibrium level inside the limit cycle will never be reached by perturbing the variables outside of it, and the vector field in the phase portrait cannot cross with itself [44, 98]. Experimentally we have observed that addition of cAMP always increases the amplitude independently of the transient response. Using other nonlinearities in Equation 4.14 will deviate the shape of limit cycles from circle to another closed form, or establish more fixed points and limit cycles, but it would be impossible to create a topology that creates the experimentally observed scenario.

Therefore by using this new conclusion we extend the initial model by adding more variables

$$
\begin{array}{r}
\frac{d z}{d t}=\left(f(|z|, w)+i \omega_{o}\right) z \\
\frac{d w}{d t}=g(z, w)
\end{array}
$$

where again without loss of generality we define the following functions

$$
\begin{array}{r}
f(|z|, w)=-\lambda(|z|, w)-\epsilon\left|z^{2}\right| \\
g(z, w)=-\alpha w+\frac{\beta}{2}(1+\lambda(|z|, w)) \\
\lambda(|z|, w)=\tanh \left(\eta\left[w+\frac{w_{o}}{z_{o}}|z|-w_{o}\right]\right)
\end{array}
$$

By defining amplitude as $a(t)=|z(t)|$, Figure 4.14 (right) shows a phase portrait given the parameters $\epsilon=1, \alpha=1, \beta=0.75, \eta=100, w_{o}=1.5$ and $z_{o}=1.0$. This 


\subsection{MODELING LONG TRANSIENTS}

phase portrait is divided in two basins of attraction making the system bistable, with one attracting fixed point at $a^{*}=1.0$ and $w^{*}=0.0$ and another at $a^{*}=0.0$ and $w^{*}=1.5$. Also in this case, as in the previous, the experimentally observed stable states are also available. From the phase portrait it can bee seen that is possible to reach $a^{*}=0.0$ starting from $a^{*}=1.0$ by perturbing the amplitude $a(t)$ to higher values. Figure 4.15 (right) shows a numerical realization of Equation (4.16), after the perturbation there is a pulse before decreasing to the second stable value. Therefore three components are needed at least, to reproduce the transition to low amplitudes as observed in the experiments.

The amplitude might be recovered after stimulation by two possible simple mechanisms. In the first one, $w$ can be affected by external noise inducing transitions back to the initial basin of attraction where $a^{*}=1.0$ resides. If we allow noise to be the recovery mechanism, then in the same manner we should observe sudden amplitude decreases. Since the system is bistable such systems noise induced transitions should be observed be observed [45]. In all performed experiments so far we have not observed a cell whose actin is polymerizing (oscillating) prominently and then stopping its activity without external cAMP stimulation. Since we find this mechanism unlikely we proposed another possibility, a deterministic one. It can be obtained by adding a variable $v$, which feeds from $w$, and at the same time inhibits it slowly, the coupling would be of the form

$$
\begin{gathered}
\frac{d w}{d t}=g(z, w)-v \\
\frac{d v}{d t}=\frac{1}{\tau}(w-v)
\end{gathered}
$$

A numerical realization with $\tau=33.33 \mathrm{~s}$ of such a system is shown in Figure 4.16 a, we have in the top panel the dynamics of the observed activator and inhibitor and the inferred variables $w$ (black) and $v$ (green). From these dynamics we can see an interplay between fast and slow time scales as were mentioned at the introduction for the signaling cascade and the actin cytoskeleton. 


\subsection{MODELING LONG TRANSIENTS}

\subsubsection{Interpretations for the fast and slow time scales}

The model of Equations 4.16 and 4.18 shows that the state of the oscillator is controlled by external variables operating at slower time scales. Assuming modularity in the coupled signaling/actin cytoskeleton system, we can associate the slow time scales to the signaling system (e.g. PTEN/PIP3) and the fast time scales with the actin cytoskeleton [48]. This is supported by the slow waves (in the order of $t=100 \mathrm{~s}$ ) observed for the signaling system when the cells are treated by Latrunculin A [4, 102, 48]. In these experiments the actin cytoskeleton is completely decoupled from the signaling system, and it would be worth to observe the times scales of PTEN/PIP3 when coupled to it. For the moment we do not have such data for self oscillating cells, but still we can discuss possible predictions. If the modularity assumed of the signaling cascade/actin cytoskeleton is valid, then PTEN and PIP3 would present dynamics with similar time scales to those of $v$ and $w$. But since the signaling cascade and actin cytoskeleton are coupled it is possible that both fast and slow time scales would be observed in PTEN and PIP3.

In order to see how could this happen, we construct a model easily using Equations 4.16 and 4.18 as a basis for a new model. We change the coupling between the variables by changing the coordinate system of the observed phase space from $\vec{g}=\{x, y, v, w\} \rightarrow \overrightarrow{g^{\prime}}=\left\{x^{\prime}, y^{\prime}, v^{\prime}, w^{\prime}\right\}$. This is achieved easily by a linear transformation

$$
\overrightarrow{g^{\prime}}=\mathbf{M} \vec{g}
$$

As an example we can use the following matrix to induce an arbitrary transformation

$$
\mathbf{M}=\left(\begin{array}{cccc}
1 & 0 & 0 & 1 \\
0 & 1 & 1 & 0 \\
0 & -1 & 1 & 0 \\
-1 & 0 & 0 & 1
\end{array}\right)
$$

which we have used to transform the time series from Figure $4.16 \mathrm{a}$, into the time series of Figure 4.16 b. The exact form of the new ODE model $\left(\frac{d \overrightarrow{g^{\prime}}}{d t}=f\left(\overrightarrow{g^{\prime}}\right)\right)$ is of no interest to us, but only the fact that all the variables in the new model 


\subsection{CHAPTER SUMMARY}

are coupled to each other. Notice in Figure 4.16 b that all four variables exhibit both slow and fast time scales. This shows that if the components of the actin cytoskeleton and the signaling system are indirectly coupled to each other, there is the possibility of observing both fast and slow time scales.

To summarize, in this section we have shown that to reproduce the observed long transients we need at least a three component model. These long transients were observed in cells with positive values of $\frac{\lambda}{\sqrt{D}}$. The model shown in Equation (4.16) reproduces the observed transitions from finite to zero amplitude in a pulsatile manner. There are two possible mechanisms for recovering the amplitude after its transition, one is by the action of additive noise and the other by coupling Equation (4.16) with a slow inhibitor. The numerical solutions of Equation 4.16 coupled with a slow inhibitor are shown in Figure 4.16 a. It can be seen that the variable $v$ increases rapidly after stimulation inhibiting the oscillation amplitude, and after that $v$ stays at a finite value for some time and then it goes back to zero, then the oscillation amplitude is recovered. This would predict that there must be an important component inside the cell that once it becomes active the actin oscillations stop. But it was shown that another model can be constructed easily by using the components of Equations 4.16 and 4.18 and then doing a simple linear transformation. In this constructed model all of its components oscillate and show long transients, showing that in the experimental system a single time scale might not be associated with either the signaling cascade or the actin cytoskeleton.

\subsection{Chapter summary}

To study the biophysical properties of the observed periodic polymerization cycles in Dictyostelium discoideum, we have performed a series of experiments where we stimulated individual cells with a single pulse of cAMP. Since the control parameters that induces oscillations are not known, we have chosen cells randomly expecting that a small percentage would be in the oscillatory regime. The observed frequencies are below the resonance response of non-oscillatory cells when perturbed with periodic pulses of cAMP [114]. Also as noted in the Background chapter, these frequencies are one order of magnitude below the observed ones for the signaling system when it is decoupled from the actin cytoskeleton [102, 4, 48]. 


\subsection{CHAPTER SUMMARY}

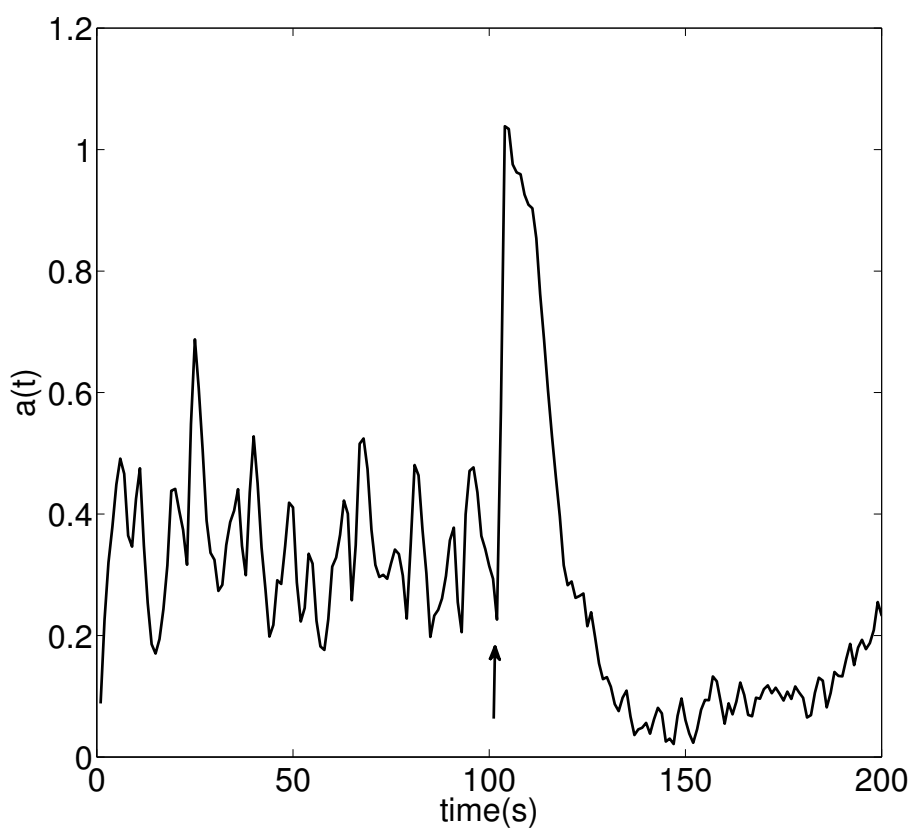

Figure 4.13: Amplitude obtained from a LimE-mRFP+Crn-GFP time series with high correlation time, the time of cAMP stimulation is denoted by a black arrow.
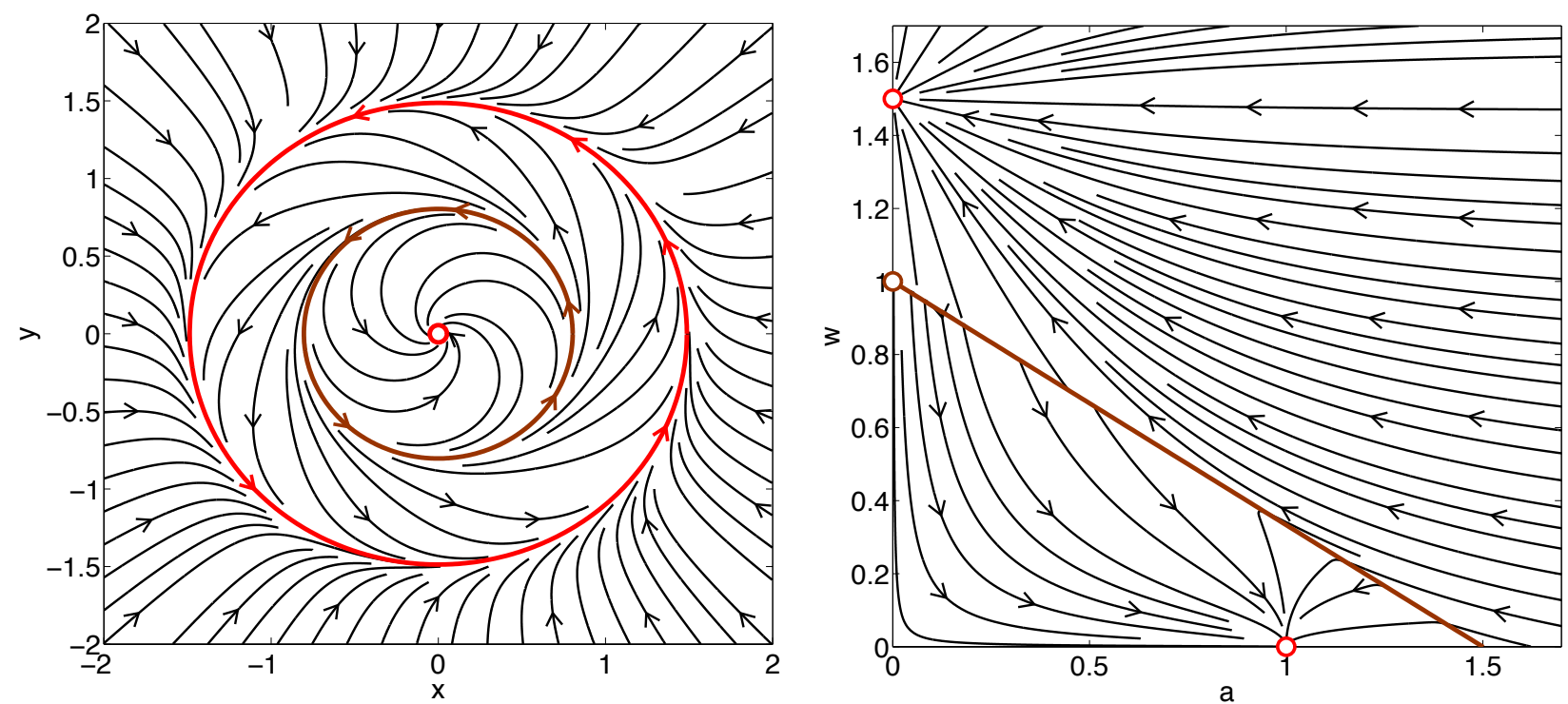

Figure 4.14: Phase portraits for Equations 4.13 (left) and 4.16 (right). 

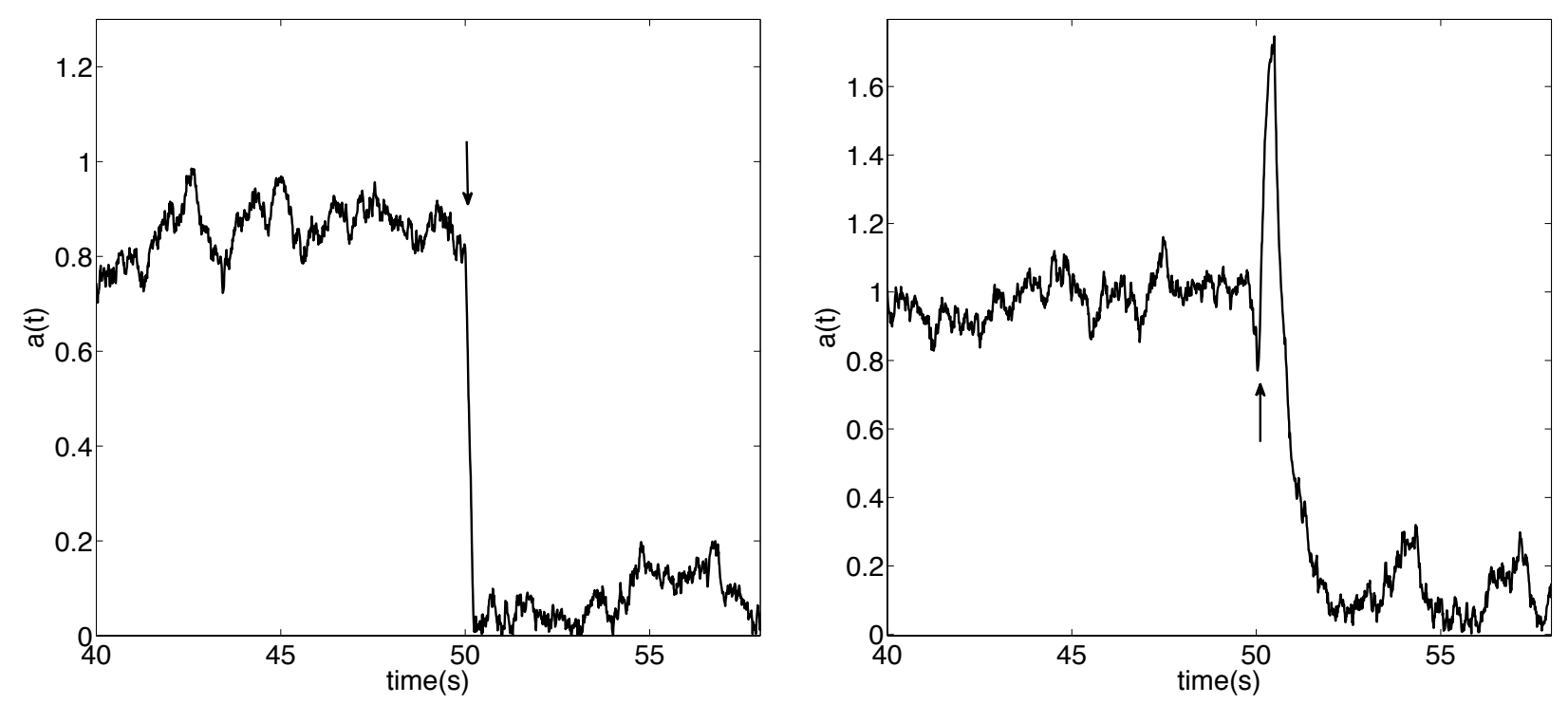

Figure 4.15: Numerical simulations of the amplitude dynamics for Equations 4.13 (left) and 4.16 (right).
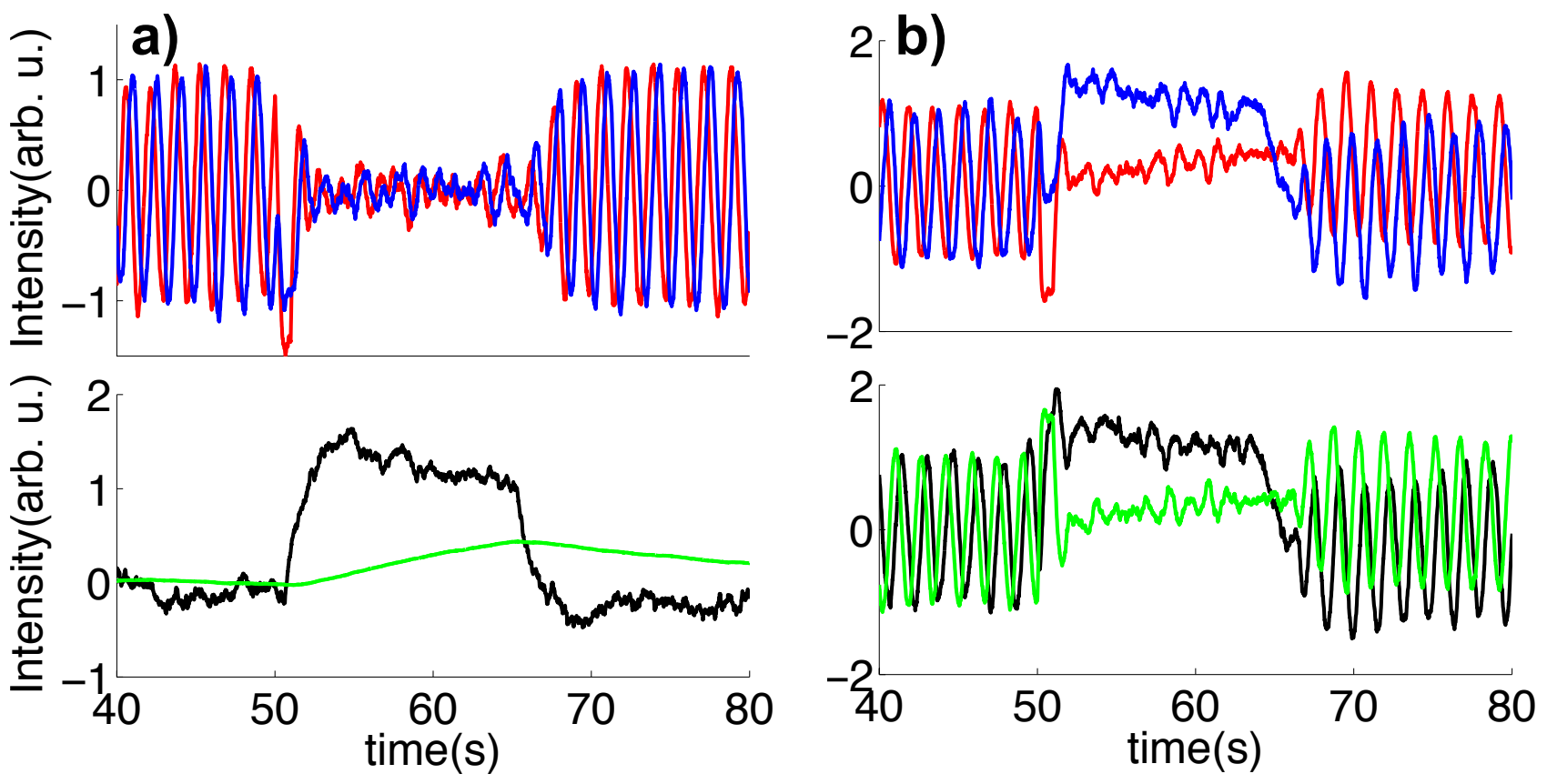

Figure 4.16: a) Numerical solution of Equation 4.16 where $x$ - red, $y$-blue, $w$-black and $v$-light green. b) Time series of the linear transformation $\overrightarrow{g^{\prime}}=\mathbf{M} \vec{g}$, where $\overrightarrow{g^{\prime}}=\left\{x^{\prime}-\right.$ red, $y^{\prime}-$ blue, $w^{\prime}-$ black, $v^{\prime}-$ light green $\}$. 


\subsection{CHAPTER SUMMARY}

Preliminary studies have shown that there is an integral relation between pseudopod extension and cytosolic LimE fluorescence. Meaning that when the cytosolic fluorescence depleted also a pseudopod is extended, but when the fluorescence recovers the pseudopod does not retract back. It is the hope that this result will help to understand subsequent studies on the control of pseudopod extension and its interactions with the signaling cascade.

A statistical analysis has been performed in the LimE time series which were classified as oscillatory. If the autocorrelation function of those time series had at least one oscillation period then it was defined as oscillatory. Each autocorrelation function is characterized by three parameters: frequency $\omega_{o}$, correlation time $\tau_{c}$ and variance $\sigma^{2}$. We interpreted these parameters by doing a systematic comparison of two possible scenarios with modelling. We have found that the majority of cells

are dominated by stochastic effects $\left(\frac{\lambda}{\sqrt{D}} \leq 1\right)$. In cells with self sustained actin oscillations we have found that the relative noise contribution is $35 \% \leq R N C \leq$ $100 \%$ when dominated by stochastic effects. We conjecture that D. discoideum developed a sensitivity to environmental noise in order to maintain high amplitudes at low metabolic costs.

When a cell is stimulated with cAMP it exhibits a transient cytosolic depletion of actin binding proteins. In cells characterized with positive $\frac{\lambda}{\sqrt{D}}$, we have seen an interplay between fast and low time scales in their transient response. These cells after being stimulated would halt their oscillatory activity completely, and then reestablish it after $\sim 100 \mathrm{~s}$. We have found the basic mathematical components needed to reproduce these transients. In the process we have realized that a two component model would not reproduce the observed responses. The addition of another variable would have two possible implications: a) a biological component quiescent during oscillatory activity destabilizes the amplitude when the cell is stimulated, or b) these time scales arise just by the mere distribution of the interaction parameters where all components are interconnected to each other.

All models presented in this Chapter are extensions of the Stuart-Landau oscillator. The fluctuations dependent effects and the cAMP induced long transients are independent from each other. Therefore we can write a generalized model describing our experimental observations. To construct it we need to take the additive noise from Equation (4.4), and the terms that induces amplitude transients 


\subsection{CHAPTER SUMMARY}

from Equation (4.16). The generalized model would have the following form,

$$
\begin{array}{r}
\frac{d z}{d t}=\left(\lambda(|z|, w)+i w_{o}-\epsilon|z|^{2}\right) z+\sqrt{D_{2}} \xi_{2}(t) \\
\frac{d w}{d t}=-\alpha w+\frac{\beta}{2}(1+\lambda(|z|, w)) \\
\lambda(|z|, w)=\tanh \left(\eta\left[w+\frac{w_{o}}{z_{o}}|z|-w_{o}\right]\right)
\end{array}
$$

but of course it is convenient to study both mechanisms separately.

Similar studies have been performed in Dictyostelium discoideum and other organisms. In mast cells antigen stimulation induces global oscillations and also traveling waves of actin binding proteins. It was found that in the case of global oscillations the calcium levels would also oscillate, but during actin traveling waves they were stable [120]. It would be interesting to see if a similar relationship holds in Dictyostelium discoideum, as it could elucidate details on the biochemical energy source behind these oscillations. Traction force microscopy measurements in Dictyostelium discoideum gives the traction energy exerted by a cell to the substrate [26]. The traction energy time profile is similar to that of a noise driven oscillator, it was found that the cell velocity and the local period (the time to made an oscillation cycle) have an inverse relation. The observed local periods range from 1 to 4 minutes, it would interesting to see if our observed fast oscillations also holds a relation with velocity.

To the knowledge of the author this is the first time that fluctuations in the actin cytoskeleton of Dictyostelium discoideum are studied formally. Fluctuations analysis has been used in other systems to study the cross correlation between ensembles of neurons [25], the connectivity of genetical networks [28, 31, 79], and the repair mechanism of the p53 system [41]. Studies on intrinsic and extrinsic noise has also been done in genetical networks [28, 31] with the aim to find their interconnectivity. So far the exact dynamical role of Coronin 1A and Aip1 in the cell cytoskeleton has not been addressed, in the next chapter we will analyze the responses of wild type cells along with genetical knockouts to confront this question. 


\section{Chapter 5}

\section{Dynamical responses of actin regulating proteins}

The actin cytoskeleton is a structure that it is constantly regulated by several actin binding proteins. Even when this structure is in its steady state, the interaction of the cytoskeleton with the abp's results in a continual polymerization and disassembly process in which the filaments have constant length on average. As we have seen in Chapter 4, Dictyostelium discoideum cells exhibit transient polymerized structures when stimulated with pulses of cAMP, and during this course its regulators, Coronin 1A and Aip1, also translocate to the cell cortex. In this chapter we will take advantage of this and study the dynamics of actin polymerization along with its regulators, Coronin $1 \mathrm{~A}$ and Aip1. The goal will be to infer their possible biological roles by studying their dynamical properties. These observations by themselves will not give us a complete picture of their regulatory role, therefore we will complement them by observing the polymerization dynamics, when either of these two regulators were genetically removed.

The first challenge that we face is the unknown distribution of internal parameters, as we have seen in Chapter 4, each cell has a different set of internal parameters (such as correlation time and variance). In the non oscillatory regime this distribution is reflected by the different physical properties of single responses such as the decay rate and frequency. The data by itself is noisy, as we have already seen fluctuations play an important role in the cell dynamics. Calculat- 


\subsection{ARTIFACTS FROM AVERAGED TIME SERIES}

ing ensemble averages of signals blindly to resolve the data might give misleading results, therefore we have developed a simple method to sort the data by their similarity in parameters. From the observed oscillatory properties of the induced actin polymerization, we know that the system is effectively behaving as a system regulated by a negative feedback loop.

In order to extract the possible effective feedback between LimE, Coronin 1A and Aip1, we have used a recently proposed method to transform the observed dynamics into symbols [81, 82. Finally we will see how the predictions obtained by the symbolic dynamics, differ from the response of genetical knockouts. For this it has been prove useful to characterize the signal curvatures at the extrema, since is a direct measure of the rate of change of the feedback at those times. This will provide us information on how the feedback has changed in the physiological responses between WT cells and knockouts. Finally we will give a heuristic interpretation of our results, which suggest that Aip1 and Coronin 1A inhibit a promoter of actin polymerization.

\subsection{Artifacts from averaged time series}

The recorded data from single cell experiments is noisy, and it is common practice to create an ensemble average from the recorded time series to resolve them. From these time series the experimenter will study the dynamical properties of the physical process, to possibly infer the mechanism behind it. As we have seen in Chapter 4, each cell will present different values of its internal parameters, up to the possibility of being in a different dynamical state to that of its neighbours. Therefore, averaging these time series might lead to misleading artifacts in the observed waveforms.

We can notice the possible artifacts that might appear by studying a simple thought experiment. Suppose that we record a signal $x(t)$ precisely at the time when the cell is stimulated. The response waveform consists of a sinusoidal with an exponential decay. The recording is corrupted by an additive stochastic term, and our observation can be expressed as

$$
x(t)=a_{o} \exp (-(\lambda-i \omega) t)+\xi(t)
$$




\subsection{ARTIFACTS FROM AVERAGED TIME SERIES}

where by definition, $\langle\xi(t)\rangle=0$. Then by doing an ensemble average from different measurements we would remove the effects of noise

$$
\langle x(t)\rangle=a_{o}\langle\exp (-(\lambda-i \omega) t)\rangle
$$

and keep an average of the deterministic part of the responses.

As different cells have different internal parameters, an isogenic population would be described by a parameter probability distribution $P(\lambda, \omega)$. The average response of the population would be

$$
\langle x(t)\rangle=a_{o} \int_{-\infty}^{\infty} \int_{-\infty}^{\infty} P(\lambda, \omega) \exp (-(\lambda-i \omega) t) \mathrm{d} \lambda \mathrm{d} \omega
$$

With this expression we can test the possible artifacts that might be given by different parameter distributions.

Then as a first case to study we define

$$
P(\lambda, \omega)=\delta(\lambda-\langle\lambda\rangle) \delta(\omega-\langle\omega\rangle)
$$

which correspond to a population with identical internal parameters. Using Equation (5.3) we calculate the observed averaged response function

$$
\langle x(t)\rangle=a_{o} \exp (-(\langle\lambda\rangle-i\langle\omega\rangle) t)
$$

In this case averaging removes the observed fluctuations and gives an exact representation of the response waveform. For this particular example we can extract significant parameters of the averaged signal and study its mechanism.

This case is unrealistic since we have observed already cells with different internal parameters in Chapter 4. To mimic these effects we can define

$$
P(\lambda, \omega)=\frac{\Theta(\lambda)}{\langle\lambda\rangle} \exp \left(-\frac{\lambda}{\langle\lambda\rangle}\right) \frac{1}{\sqrt{2 \pi \sigma_{\omega}^{2}}} \exp \left(-\frac{(\omega-\langle\omega\rangle)^{2}}{2 \sigma_{\omega}^{2}}\right)
$$

where $\Theta(\lambda)$ corresponds to the Heaviside function. The distribution of Equation (5.6) corresponds to an exponential and a Gaussian distribution for $\lambda$ and $\omega$ respectively. For this population the observed averaged response would be 


\subsection{DATA SEGREGATION FOR AVERAGING}

$$
\langle x(t)\rangle=a_{o} \frac{\exp \left(-2 \sigma_{\omega}^{2} t^{2}+2 i\langle\omega\rangle t\right)}{1+\langle\lambda\rangle t}
$$

We can notice three features from this result. First, there is a damping term that appeared due to the distribution for $\omega$, this term would appear even if the there was no damping term $\lambda$. This is due to the destructive interference that results from averaging different sinusoids of different frequencies. Second, the decay term due to $\lambda$ is not exponential anymore, so even in the absence of a sinusoidal part, the system would decay differently from how each cell actually does. Third, the response is going to oscillate with twice the average frequency from the distribution. Therefore the observed average will differ substantially from a single cell response since it depends on the parametric distribution.

In experiment we do not know neither the expression of the response waveform, nor the parameter distribution. Therefore it is not possible to know beforehand how severe will be the effects of the parametric distribution, and how much the observed waveform will deviate from the deterministic part of a single cell response. Therefore in this work we will study the response from single cells, but since fluctuations are present, it its also necessary to do averaging. In the next section we will describe the algorithm to systematically select similar waveforms for averaging. This will avoid the arbitrary selection that an experimentalist does between "responsive" and "non responsive" cells, and also to reject measurements that consist in pure noise drift.

\subsection{Data segregation for averaging}

As mentioned in the last section and investigated experimentally in [92, 111], isogenic Dictyostelium discoideum cells respond differently to chemoattractant stimulation due to its natural cell to cell variability. But the intrinsic cell to cell variability will induce new artifacts which cannot be eliminated by simple averaging. With this problem in mind the following question arose: How to group cells with similar internal parameters for averaging the time series?

Using simple concepts of dynamical systems we developed a simple algorithm. Suppose that our system has its internal parameters constant during our observa- 


\subsection{DATA SEGREGATION FOR AVERAGING}

tion time. Such system can be defined by a set of deterministic rules given by a differential equation of the form

$$
\frac{d \vec{x}}{d t}=f(a, \vec{x})
$$

where $a$ is the set of internal parameters and $\vec{x}$ is the state of our system. Equation (5.8) will have a unique solution for any given initial condition $\vec{x}_{o}$, given that $f(a, \vec{x})$ is Lipschitz continuous [44]. All possible solutions of Equation (5.8) can be mapped into a vector field called the phase portrait [98]. For a given initial condition the system will evolve on a specific trajectory of this phase portrait, experimentally this initial condition would be given by disturbing the steady state of the cell with a pulse of cAMP. Since some of our cells have two labels present, these can be used to map a two dimensional phase portrait. If two cells have similar internal parameters, its response must be marked by a similar trajectory in their phase portrait, and in the same way, for cells differing by much in its internal parameters the trajectories will deviate by a large amount.

Figure 5.1 shows an example of different realizations for a simple two variable system, $\{x, y\}$, with a decay rate $\lambda$ and a frequency $\omega$. The time series shown in panels 1,2,3 \& 4 have different parameters (details of model and simulations are given in Appendix B). The trajectories in the phase portrait are also shown and are numbered according to its corresponding panel. It can be seen that some trajectories have similar paths, while the difference is higher for others. Therefore to quantify the similarity between parameters, the following measure between a pair of trajectories is used

$$
\left\langle d(x, y)_{i, j}\right\rangle=\left\langle\sqrt{\left(x_{j}(t)-x_{i}(t)\right)^{2}+\left(y_{j}(t)-y_{i}(t)\right)^{2}}\right\rangle<\epsilon
$$

where $\epsilon$ is a threshold value that defines the maximum value that two trajectories can differ. If two trajectories have a distance below $\epsilon$, then they have similar internal parameters and belong to the same group. In the phase portrait of Figure 5.1 it can be seen that trajectory 4 is very close to trajectory $3\left(\left\langle d_{34}\right\rangle=0.1546\right)$ compared to its distance with $1\left(\left\langle d_{14}\right\rangle=0.3580\right)$ and $2\left(\left\langle d_{24}\right\rangle=0.4374\right)$. This is because in the performed simulations the parameters for the time series in panels 4 and 3 do not differ by much (details are given in Appendix B). 


\subsection{DATA SEGREGATION FOR AVERAGING}

Figure 5.2 shows the phase space distance $d_{i j}$ between a single trajectory $\left\{x_{j}, y_{j}\right\}$ and a set of trajectories $\left\{x_{i}, y_{i}\right\}$. For these simulations the values of $\lambda$ and $\omega$ were varied for $\left\{x_{i}, y_{i}\right\}$ and kept constant for $\left\{x_{j}, y_{j}\right\}$ to $\lambda_{o}$ and $\omega_{o}$ (details in Appendix B). In the set of curves in Figure 5.2 it can be seen that they have a general minimum at around $\lambda_{o}$. Deviating the values of $\omega$ also increases the value of $d_{i j}$, where in Figure 5.2 it can be seen that the minimum is also around $\omega_{o}$. Further it can be noted that this measure is very sensitive to changes in $\omega$ for some values of $\lambda$.

Therefore in practice we did the following, for each cell line the value of $\epsilon$ was set to a constant value. The phase space distance was determined for each of the measured time series with respect to the others. For each cell it was found the amount of matching trajectories that fulfilled the condition $\left\langle d_{i, j}\right\rangle<\epsilon$, then they were grouped and the arrays with the highest number of matches were taken for analysis. Since the selection of the value $\epsilon$ will be arbitrary, we cannot get away completely from the effects of parameter distribution, the main success of the algorithm was to take out time series that show a substantial amount of noise drift. The drifting time series contribute to the time dependence of the error bar, and therefore the physical process would appear as non stationary [15].

In Figure 5.3 we have a comparison of the total recorded LimE time series before implementation of the algorithm (a) and after implementation (b). In these time series the second label was Coronin 1A, so the measured phase portrait trajectory distance is in the LimE and Coronin 1A space. We can notice the differences of the error bars in both panels, if we do not implement the proposed algorithm the standard deviation of the time series increases over time (Figure $5.3 \mathrm{a}$ ). This is a signature of the non-stationarity of some of the series which do not contain a constant baseline [15]. After implementing the algorithm, with $\epsilon=0.25$, we can see that the standard deviation drift is reduced dramatically (Figure $5.3 \mathrm{~b}$ ). The base line at the beginning and at the end of the time series are not the same, this can be attributed to fluorophore photobleaching [62], which decreases the fluorescence intensity when exposed to the excitation laser. In the case of knock out cell lines in which we can only visualize LimE, but this algorithm can also be implemented even though Equation (5.9) is less restrictive. A test as the one shown in Figure 5.2 had shown that the results are qualitatively the same if we 


\subsection{WILD TYPE CELLS}

have only one fluorescent label and using $\left\langle d(x)_{i, j}\right\rangle=\left\langle\sqrt{\left(x_{j}(t)-x_{i}(t)\right)^{2}}\right\rangle<\epsilon$.
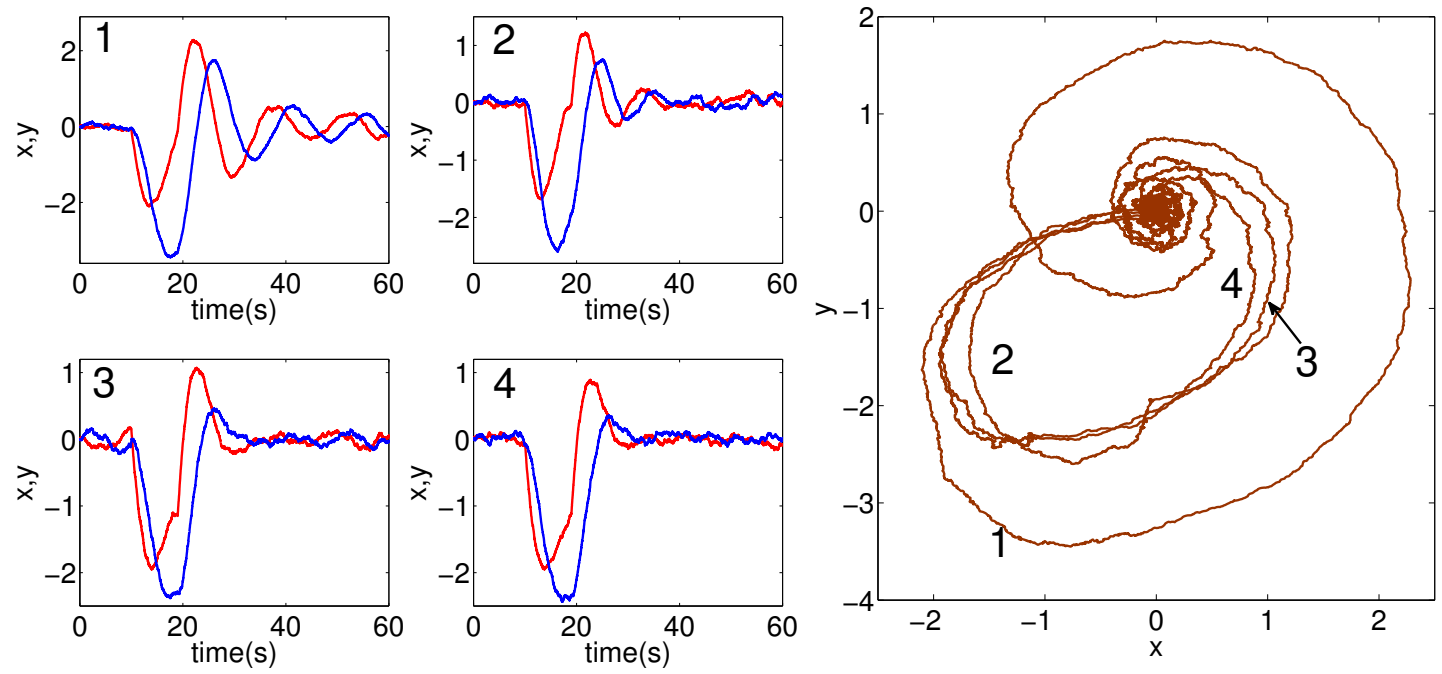

Figure 5.1: Different realizations for a two variable model, x-red and y-blue. Each panel, 1-4, corresponds to time series with different parameters $\lambda$ and $\omega$. On the right side plot is the phase space trajectories for each panel is shown. Due to the difference in parameters the average distance between trajectories are different.

\subsection{Wild type cells}

As mentioned in chapter 2, we subjected our cells to an external flow of BCMCMcAMP (parameters given in the Materials and Methods section). The recording time was set to one minute for all cell lines, except for Aip1 $\Delta$ cells which was set to $100 \mathrm{~s}$. After $7 \mathrm{~s}$ of recording, a one second pulse of cAMP was administered to the cells and the consequential cytosolic fluorescence depletion was quantified. In Figure 5.4 we have the time evolution of a LimE-mRFP+Coronin 1A-GFP cell, the white spot in the picture sequence corresponds to the time of cAMP uncaging. At $14 s$ we can see that the LimE label (red) has translocated to the cell cortex, as it can be noticed by the darkening of the cytosol and the increase in brightness at the cortex. During this time frame the cytosolic fluorescence for Coronin 1A (green) is practically unchanged, but later at $t=18 \mathrm{~s}$, the cytosol becomes darker for the 


\subsection{WILD TYPE CELLS}

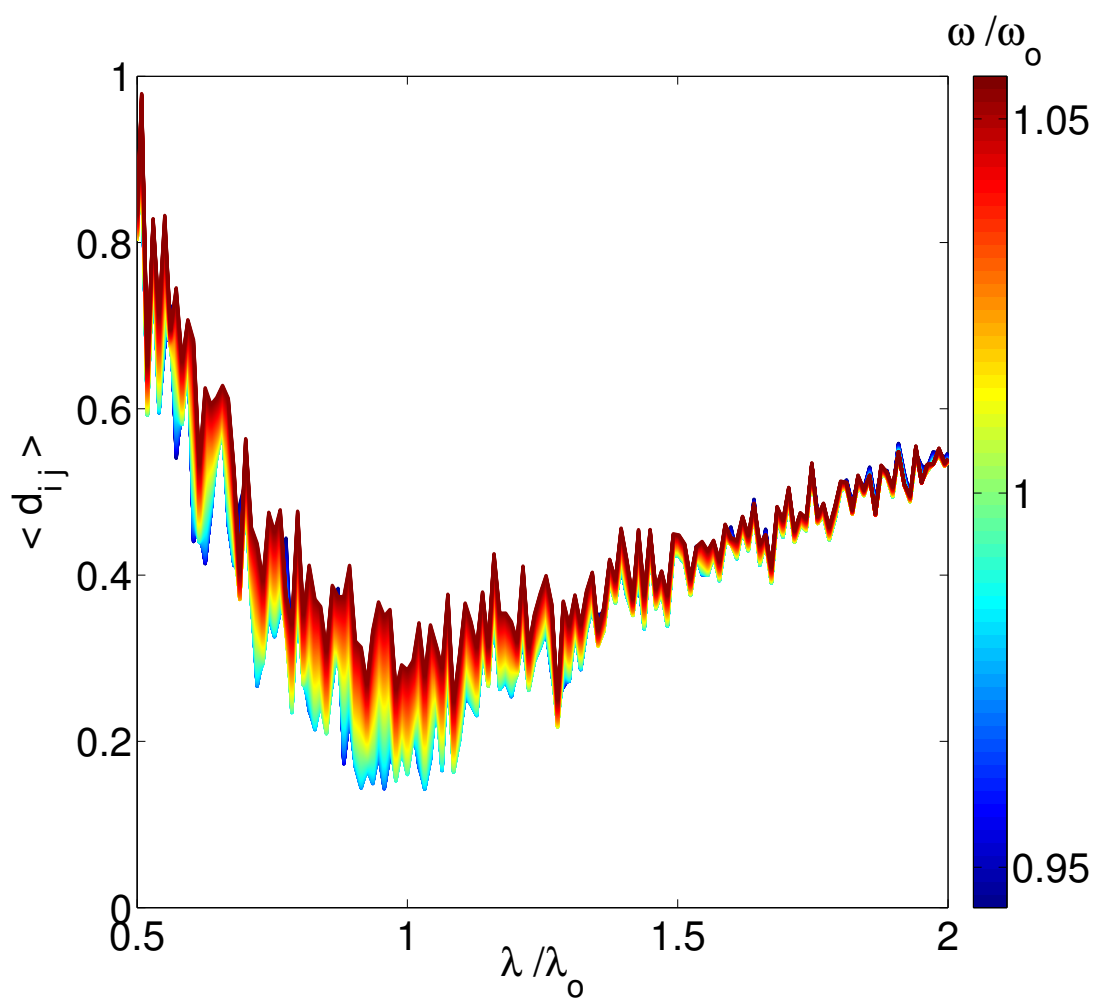

Figure 5.2: Phase space trajectory distance between trajectories created by simulation. One trajectory $\left\{x_{j}(t), y_{i}(t)\right\}$ was kept with constant parameters $\lambda_{o}$ and $\omega_{o}$, while for trajectories $\left\{x_{i}(t), y_{i}(t)\right\}$ its parameters $\lambda$ and $\omega$ were varied. We can notice that a general minima is situated around $\lambda_{o}$ and $\omega_{o}$

green label and also the cortex brighter. This shows that there is a delay between the functional response of LimE and Coronin 1A. At $27 s$ both labels appear to be recovered, but an analysis from the extracted time series shows details that cannot be discerned just by looking at the picture sequence (Figure 5.6). A similar progression is shown in Figure 5.5 for a LimE-mRFP+Aip1-GFP cell, though the cytosolic depletion of Aip1 is not discernible by naked eye. The measurements of average cytosolic fluorescence will show again details of the functional response of Aip1.

In Figure 5.6 a, we have the average cytosolic response for LimE-mRFP (red line, $n=31 / 59$ cells), Aip1-GFP (black line, $n=16 / 35$ cells) and Coronin 1A- 


\subsection{WILD TYPE CELLS}
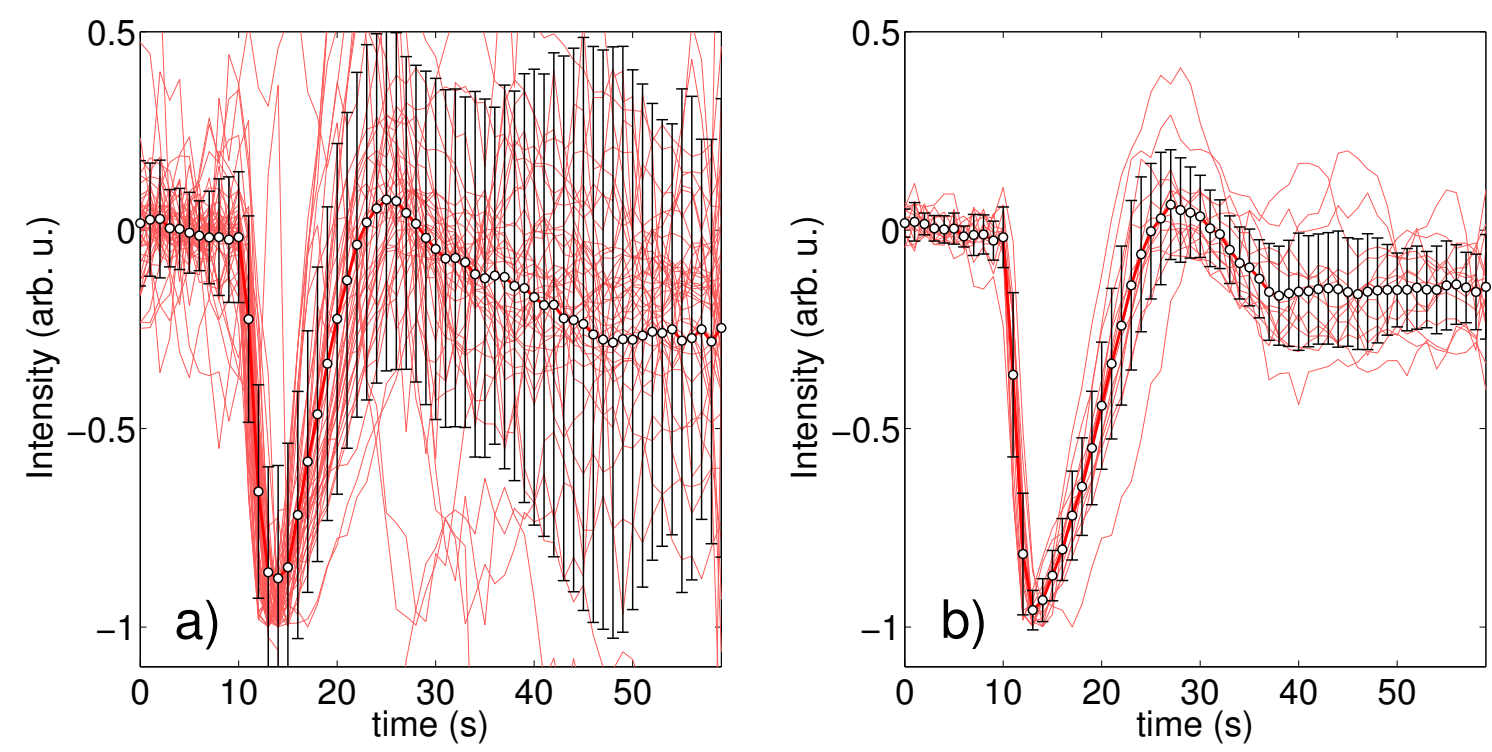

Figure 5.3: Recorded LimE time series for a LimE-mRFP+Crn-GFP cell. a) All the time series recorded for this particular cell line. b) The remaining time series after implementing the Equation $(5.9)$ with $\epsilon=0.25$

GFP (blue line, $n=15 / 24$ cells). As in the previous chapter, all the raw signals had their mean of the first seven seconds subtracted and were normalized to their minima, which is found by fitting a parabola to the lowest measured point along with its preceding and succeeding points. To segregate the time series we set the threshold value of Equation $(5.9)$ to $\epsilon=0.25$. In the figure the time when the cells are stimulated is denoted by a black arrow. Figure 5.6 b, c, \& d, shows the first $17 s$ for each protein with their respective error bars.

The response of LimE consists of a sudden fluorescence decrease after stimulation, followed by an overshoot and then relaxing back to equilibrium. The response for Coronin 1A and Aip1 are qualitatively the same as the one for LimE, except that they show a delay, consistent with our previous observations in Chapter 4 . The polymerization process can be easily interpreted by comparing the LimE picture sequence (Figures $5.4 \& 5.5$, red label) along with its time series (Figure 5.6, red curve). When the cytosolic fluorescence depletes, the time series drop to the minimum, and at the same time a fluorescent ring is created at the cell cortex. This means that during this time actin is being polymerized at the cell cortex, and 


\subsection{WILD TYPE CELLS}

in the same way when the fluorescence is recovering the actin cortex is being depolymerized. Therefore the observed overshoot can be understood as a reduction of filamentous actin at the cortex and the consequential relaxation as its recovery.

The response time for LimE-mRFP can be estimated by looking at the time when the signal deviates from its base line (Figure 5.6b). The standard deviation has an average value of 0.035 before applying stimulation, therefore a significant deviation must be higher than that value. The first significant deviation is observed at $t=11 \mathrm{~s}$, where the fluorescence value is $-0.47 \pm .23$. Then we can conclude that LimE starts translocating at around $3 s \leq t \leq 4 s$ after stimulation. For Aip1GFP the values around the baseline are fluctuating strongly. But still the response time can be estimated, after one second of stimulation the signal for Aip1 decays monotonically (Figure 5.6.). A close inspection shows that before stimulation the average standard deviation is 0.097, and the first significant deviation is at $9 \mathrm{~s}$ with a fluorescence value of $-0.10 \pm .11$. The line continues to decay monotonically with only one point being an exception at $t=11 \mathrm{~s}$, suggesting that this could be related to the activation of LimE. The translocation time for Aip1-GFP is between $2<t<3 s$ after stimulation, ahead of that of LimE, which suggest that Aip1 displaces before actin polymerizes.

Finally the same analysis for Coronin 1A shows an average standard deviation of 0.077 , before stimulation, and at $12 s$ the fluorescence is $-0.19 \pm 0.13$, therefore it translocates between $4<t<5 s$ after stimulation. Therefore these proteins are activated in sequential order, Aip $1 \rightarrow \operatorname{LimE} \rightarrow$ Coronin $1 A$. But the arrival of the three signals to their minima is not in the same order (analyzed in detail in the section 5.3.2). Also it can be seen that there is a strong correlation between Coronin $1 \mathrm{~A}$ and Aip1 specially during their minima.

\subsubsection{Cell to cell variability in WT}

Here we are going to analize the different responses that we have obtained by implementing the algorithm proposed in this chapter. Figure 5.7 shows the different responses sorted for wild type cells. The difference in internal parameters will be reflected in different characteristics of the response waveform, such as decay rate and frequency, to mention some. In panel a) we see time series with a similar LimE 


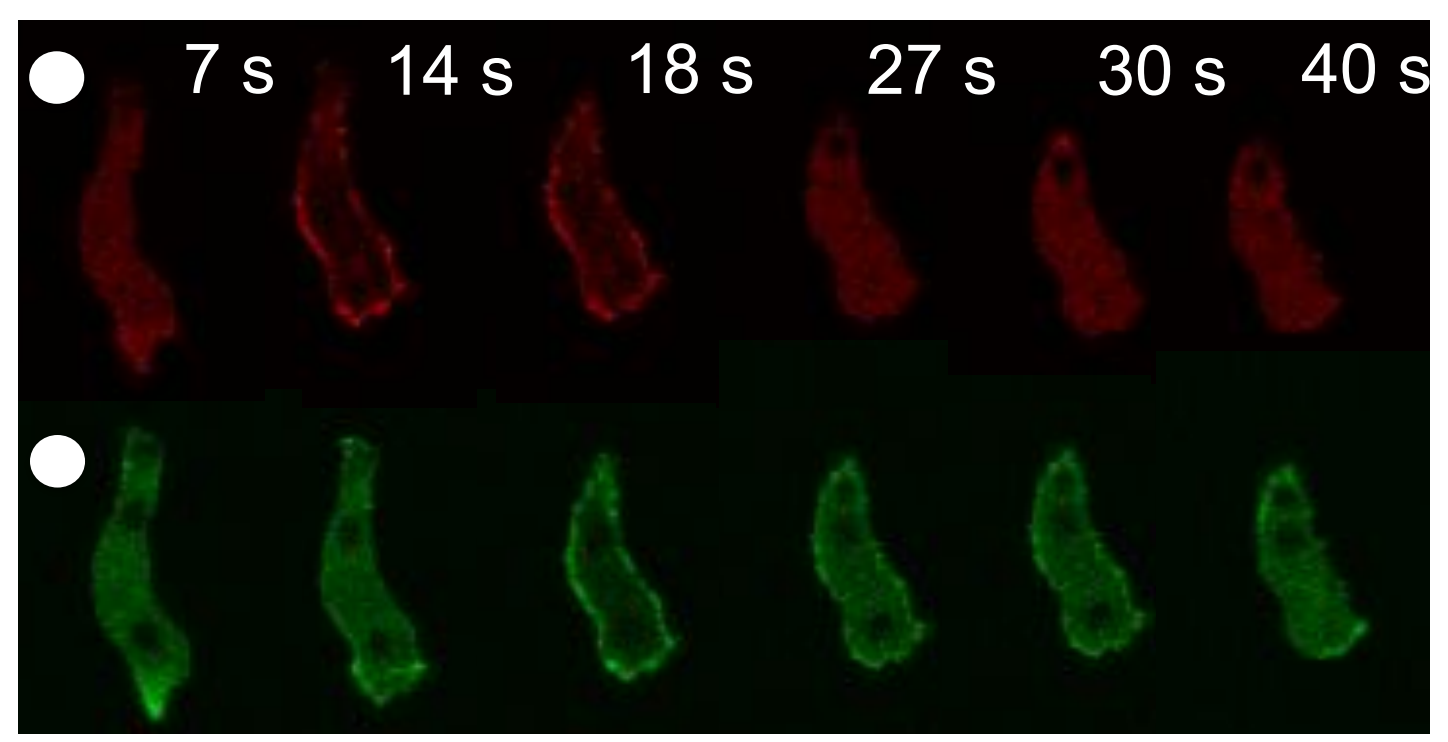

Figure 5.4: Time sequence of a double labeled cell with LimE (red) and Coronin $1 \mathrm{~A}$ (green). At $7 s$ a pulse of cAMP is released and consequently the proteins transiently translocate, as can be seen by the darkening of the cytosol.

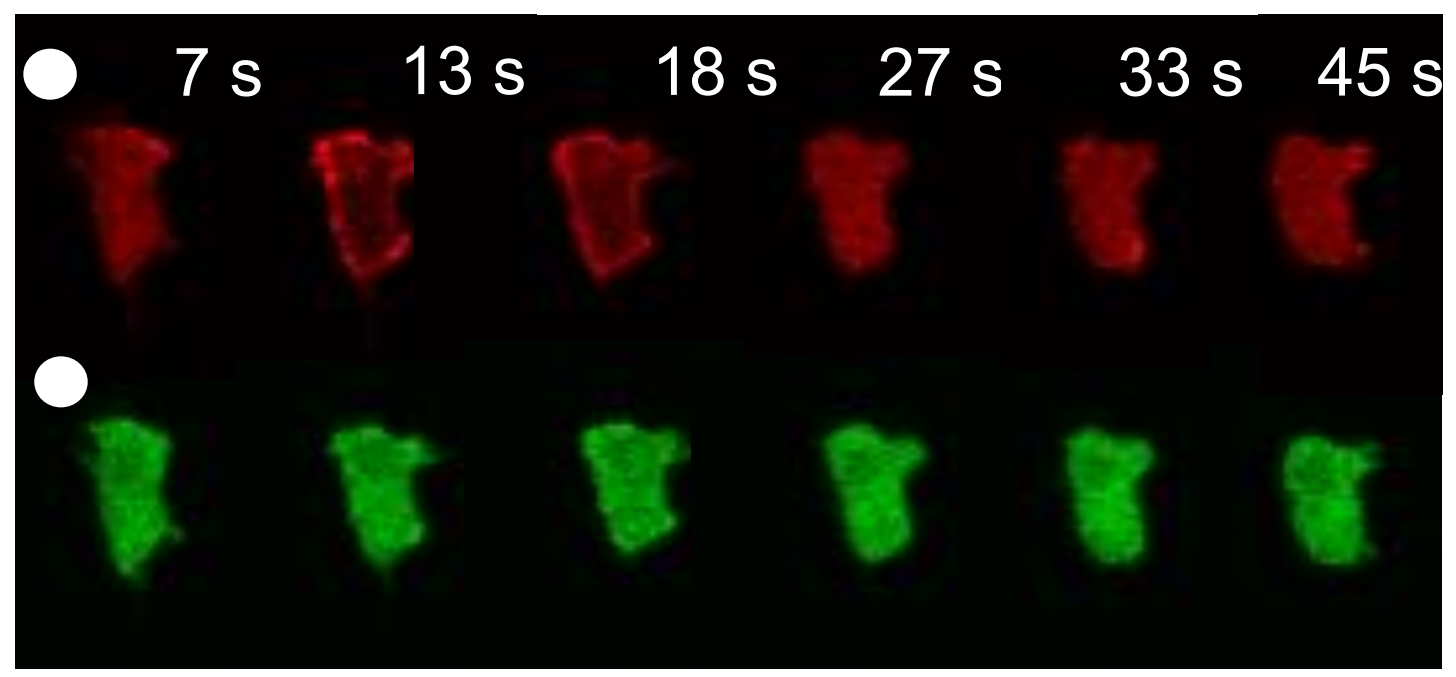

Figure 5.5: Time sequence of a double labeled cell with LimE (red) and Aip1(green). At $7 s$ a pulse of cAMP is released and consequently the proteins transiently translocate, as can be seen by the darkening of the cytosol. 


\subsection{WILD TYPE CELLS}

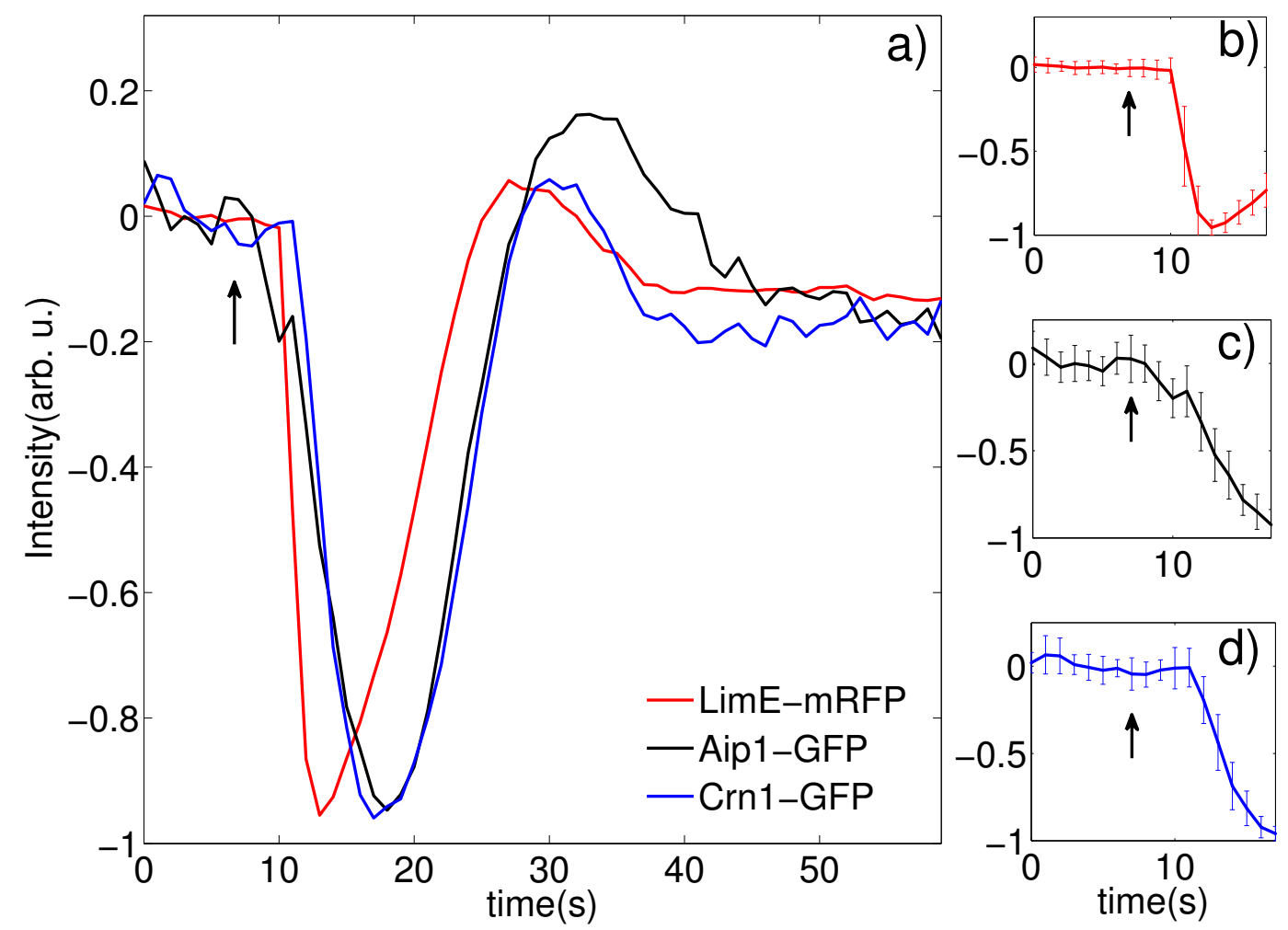

Figure 5.6: Time evolution of the mean cytosolic fluorescence. a) Averaged time series for LimE (red), Coronin 1A (blue) and Aip1 (black). b), c) and d) show the first $15 s$ of the time series with their corresponding errorbars for LimE, Aip1 and Coronin 1A respectively. These were used to quantify the time for initiation of the translocation. 


\subsection{WILD TYPE CELLS}

response for cells either co-expressing Coronin 1A-GFP or Aip1-GFP. The LimE signals differ in the way they decay towards equilibrium, after the overshoot the decay is slower compared to the LimE signal shown in Figure 5.6. It can be seen that the series have such a degree of similarity that they overlap on top of each other, except for their individual fluctuations. The co-expressed labels also present a similar decay rate, though the LimE signal in panel a) differ in their turnover at the minima between cells co-expressing Coronin 1A or Aip1. This turnover is slower for cells co-expressing Aip1 than for the ones with Coronin 1A. So although these signals present some degree of similarity, the difference in turnover shows that they have also different internal parameters.

In panel b) for both Coronin 1A and Aip1, the LimE signal keeps oscillating for at least two cycles after the first overshoot, it is interesting that Coronin $1 \mathrm{~A}$ has a lower baseline after the overshoot and no apparent signs of ringing. Which is the opposite for Aip1, that shows a higher baseline after the overshoot, but also it has no discernible signs of ringing. In case c) we have cells which exhibited a higher overshoot in the three labels compared to the series of Figure 5.6. In case d) for LimE-mRFP+Aip1-GFP, the signals showed a pulse response with no detectable overshoot, which may indicate that in these cells there is a very weak feedback between its components. Case d) for LimE-mRFP+Coronin 1A-GFP shows a marginal case with very strong overshoot and weak damping. In this section get an insight of the different responses that can be detected from different cells, but also it has shown that the response segregation algorithm proposed has been successful in sorting.

\subsubsection{Symbolic dynamics}

The timing of translocations to the cell cortex and their oscillatory properties, suggest that there is a causal relation between LimE, Coronin 1A and Aip1. To uncover these possible indirect interactions, we have used a method proposed to transform the observed dynamics into a symbolic sequence [81, 82]. With this sequence it is possible to uncover the possible effective feedback loop, which gives information on the activating and inhibitory interactions between components. To transform the observed signals into symbols, the following algorithm has to be 


\subsection{WILD TYPE CELLS}
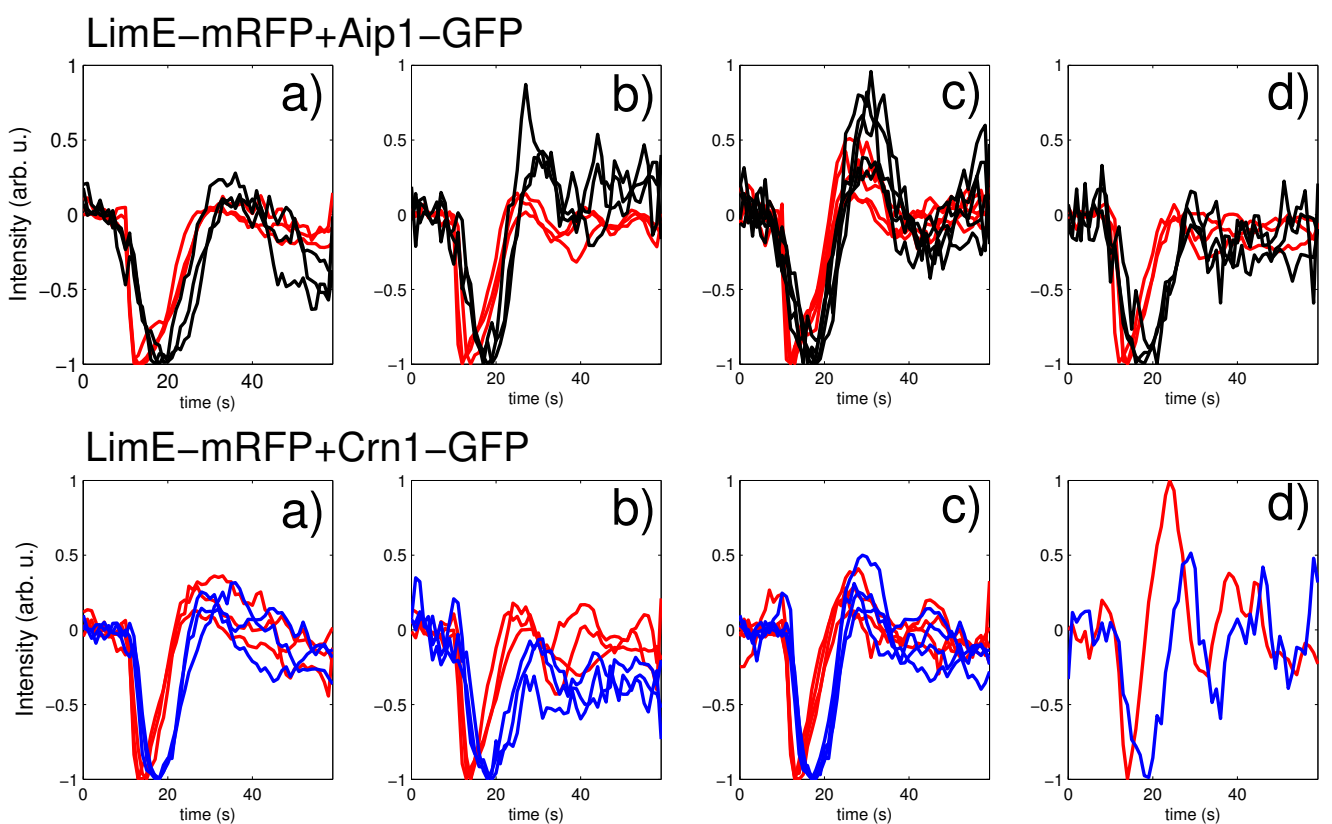

Figure 5.7: Different types of responses obtained in WT cells by applying the algorithm mentioned in section 5.2. It is evident that these cells have different internal parameters as they present different overshoots and ringing. 


\subsection{WILD TYPE CELLS}

followed [81]:

- Localize each extrema in the observed signals to divide it into different segments. Each segment is bounded by the extremum of one signal and by the next observed extremum from another signal, therefore it is necessary that the observed extrema is sequential. In our case it would be the extrema of the LimE, Aip1 and Coronin 1A time series.

- Once the signals are segmented, assign the symbols " + to signals with positive slopes and "-" to signals with negative slopes.

- Look at the sequential changes of the symbolic progression to infer the interactions of each segment. For example if we measure two species, $\{A, B\}$, and find the following sequence $\{+,+\} \rightarrow\{-,+\} \rightarrow\{-,-\} \rightarrow\{+,-\} \rightarrow$ $\{+,+\}$, we can infer their interaction by making the following observations. In the first transition $B$ stayed with a positive slope while $A$ changed to a negative one, then from that segment it can be inferred that $B$ inhibits $A$. This is since the positive increase of $B$ slows down $A$, up to the point of changing its slope. In the same way in the second transition, $A$ activates $B$, since the decreasing values of $A$ de accelerates $B$ into the direction of its decrease.

This method is justified only if the interactions between elements are monotonous, meaning that activators always work as activators and inhibitors always work as inhibitors. A technical explanation of this method is based on the division of the phase portrait by the nullclines into different sections. These are the regions in the phase portrait where the time derivative of a component is zero, once a phase space trajectory crosses a nullcline [81], one of the components will show an extrema. The symbols are representing the transition to different sections of the phase portrait [42, 118].

Therefore the first step to apply this method consists in localizing the extrema observed in the LimE, Coronin 1A and Aip1 signals. For each measured signal, included in the averaging of Figure 5.6, we have localized their extrema. To obtain these parameters we fitted a fourth order polynomial to the LimE signal for the range $13 s \leq t \leq 22 s$ and also for Aip1 and Coronin 1A at the ranges $15 s \leq$ 


\subsection{KNOCKOUT CELLS}

$t \leq 26 s$ and $14 s \leq t \leq 26 s$ respectively. The curves were fitted by minimization of least squares and with the obtained algebraic expression we have localized the minima. Figure 5.8 (top left) shows an overlay of the obtained polynomials along with their mean values for the minima, showing error bars for intensity and time. The times where the minima are reached are $\tau_{\text {LimE }}^{\min }=6.30 \pm 0.71 \mathrm{~s}$ for LimE, $\tau_{A i p 1}^{\min }=11.04 \pm 0.90 \mathrm{~s}$ for Aip1 and $\tau_{\text {Coronin } 1 A}^{\min }=10.70 \pm 1.25 \mathrm{~s}$ for Coronin. Using the same procedure we obtained the times of the maxima, except that this time we fitted a third order polynomial to the Aip1 and Coronin 1A signals. We used the ranges $23 s \leq t \leq 46 \mathrm{~s}$ for LimE, $25 \mathrm{~s} \leq t \leq 40 \mathrm{~s}$ for Coronin $1 \mathrm{~A}$ and $25 s \leq t \leq 47 s$ for Aip1, the found values for the maxima after stimulation were $\tau_{\text {LimE }}^{\max }=21.85 \pm 2.23 \mathrm{~s}, \tau_{\text {Coronin } 1 A}^{\max }=23.66 \pm 1.84 \mathrm{~s}$ and $\tau_{\text {Aip } 1}^{\max }=26.06 \pm 3.11 \mathrm{~s}$.

This information along with the slopes of the signal is enough to reconstruct the given symbolic dynamics, in Figure 5.9 (left) we have the reconstructed dynamics. In the first transition LimE changes slope while Coronin 1A and Aip1 stay with the same one, this suggest an indirect inhibition of LimE by either Coronin 1A or Aip1. In the second transition Coronin 1A changes slope to the same as LimE and in the third Aip1 changes to a positive slope. After that LimE has a negative slope then is followed by Coronin 1A and finally Aip1 also changes. Therefore the suggested loop is LimE activates Coronin 1A, Coronin 1A activates Aip1 and Aip1 inhibits LimE. In Figure 5.9 (right) we have the inferred interaction topology, along with the possible links that cannot be distinguished by this method (denoted by red links). As mentioned in the previous section, when the time series of LimE has a negative slope the cell is effectively depolymerizing and polymerizing when positive. The graph in Figure 5.9 gives the following prediction: In the absence of Coronin $1 A$ or Aip1, the polymerization and depolymerization turnover rates will be slowed down. Now we can proceed to analyze the data from the knockouts and determine how this picture deviates from the actual experimental responses.

\subsection{Knockout cells}

To observe if there is any causal relation between LimE, Coronin 1A and Aip1, we performed the same experiments with their respective genetical knockouts. In these cells the signals measured was LimE, in the cell lines LimE-GFP+Aip1 $\Delta$ 


\subsection{KNOCKOUT CELLS}
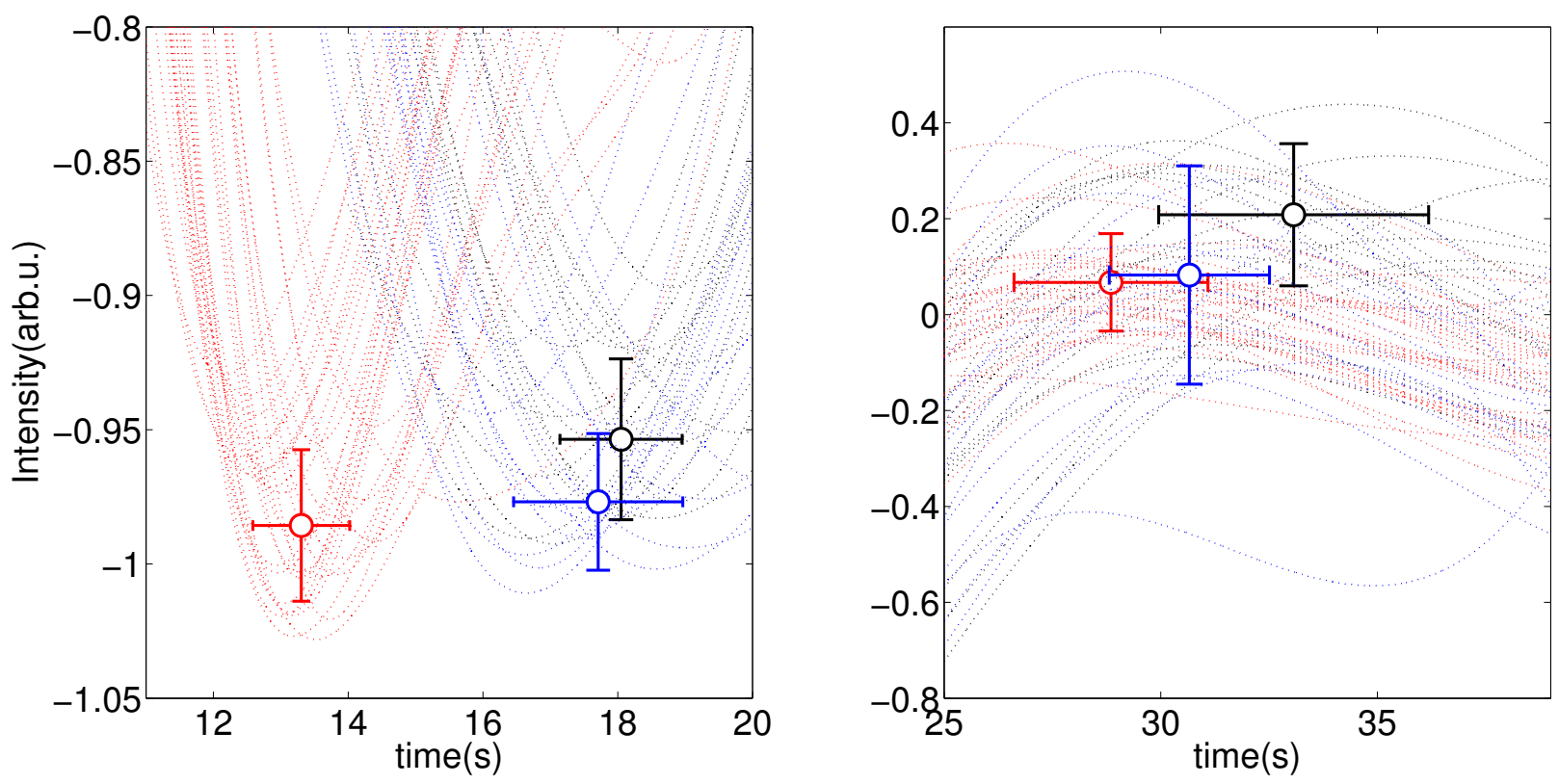

Figure 5.8: Time to reach the minimum (left) and the maximum (right) in the responses of LimE(red), Coronin $1 \mathrm{~A}($ blue) and Aip1(black) in WT cells.

and LimE-GFP+Coronin $1 \mathrm{~A} \Delta$. In general the variability of responses in knockout cells is higher than in the wild type. In Figure 5.10 a, we have the time evolution for an ensemble average measured for 11/19 cells from the LimE-GFP+Aip1 $\Delta$ cell line and 10/23 cells from the LimE-GFP+Coronin 1A $\Delta$ cell line (LimE wild type is the same as in the previous section). It is obvious that there are immediate consequences on the removal of these two proteins, proving that these are of high relevance for the polymerization dynamics.

As in the previous section we start by analyzing the activation times, in Figure 5.10 c we can see clearly that the activation time for the Aip1 $\Delta$ is identical to the one of the wild type within the limits of experimental resolution. The average standard deviation before stimulation is 0.064 , at $t=11 \mathrm{~s}$ the fluorescence value is $-.30 \pm .2094$ which is a significant deviation from the base line.Though for the Coronin $1 \mathrm{~A} \Delta$ cell the activation time appears to be between $2<t<3 s$ after stimulation, since a significant deviation since the fluorescence values at $10 s$ is $-0.13 \pm .1215$ and the average standard deviation before measurement is 0.081 . The change of the activation time for LimE in Coronin $1 \mathrm{~A} \Delta$ cells opens the follow- 


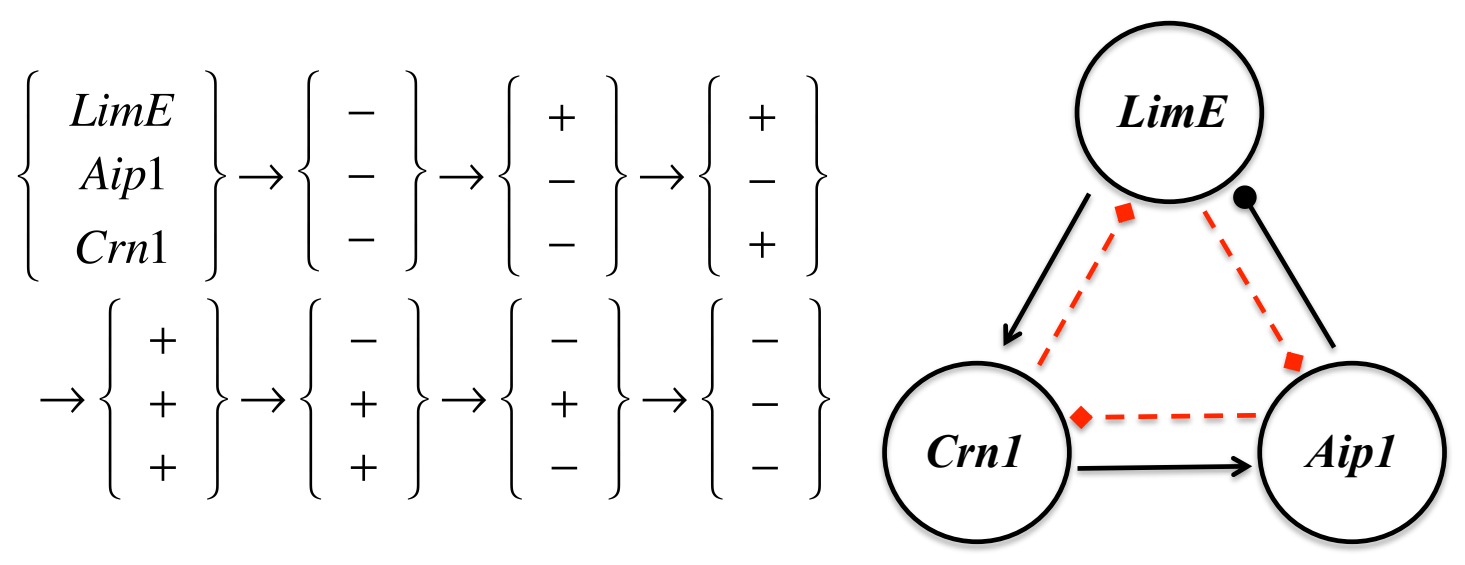

Figure 5.9: Symbolic dynamics obtained by the algorithm proposed in [81, 82]. Left, the evolution of the symbolic dynamics for LimE, Coronin 1A and Aip1 for WT cells. Right, the inferred interaction graph with excitatory links (pointed arrows), inhibitory links (ball arrows) and unknown links (red).

ing question: is the response of LimE faster due to a change in the dynamics of the cytoskeleton or due to changes in the signaling system? It has been observed that Coronin 1A not only has functions in the cytoskeleton but also in development [110]. In the following sections we will analyze in more detail the single cell responses and interpret the data with some tools developed to interpret biochemical networks.

\subsubsection{Cell to cell variability in Knockouts}

Figure 5.11 shows the variability of responses for knockout cells. A first look into the figure, we can see that Aip1 $\Delta$ has a higher variability on the response waveforms since they differ substantially from each other. For example in panel a, we observe a response with a sharp turnover at the minimum and a sharp overshoot, indicating fast depolymerization and polymerization respectively. The response shown in panel $b$ is considerably different from the response in a. This waveform has interesting features consequence of the absence of Aip1, such as a very slow turnover at the minima, where the signal maintains this level for roughly $10 \mathrm{~s}$. Therefore during this period the actin filaments that were polymerized at the cell cortex are stable for reasonably long time. After that these filaments depolymerize and repolymerize fast as can be seen by the sharp overshoot. In 


\subsection{KNOCKOUT CELLS}
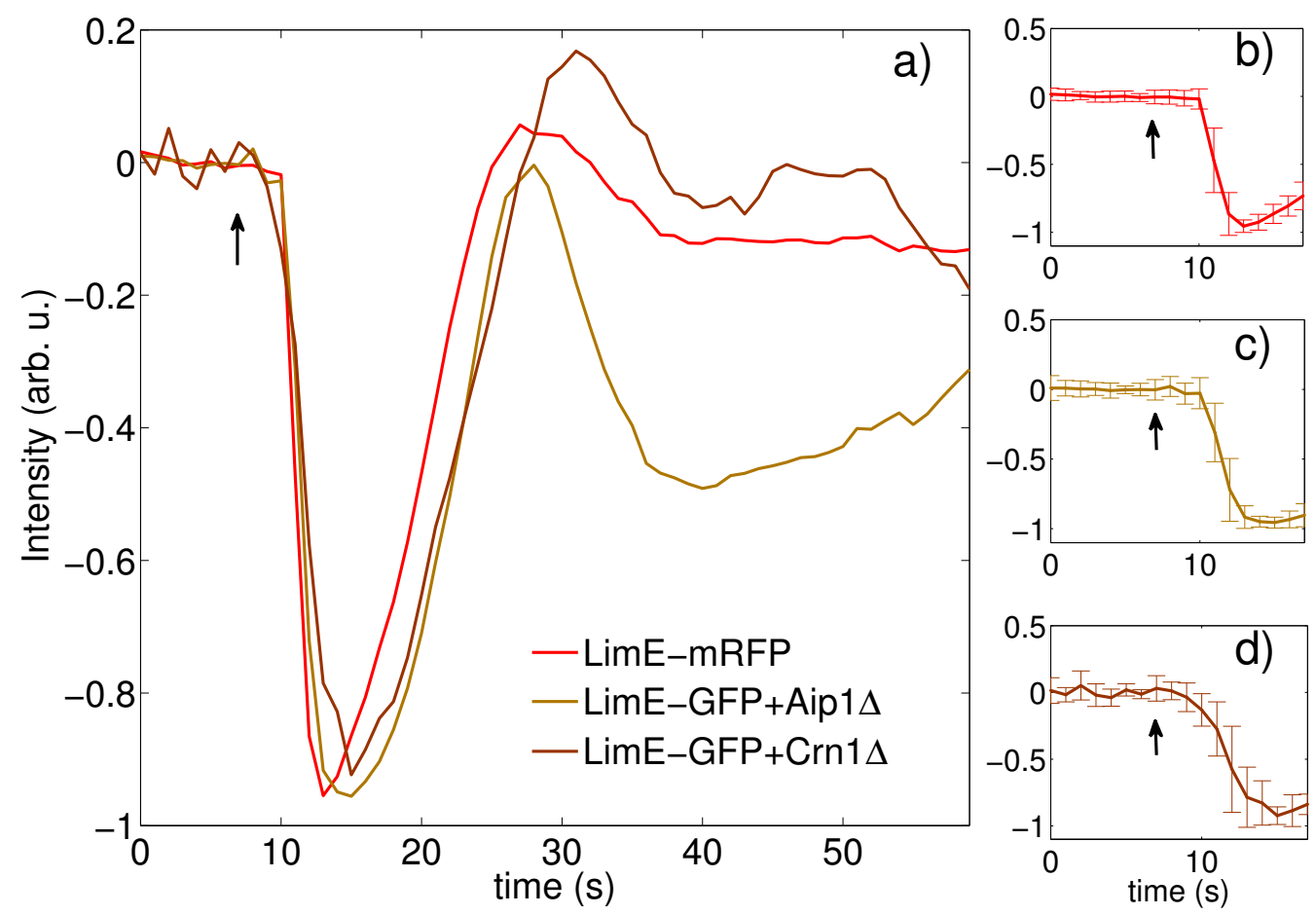

Figure 5.10: Time evolution of the mean cytosolic fluorescence. a) Averaged time series for LimE-WT (red), LimE-Aip1 $\Delta$ (gold) and LimE-Coronin 1A $\Delta$ (brown). b), c) and d) show the first $15 s$ of the time series with their corresponding errorbars for LimE-WT, LimE-Aip1 $\Delta$ and LimE-Coronin $1 \mathrm{~A} \Delta$ respectively. These were used to quantify the time for initiation of the translocation. 


\subsection{KNOCKOUT CELLS}

the wild type cells, after the overshoot the signal relaxes back to its basal levels, meaning the degree of polymerized filaments is recovered. Though in this case the signal has a second and strong drop, showing that the filaments are repolymerized to higher levels than their basal length. After this repolymerization, the signal relaxes very slowly to its steady state. It is evident that the lack of Aip1 in these cells resulted in a very weak depolymerization, consistent with the reports in literature where lacking Aip1 results in weak depolymerization [78, 70, 77].

The remaining LimE signals for Aip1 $\Delta$ also show different phenotypes, such as strong ringing (panel c), that differs from a sinusoidal waveform, as also broad overshoots (panel d). For the Coronin 1A $\Delta$ cells the waveforms are very similar but differ on the strength of their overshoot. For example in panel a) the overshoot is substantially higher, around 5 times higher than in the wild type. This is an indication that in these cells the depolymerization is strongly unregulated, as high fluorescence is an indicator of a high amount of depolymerized actin. The rest of the panels also show a higher overshoots than the wild type, but to different degrees.

\subsubsection{Concavity}

A biochemical network has a large amount of components, in Dictyostelium discoideum there are at least 138 proteins dedicated to the actin cytoskeleton [30]. This fact makes it hard to have specific information on the roles of Aip1 and Coronin $1 \mathrm{~A}$, and furthermore to predict the effects of their absence. In the previous section we have determined the possible roles of Aip1 and Coronin 1A by mapping the observed signals into symbols, suggesting a set of effective interactions between them. Inspired in this technique we established an interpretation of the concavity at the signal extrema.

We have seen that removing Aip1 or Coronin 1A changes dramatically the LimE response, and so the feedback felt by the actin cytoskeleton changes when removing one of these regulators. We can quantify how much the feedback has changed by measuring the concavity at the extrema of the time series. This interpretation can be developed by writing a generalized model for the observed protein 


\subsection{KNOCKOUT CELLS}
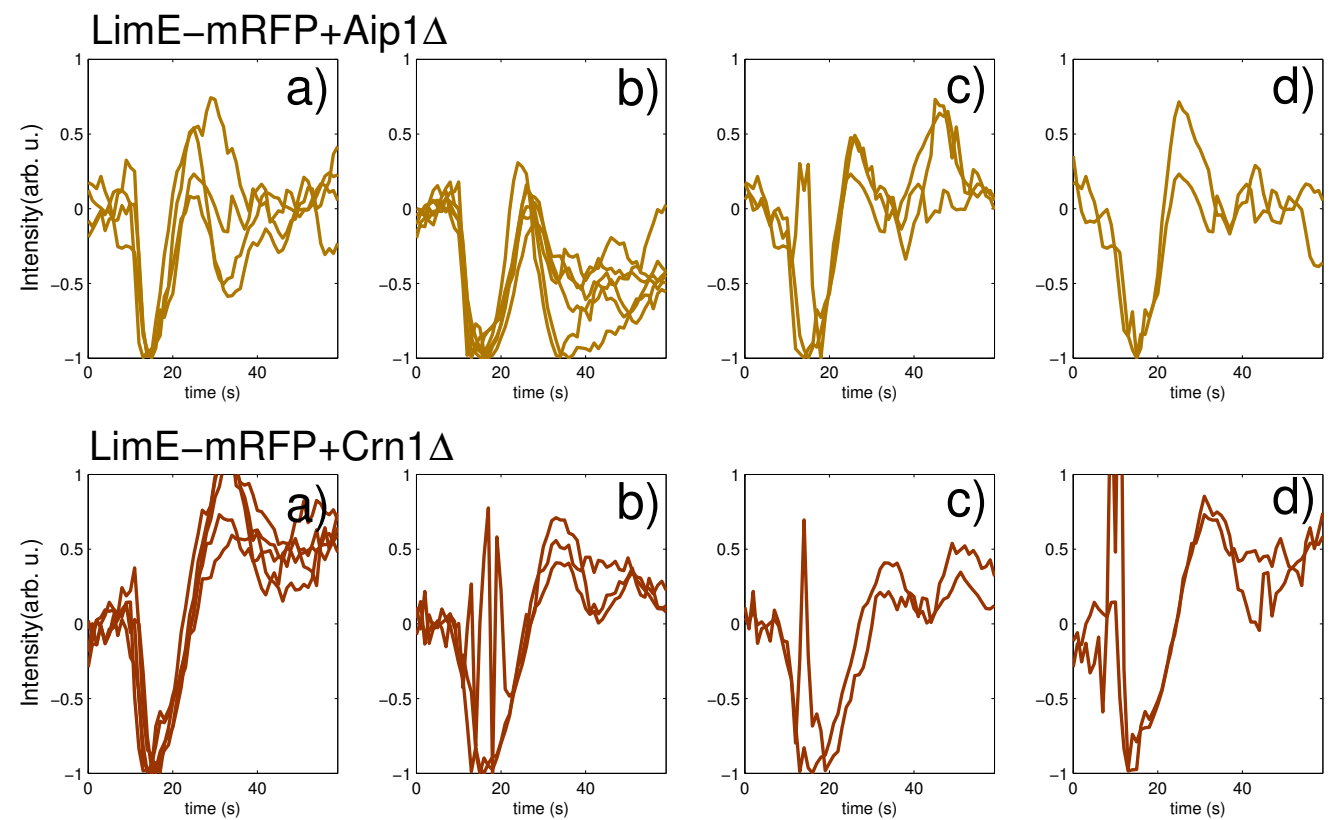

Figure 5.11: Different types of responses obtained in cells with genetical knockouts by applying the algorithm mentioned in section 5.2. The differences in the responses is higher than in the WT cells.. 


\subsection{KNOCKOUT CELLS}

$$
\frac{d x}{d t}=\sum_{n=0}^{\infty} a_{n} x^{n}+\rho(t)
$$

where the first term in the r.h.s. represents the linear and nonlinear self terms, which represent dissipation and autocatalysis, and the term $\rho(t)$ represent the feedback given by all the components interacting with $x$.

If we take the derivative of Equation (5.10) we get

$$
\frac{d^{2} x}{d t^{2}}=\sum_{n=0}^{\infty} a_{n} n x^{n-1} \frac{d x}{d t}+\frac{d \rho(t)}{d t}
$$

and similarly to the method of symbolic dynamics, we will concentrate in the extrema of the signals. If the extrema of the signal occurs at time $T$, by basic calculus rules we have

$$
\frac{d x(T)}{d t}=0
$$

which means that the feedback and the self terms balance each other. By plugging the values of the derivative of $x$ into Equation (5.11), we arrive to the following expression

$$
\frac{d^{2} x(T)}{d t^{2}}=\frac{d \rho(T)}{d t}
$$

which can be interpreted as a measure of the local feedback strength sensed by the protein at those times.

Therefore by measuring the concavity at the extrema we will gain information on how the feedback has changed. To visualize why this is a measure of the feedback strength and not only of the rate of change, we can analyze a simple example. Suppose that $\phi(t)$ depends on a variable $y$ such that $\phi(y(t))$. Then if an extrema is at $\left\{x_{o}, y_{o}\right\}$, by linearization Equation (5.10) becomes 


\subsection{KNOCKOUT CELLS}

$$
\frac{d x(T)}{d t}=\left(\sum_{n=0}^{\infty} n a_{n} x_{o}^{n-1}\right) x(T)+\frac{\partial \phi\left(y_{o}\right)}{\partial y} y(T)
$$

around the extrema, where the term in front of $y(t)$ is the feedback strength. Then by calculating the second derivative we get

$$
\frac{d^{2} x(T)}{d t^{2}}=\frac{\partial \phi\left(y_{o}\right)}{\partial y} \frac{d y(T)}{d t}
$$

which shows that the concavity is proportional to the strength of the feedback. Having in mind the scheme of Figure 5.9, it predicts that the removal of Aip1 or Coronin 1A must reduce the strength of the negative feedback, and consequently the concavity must be also reduced. We proceed to see how the concavity changes in the mutant cell lines compared to the wild type.

\subsubsection{Concavity distribution}

As the concavity at the extrema will give us a measure of feedback strength, we need to localize the maxima and minima for the knockouts time series. For that, we fitted a fourth order polynomial at $11 s \leq t \leq 19 \mathrm{~s}$ and at $10 s \leq t \leq 25 \mathrm{~s}$ for $\mathrm{LimE}-\mathrm{GFP}+\mathrm{Aip} 1 \Delta$ and LimE-GFP+Coronin $1 \mathrm{~A} \Delta$ respectively. We obtained the time to reach the minima for Aip $1 \Delta, \tau_{\operatorname{minAip} 1 \Delta}=7.90 \pm 1.12 \mathrm{~s}$ and for Coronin $1 \mathrm{~A} \Delta, \tau_{\text {minCoronin } 1 A \Delta}=7.59 \pm 1.33 \mathrm{~s}$. The mutation affects clearly the time to reach the minima as it takes longer to reach it. For Aip1 $\Delta$ cells some of the minima times overlap with those of the wild type. To further understand the effects of the mutation, we continued by analyzing the concavity distribution.

In Figure 5.12 we have the concavity distribution for the first minima in LimE for wild type (WT) and for the mutants Aip1 $\Delta$ and Coronin 1A $\Delta$ cell lines. In the mutants we can see that their concavity distribution is peaked in a value lower than for the wild type. The removal of Aip1 results in shifting the distribution peak to a value $($ Concavity $=0.027)$ below than a quarter of the WT value $($ Concavity $=$ 0.112). This means that the feedback has slowed down significantly taking a longer 


\subsection{KNOCKOUT CELLS}

time to turnover the polymerized network into depolymerization. Figure 5.14 a, shows the overlap of the time series around the minima, corresponding to the bin of Figure 5.12 (Aip1 $\Delta$ ) which contains the most cells, compared with the time series of the wild type. It is evident that the set of curves for Aip1 $\Delta$ (gold curves) have a much lower curvature compared to the wild type (red curves). Some of the Aip1 $\Delta$ time series show that the polymerized network is very stable for around $10 \mathrm{~s}$ , which is very slow compared to the turnover in the wild type. We can conclude that for the first minima, the absence of Aip1 results in a slower depolymerization since the feedback has slowed down substantially. This in agreement with the scheme of Figure 5.9 which predicted a slower turnover.

The concavity distribution peak in the Coronin $1 \mathrm{~A} \Delta$ cells also fall substantially to $($ Concavity $=0.069)$, Figure 5.14 b shows an overlap of the time series for Coronin $1 \mathrm{~A} \Delta$, corresponding to the bins with the highest amount of cells, also compared to the wild type. The effect is not as dramatic as in the absence of Aip1, but also we can conclude that is necessary for fast depolymerization at the minimum. Although in the scheme of Figure 5.9, a direct link is not found with the methods of [81, 82], it is inferred that Coronin 1A enhances the depolymerization by a cooperative effect with Aip1.

The change in the second extrema of the LimE signals will give insight into the transition from depolymerization to polymerization. The concavity distributions for this case are shown in Figure 5.13, it is remarkable that for the wild type the distribution is very peaked showing that this part of the signal is very robust between cells. Also the concavities have a value of -.0086 which is $7 \%$ of the absolute value for the minima. Therefore such a small value of concavity is due to the stationary feedback that is received in the WT. The concavity distribution for the knockouts results in absolute values higher than for the wild type, meaning that the feedback is enhanced at this section of the time series. In this case the Aip1 $\Delta$ distribution is peaked around the value Concavity $=-0.0554$, which means that the magnitude of this value is $72 \%$ higher than in the wild type. So opposite than in the minima here the absolute concavity became higher. This implies that the transition from effective depolymerization to polymerization is enhanced, this asymmetry is not contemplated in the scheme of Figure 5.9 .

The absence of Coronin 1A also results in concavity values with higher mag- 


\subsection{INTERPRETATION}

nitude at the maxima. The distribution has two peaks at Concavity $=-0.0186$ and Concavity $=-0.0377$, therefore it has a similar effect as the Aip1 knockout but with lower magnitude. Therefore we can conclude that the effects of Aip1 and Coronin $1 \mathrm{~A}$ are asymmetric with respect to the polymerization to depolymerization transition and the depolymerization to polymerization transition. Aip1 and Coronin $1 \mathrm{~A}$ increases the depolymerization transition in the wild type, this is in agreement with the literature reviewed in the Background chapter. Where effects like filament bursting [59, 14] and increased sensitivity of ADP-rich filaments [36] were discussed. The second effect of enhanced polymerization can be due to indirect regulation of polymerization nucleation factors. It is known that Coronin $1 \mathrm{~A}$ acts as an inhibitor of the Arp2/3 complex at high concentrations [18]. Therefore it is possible that since Coronin $1 \mathrm{~A}$ is not present, the Arp2/3 complex is not as strongly regulated and polymerization is promoted. Such an effect has not been recorded for Aip1 so far, therefore the enhancement of depolymerization and the inhibition of depolymerization could be a cooperative effect between these two proteins.

\subsection{Interpretation}

In this section we give a heuristic interpretation of our observations. We have observed the translocation of LimE, Coronin 1A and Aip1 from cytosol to the cortex induced by external cAMP stimulation. The average cytosolic fluorescence of LimE, presents a characteristic response after stimulation. There is an initial drop to lower levels of fluorescence, then an overshoot followed by the recovery to its steady state, this response is reminiscent to a damped oscillation. Even though there is substantial cell to cell variability in our observed cells, there are similarities between the responses. For example, after stimulation the average cytosolic signal for LimE is the first one to reach its minimum, then its followed by either Coronin $1 \mathrm{~A}$ or Aip1. We have quantified the sequential order in which the minima are reached and found that the first one is LimE, followed by Coronin 1A, and then by Aip1, the maxima is also found in the same sequential order.

Using a proposed algorithm to convert the observed signals into symbolic dynamics [81, 82], we have extracted a possible feedback loop in which LimE activates 


\subsection{INTERPRETATION}
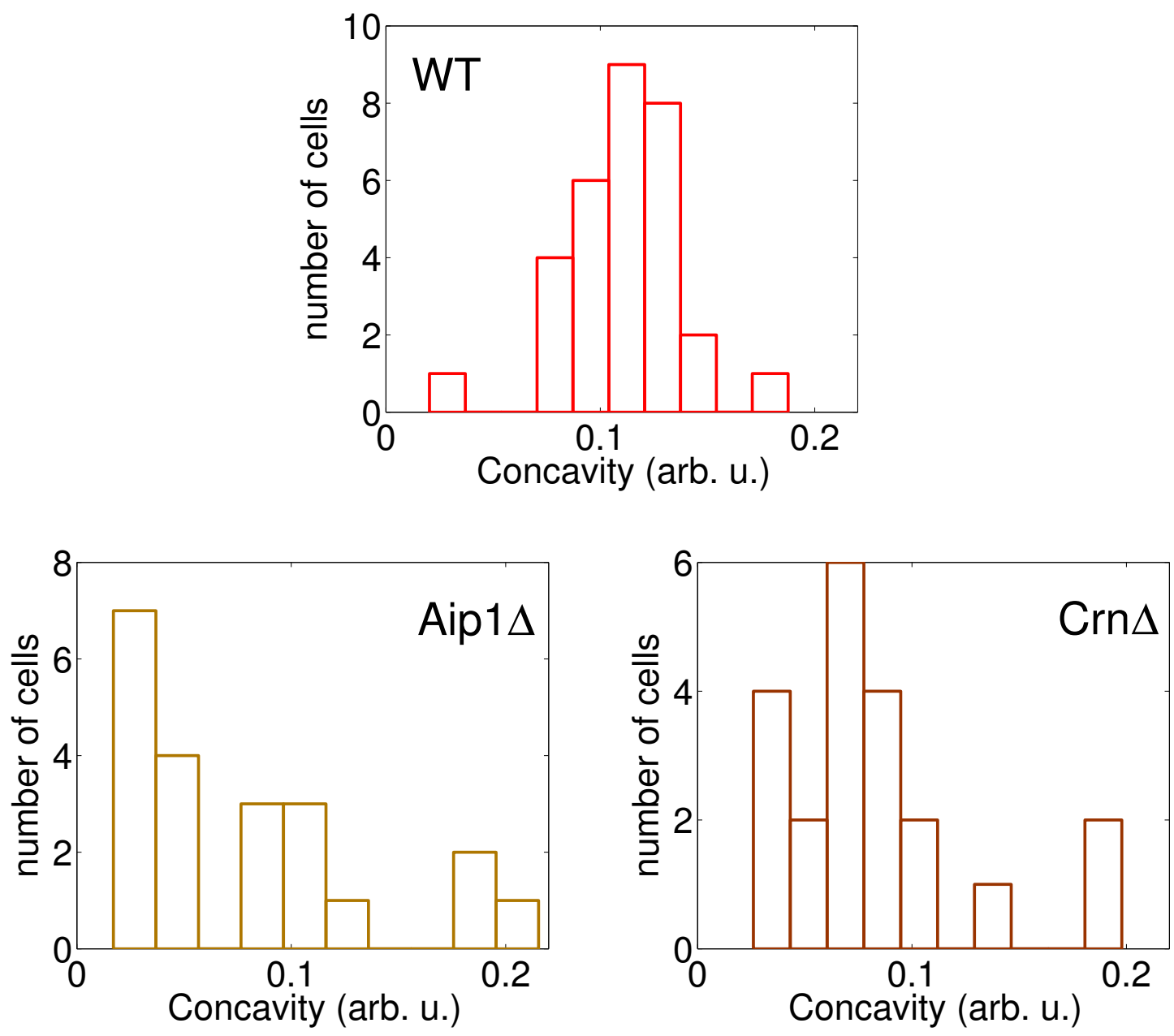

Figure 5.12: Concavity distribution measured at the first minima after stimulation of the LimE responses for wild type (red), Aip1 $\Delta$ (gold) and Coronin $1 \mathrm{~A} \Delta$ (brown) cells. 


\subsection{INTERPRETATION}
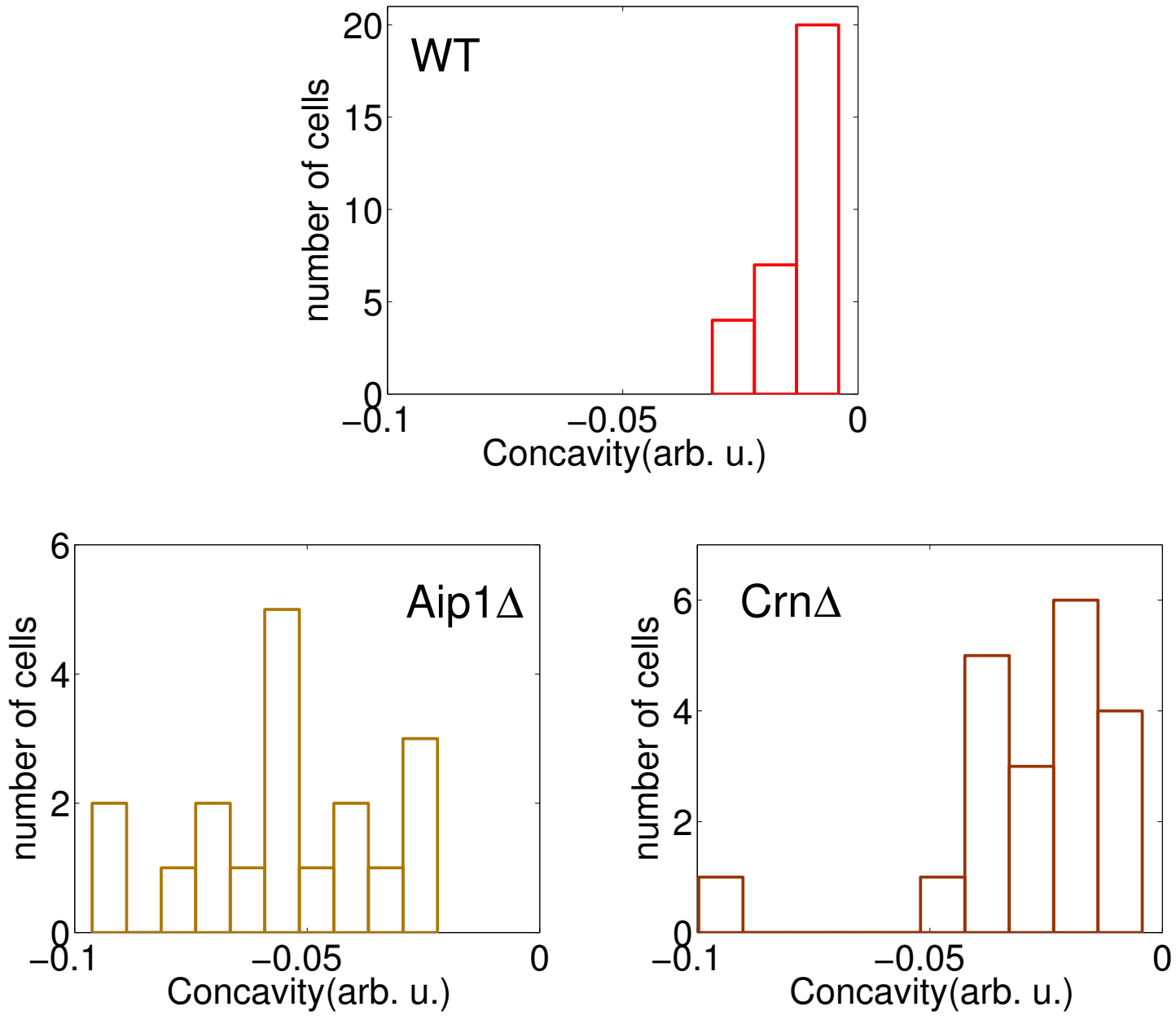

Figure 5.13: Concavity distribution measured at the first maxima after stimulation of the LimE responses for wild type (red), Aip1 $\Delta$ (gold) and Coronin $1 \mathrm{~A} \Delta$ (brown) cells. 


\subsection{INTERPRETATION}
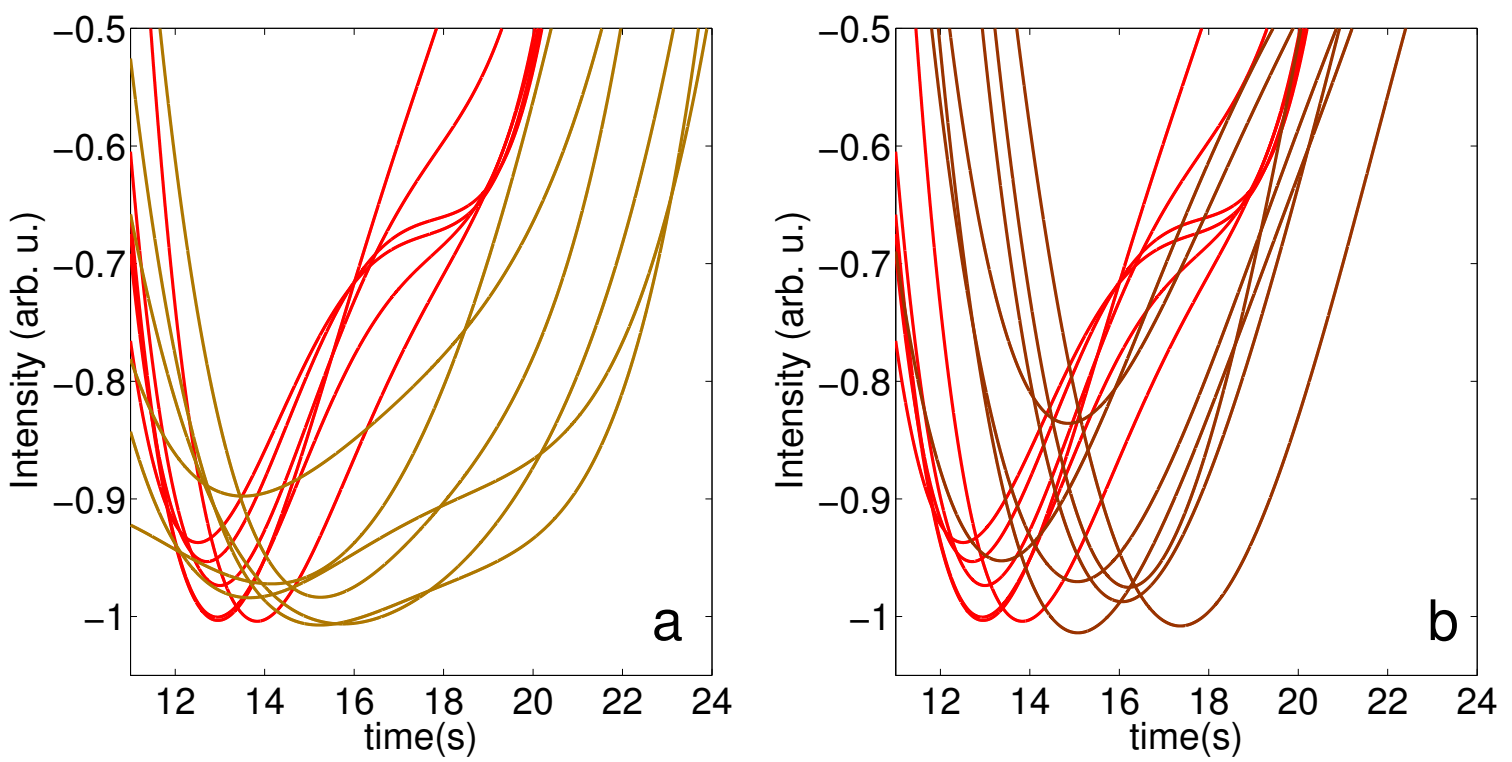

Figure 5.14: Comparison of the concavities at the first minima between: a, wild type (red) and Aip1 $\Delta$ (gold) cells, and b,wild type (red) and $\operatorname{Crn} \Delta$ (brown) cells.

Coronin 1A, Coronin 1A activates Aip1, and Aip1 inhibits LimE. This loop predicted that in the absence of either Aip1 or Coronin 1A the inhibition of LimE will be compromised. Figure 5.15 shows our interpretation of this scenario. In the first panel we have a polymerized structure (actin monomers denoted by red circles) at the cell cortex, this structure is the one formed by external cAMP stimulation and corresponds to the observed minimum in the LimE time series. Suppose the existence of an inhibitory molecule that represent all the molecules that inhibit the cytoskeleton (denoted by gray circles in panel 2), the interaction of this molecule becomes weaker in the absence of Aip1 $\Delta$ and Coronin $1 \mathrm{~A} \Delta$ cell lines.

As the negative feedback has been compromised in the knockout cell lines, there is a slow build up of the inhibitory molecule and takes longer to inhibit the polymerized structure (panel 2, Figure 5.15). As the build up is slower the depolymerization also becomes slower, and as the cytoskeleton is depolymerizing the monomers return to the cytosol. As a consequence of the positive feedback given to the inhibitory molecule it will retract back to the cytosol slowly. During this retraction is where the maxima of LimE is observed in our time series. Therefore the translocation of both species from cytosol to cortex and back and forth is 


\subsection{INTERPRETATION}

slowed down for both of them. A simple physical analogy would be the dynamics of a damped oscillator, in such a system the reduction of the negative feedback results in lower frequencies. We conjecture without proof that this should hold even in the presence of nonlinear interactions, as long as the interactions remain monotone, meaning that the inhibitor is always an inhibitor and the activator is always an activator.

We have observed in the previous section lower concavity at the minima for the time series of the knock outs cell line. Therefore the first two panels of Figure 5.15 are in agreement with experiment, but the observed maxima became narrower in the knock outs. Figure 5.16 shows a modified scheme from the previous one that resembles our experimental observations, it differs in the last two panels. It is useful to use as a guide the signal shown in Figure 5.11 b for Aip1 $\Delta$, this extreme case simplifies the interpretation. In panel 3, the scheme starts to differ from the earlier one, as the levels of the inhibitory protein are high in the cytosol there is a sudden decrease on their influence on the cytoskeleton. This can be interpreted as if the inhibitory proteins suddenly leave the cytosol, leaving room to the actin monomers to polymerize, or a sudden desensitization of actin from the inhibitory proteins. Then the former actin structure is created again as there is no inhibition and is free to polymerize.

We conjecture that such interaction must be mediated by another actin binding protein that we are not observing. With this scenario in mind, the absence of Coronin $1 \mathrm{~A}$ or Aip1 results in a weaker depolymerization process but also in a very strong polymerization. It has been discussed in Chapter 2 that these proteins confine capping proteins [70] and cofilin [36] indirectly to certain positions of the cytoskeleton, this could not be the explanation since the role of those proteins is to limit the growth and sever actin filaments respectively. Coronin $1 \mathrm{~A}$ has been shown in vitro to have inhibitory interactions with the Arp2/3 complex [18] which enhances polymerization. It is possible that in vivo Coronin 1A and Aip1 inhibit cooperatively the Arp2/3 complex. It would be interesting to label other proteins in these mutants to see if their activity is enhanced in the knockouts. With this heuristic interpretation we hypothesize that there must be a set of proteins that inhibit suddenly the action of inhibitory proteins, or that promote polymerization strongly. 


\subsection{INTERPRETATION}
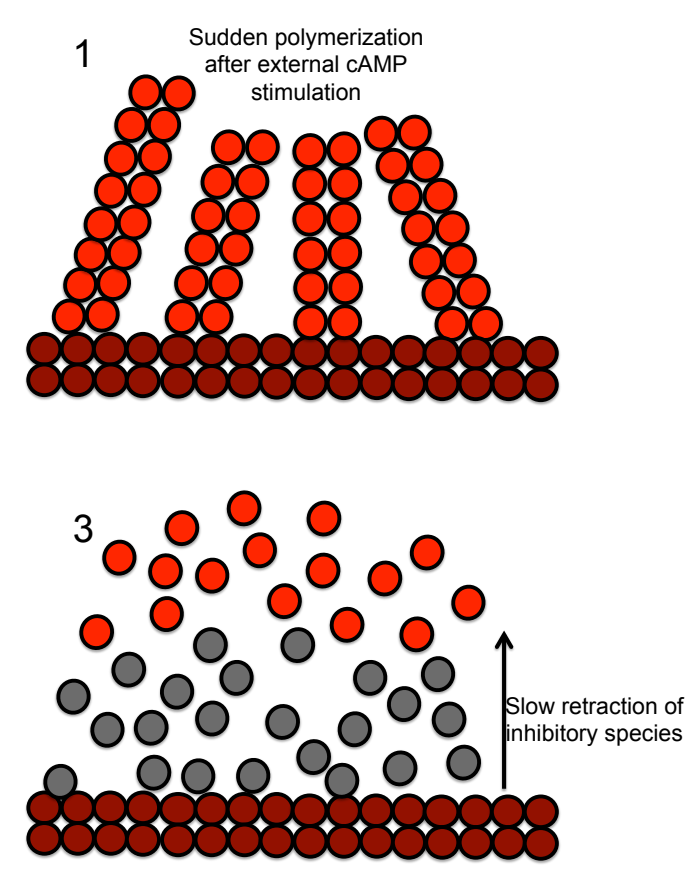
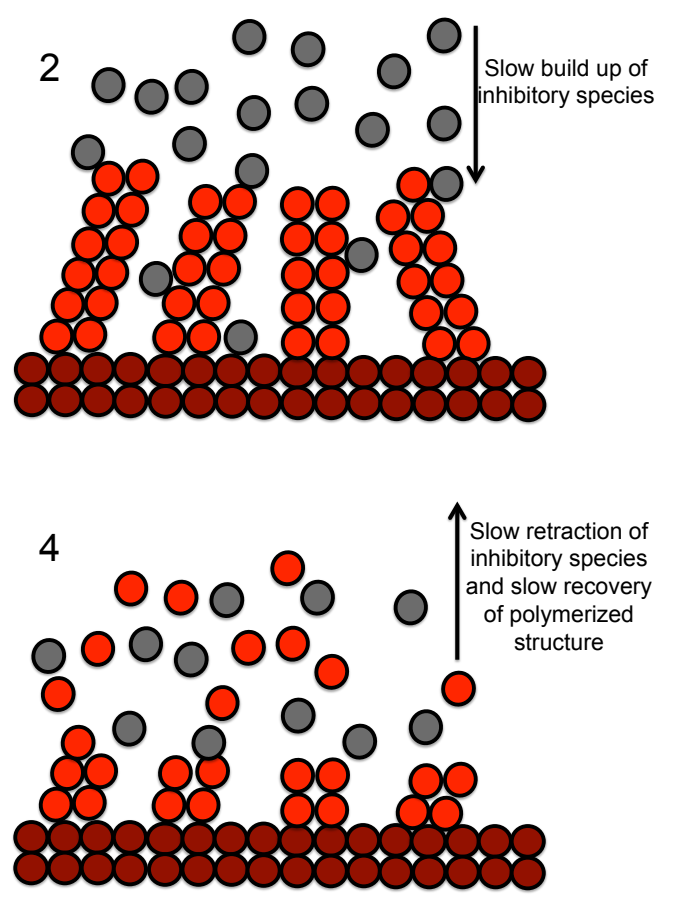

Figure 5.15: Interpretation of the scheme predicted by the symbolic dynamics in the absence of Coronin 1A or Aip1. In panel 1, we have the sudden polymerization induced by cAMP stimulation. In 2 is a very slow build up of an inhibitory protein that induces the disassembly of the cytoskeleton. Then there is a slow retraction of the inhibitory protein to the cytosol in panel 3, and finally in panel 4 a very slow recovery of the polymerized structure. 


\subsection{INTERPRETATION}
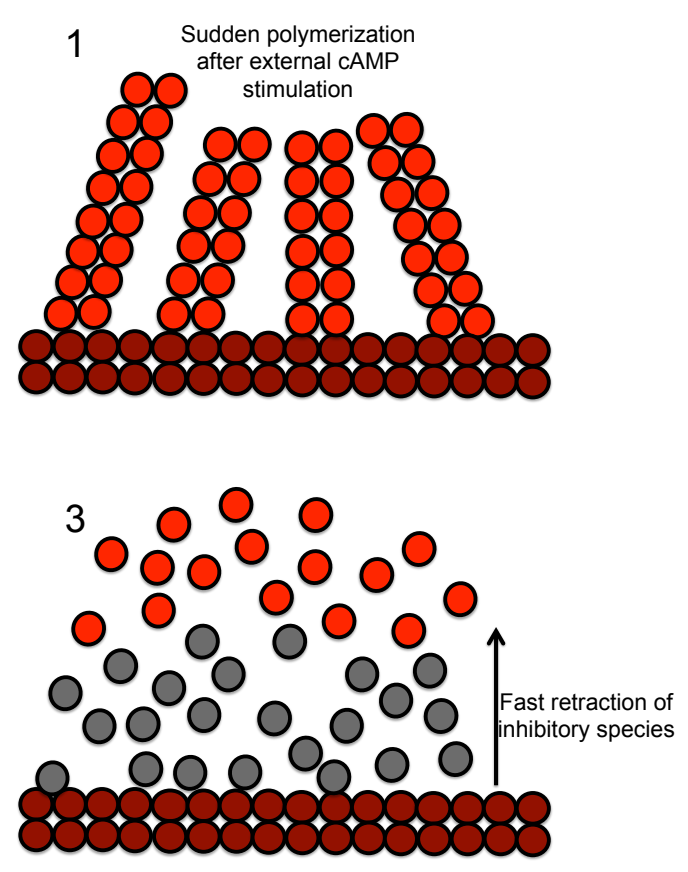
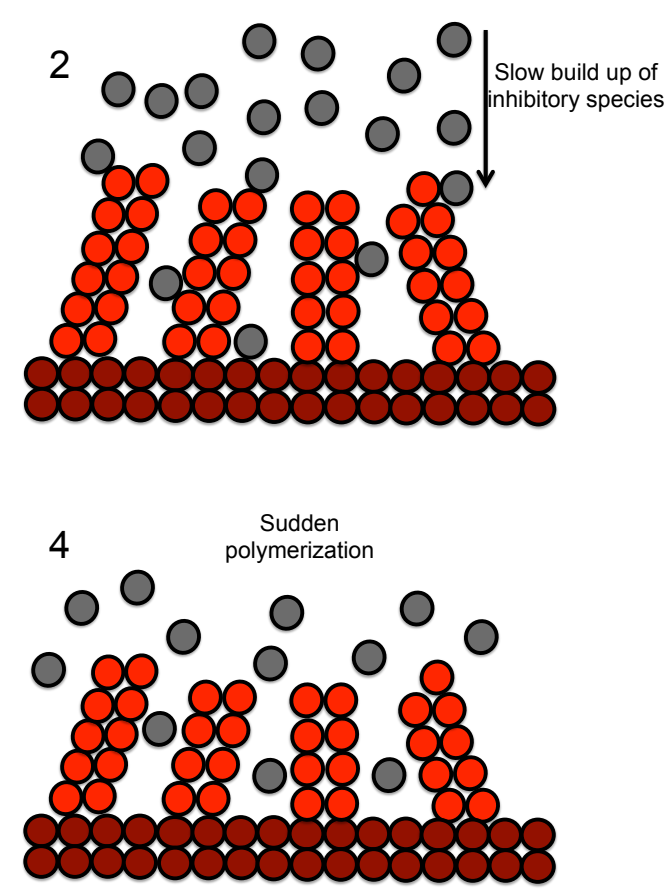

Figure 5.16: Interpretation of the experimental results, panels 1 and 2 are the same as in Figure 5.15. In panels 4 and 3 shows a sudden retraction or desensitization of the inhibitory protein resulting in a sudden polymerized structure. 


\subsection{CHAPTER SUMMARY}

\subsection{Chapter summary}

In this chapter we have studied the transient polymerization/depolymerization observed in Dictyostelium discoideum when stimulated with a single pulse of cAMP. Coronin 1A and Aip1 were known to act cooperatively to enhance the disassembly of filaments [59, 14], this knowledge was acquired by a series of in vitro and in vivo experiments. It is known that Coronin 1A inhibits polymerization by interacting with the Arp2/3 complex [18], while an indirect regulation of capping proteins was known for Aip1 [70].

The time series for the LimE average cytosolic intensity showed a decrease followed from the stimulation, then an overshoot and finally a relaxation to a steady state. In the first decrease the actin cortex becomes brighter implying the growth of actin filaments and during the overshoot it became darker implying a decrease in filament size. Therefore at the times that LimE has a negative slope is interpreted as polymerization while positive slopes as depolymerization.

It was found that the LimE, Aip1 and Coronin 1A translocated in a sequential manner, the progression was the following: Aip $1 \rightarrow$ LimE $\rightarrow$ Coronin $1 \mathrm{~A}$. To infer the possible causality and interactions between these proteins we have used the algorithm proposed in [81, 82, which consists in transforming the observed time series into symbolic dynamics. The effective feedback loop extracted was LimE enhances Coronin 1A, Coronin $1 A$ enhances Aip1 and Aip1 inhibits LimE. The prediction of this loop was that in the absence of Aip1 or Coronin 1A the turnovers at the minima and the overshoots would be slowed down.

To prove the degree of agreement and deviation with this scheme, LimE was measured in cell lines where either Coronin 1A or Aip1 were genetically removed. The time series of these cell lines highly differ from that of the wild type. To quantify the degree of deviation, we have measured the concavity in the extrema of those signals. It was proved that the concavity is a direct measured of the rate of change, and an indirect measure of the strength of the feedback acting on LimE. Therefore by measuring concavity we obtain useful information on the regulatory feedback, without any detailed knowledge of the involved dynamics for LimE.

We have observed a large cell to cell variability in the response waveform of LimE for the knockout cell lines, this was reflected also by their concavity distri- 


\subsection{CHAPTER SUMMARY}

bution. For both Aip1 and Coronin 1A the concavity decreases for the minima, the slowing down in the feedback implies a decrease in the capability to exert the inhibition to LimE. This is in agreement with the scheme obtained with symbolic dynamics. In the maxima the effect has been the opposite, the absolute values of the concavity increased, resulting in sharper overshoots which imply very strong polymerization. This is not in agreement with the scheme obtained, therefore there must be an asymmetry in the interactions with LimE. The removal of this proteins results in inhibited depolymerization and enhanced polymerization.

In the last section we have introduced an interpretation of our data, where we proposed an indirect regulation of Coronin 1A and Aip1 for inhibiting polymerization. In the literature survey done in the Background chapter, it has been shown that high doses of Coronin 1A inhibits the binding of the Arp2/3 complex to actin filaments [18]. Therefore we conclude that in the absence of these two proteins not only results in decreased depolymerization but also in an increased polymerization. 
5.6. CHAPTER SUMMARY 


\section{Chapter 6}

\section{Conclusions and discussion}

This work has been dedicated to study the dynamical properties of the actin cytoskeleton, as well the regulation exerted by Coronin 1A and Aip1. We have used the chemotactic amoeba Dictyostelium discoideum during the starvation stage as a model organism to study these matters. By means of flow photolysis we were able to perturb the basal dynamics of the cells by stimulating them by a short pulse of cAMP. During this stimulation the cell presents a physiological response where a transient actin structure is created. The dynamics of the actin cytoskeleton can be tracked by the indirect marker LimE-mRFP and the average cytosolic fluorescence is a measure of its translocations. When the cell is stimulated with a pulse of cAMP the fluorescence levels deplete, then they overshoot and finally relaxes back to equilibrium, the observed time trace is reminiscent to the response of a damped oscillator.

In a previous study we have observed that the cell cytoskeleton exhibit oscillatory properties such as a resonance frequency and the exhibition of self sustained oscillations [114]. In this work we proceeded in investigating the dynamical properties of cells exhibiting self sustained oscillations and co-expressing LimEmRFP+Coronin 1A-GFP or LimE-mRFP+Aip1-GFP. To identify them and characterize their properties, we did an statistical analysis on the measured time series via the autocorrelation function. We have found many interesting properties such as: an integral relation between the fluorescence values of LimE and pseudopod extension, indications of an inverse relation between the times series variance and 
correlation time, and the exhibition of different transients after stimulation. We have proposed a couple of well supported hypothesis on the mechanisms behind our observations, giving an intuitive perception of the biophysical processes

The role of fluctuations in chemical and biological systems has been discussed since the early works of far from equilibrium thermodynamics [87, 88]. We have shown that the actin cytoskeleton is no exception, and we have proposed two hypotheses regarding the type of fluctuations that explain the observed physical properties. In the first scenario the system is very close to criticality and susceptible to environmental noise, the analysis has shown that the majority of cells are strongly dominated by stochastic effects. For self oscillating cells the majority shows a relative noise contribution (RNC) of at least 35\%, its is conjectured that cells take advantage of this for having a high sensitivity to internal changes and maintaining high amplitudes at low metabolic costs. In the second hypothesis the competition between internal and external fluctuations explain the observed relations between the signal variance and its correlation time. For this we used a phase reduction method to reproduce the properties of our observed autocorrelation functions. The main component of the second model is a multiplicative noise term coupled with the feedback of the oscillator, this component decorrelates the observed time series at high amplitudes. The physical interpretation is the corruption of the feedbacks between the cytoskeleton and its regulators, possibly done by molecules in low copy numbers that are in charge of mediating the interaction between these proteins.

Even though our theoretical hypothesis matches well our observations, we need to be particularly careful in finding the set of parameters where are valid. The phase reduction approximation date backs to 1967 from the work of Melvin Lax [60], but recently a debate of its validity has been reopened [122, 103], in particular on the usage of the Stratonovich and Ito interpretations for Stochastic calculus. Both interpretations infer different physical scenarios, Ito calculus is suitable when assuming that the forcing fluctuations consists of discrete pulses while Stratonovich calculus assumes that it is a continuous function [107]. This debate is not open only in the phase approximation of dynamical systems, but also in the experimental observation of general physical systems such as electrical circuits [80]. We have addressed these issues conducting further investigations of 
the Fokker-Planck formalism for self sustained oscillators. It has been concluded that correlation time $\left(\tau_{c}\right)$ is poorly estimated from short time series in comparison with theoretical estimates. On the other hand the variance $\left(\sigma^{2}\right)$ is well estimated and suitable for comparison with theory making the analysis of the first hypothesis reliable, while the second one remains a possibility but it is indistinguishable by our current methods. We have studied only the statistical properties of the time series neglecting its local properties, we can learn the lessons from spiral defect chaos, where it has been shown that both the statistical [72] and local [29] properties play an important role. Further studies must give insightful results since a motile cell is changing parameters over time.

In the background chapter we have reviewed the different times scales in which different components of the actin cytoskeleton and the signaling system operate. We have shown an interplay between fast and slow time scales in the response dynamics of some cells classified as oscillatory. A small percentage of cells with high variance, and in some cases high correlation time, in their time series, stopped their activity after stimulation (shown in Figure C.1). At a time of around $100 \mathrm{~s}$ after stimulation the oscillatory activity is recovered. A theoretical analysis of these time series has shown that these transients cannot be explained by the nonlinear interactions between a single activator and a single inhibitor. It was shown that is necessary to add another variable that is quiescent during oscillatory activity, where the driving to higher amplitudes activates this variable and terminates the oscillations. This variable would represent a physical process that is maintained active for $\sim 10$ oscillation periods, the components of the signaling system, such as PIP3 and PTEN, are ideal candidates to be investigated during this process since they present dynamics with time scales of the same order.

Several tools from the areas of applied mathematics will help to interpret better future data, for example in control theory a set of tools have been developed to infer the dynamics of a system with the minimum amount of observations [52]. A recent remarkable observation has shown that is possible to determine the minimum number of observations via a graphical method [64, 65] which avoids the need to do extremely tedious calculations. Unfortunately to do the full reconstruction of the dynamics their interactions must be known, it was particular frustrating for the author not to find generalized theorems for the observations of limit cycles. 
The following question arose during this research: If one particular element of a physical system is showing periodic dynamics, what can we infer for the non observed elements?. This question originated since we have observed periodic oscillations in LimE, Coronin 1A and Aip1, in which we have shown the existence of causal interactions between them. If such a question is answered soon, it would be utterly useful for interpreting future experiments where more elements of the actin cytoskeleton and signaling system are observed during these periodic oscillations.

In the second part of this work we endeavoured in studying the regulating properties of the cytoskeleton, for that we studied the dynamical properties of the damped fluorescence oscillations exhibited by LimE, Coronin 1A and Aip1. The latter and former proteins has been known to have cooperative effects to disassemble the barbed end of the actin cytoskeleton as well to protect the pointed end. We have dealt with the natural cell to cell variability, in this work we addressed this issue by developing a simple algorithm for sorting data and study the biophysical features of single cells. The possible artifacts due to averaging have been discussed, this is not the first time that the problem is addressed. In studies of the p53 system, that assists in DNA repair, due to averaging the researchers thought for a long time that the system exhibited damped oscillations when stimulated, but in reality the system exhibited self sustained oscillations [7.

Among the biophysical properties of cAMP response time series we have observed a sequential translocation of these proteins, were Aip1 translocated first, followed by LimE and the by Coronin 1A. The times to reach the extrema of the signals were also sequential were LimE reached first the minimum, followed by Coronin 1A and then by Aip1, for the maximum the order was the same. This information was useful to translate the observed dynamics into symbolic dynamics [81, 82, from this we can extract an inferred effective feedback loop. We concluded that the symbolic dynamics were in accord with a loop where LimE activated Coronin 1A, which activated Aip1, which inhibited LimE. We interpreted as a prediction from this graph that the negative feedback loop will be weakened in the absence of Coronin 1A or Aip1.

To confirm the proposed hypothesis we proceeded by studying the LimE response in cells lacking either Aip1 or Coronin 1A. We have found remarkable properties in these responses such the maintenance of actin structures for long 
times, as well a higher variability on single cell response. We have chosen to measure the concavity at the extrema of the signal to characterize the changes between the response from wild type cells to those of the mutants. We have proven that the concavity at the extrema is a measure of the feedback felt by actin, this is particularly useful since we lack detailed knowledge on the interactions between the components. Remarkably we have found an asymmetry on the concavities at the extrema of the knockout cell lines. For the minima we have found that the curvature of the signals was always lower, showing that the feedback rate of change (and indirectly the strength) has decreased but at the maxima of the signals the curvature was higher showing an increased feedback. Showing that the scheme obtained from the symbolic dynamics is in agreement at the minima of the signal but not on the maxima.

A hypothesis has been discussed for interpreting the data that we obtained. We start by supposing the existence of an inhibitory specie that disassembles the actin cytoskeleton, this specie will translocate to the cell cortex whenever actin is polymerized, since actin exerts a positive feedback on the inhibitory specie. If the negative feedback exerted by this specie is weakened, it will result also in a weak positive feedback. Therefore it takes longer to translocate to the cell cortex and the actin structures are maintained for longer times. Once it has inhibited the actin at the cell cortex, it will translocate back to the cytosol slowly and therefore the recovery would be slower. This would have been reflected in the experimental data as a lower concavity at the maxima. Therefore we hypothesized a sudden desensitization of the cytoskeleton to the influence of these species happens, or a sudden release retreat back to the cytosol of the inhibitory species. Therefore an unobserved component must be in charge of inhibiting the activity of the inhibitor, and in WT cells this unobserved specie is regulated. A possible candidate for this unobserved specie is the ARP2/3 complex, since it promotes nucleation of actin filaments and it has been shown to be inhibited Coronin 1A [18].

In this work we have shown that we are able to study the dynamics of the cell and learn about their internal biophysical processes in spite of our ignorance on the distribution of internal parameters, which are reflected in the variability of responses and different dynamical states. Still in the future it will be necessary to be able to manipulate the genetics of the Dictyostelium discoideum up to the 
point of controlling their expression levels. This will allow us to study explicitly the physical properties and bifurcation structures behind the mechanisms inside the cell. In E. coli this is possible, for example Leibler, Barkai and Alon have studied the adaptation properties to aspartate by manipulating the expression levels of CheR [2]. But also the physicist need to become familiar with the biological tools available. It is the hope of the author that this study will help to the further understanding of the connection of the actin cytoskeleton with the signaling system, as well the regulation of the cytoskeleton in-vivo. 


\section{Appendix A}

\section{Analytical and numerical calculations of correlation time $\tau_{c}$ and variance $\sigma^{2}$}

\section{A.1 Correlation time in multiplicative noise model}

In section 4.5.2 we have proposed the following model for the self sustained oscillations observed in the actin cytoskeleton

$$
\frac{d z}{d t}=\left(\lambda+i \omega_{o}-\epsilon|z|^{2}\right) z-i \sqrt{D_{1}} \xi_{1}(t)|z| z+\sqrt{D_{2}} \xi_{2}(t)
$$

since the system is defined in the complex plane then we can transform Equation (A.1) into polar coordinates by rewriting in term of amplitude and phase as $z(t)=$ $a(t) \exp (i \phi(t))$, then we get

$$
\begin{array}{r}
\frac{d a}{d t}=\lambda a-\epsilon a^{3}+\sqrt{D_{2}} \xi_{2}(t) \cos (\phi) \\
\frac{d \phi}{d t}=\omega_{o}-\sqrt{D_{1}} a \xi_{1}(t)-\frac{\sqrt{D_{2}}}{a} \xi_{2}(t) \sin (\phi)
\end{array}
$$

we can also rewrite the definition for the autocorrelation function in polar coordinates, and by defining a variable transformation as $\psi=\phi-\omega_{o} t$ 


\section{A.1. CORRELATION TIME IN MULTIPLICATIVE NOISE MODEL}

$$
\begin{array}{r}
\left\langle z(t-\tau) z^{*}(t)\right\rangle=a^{2} \exp \left(-i \omega_{o} \tau\right)\langle\exp (i(\psi(t-\tau)-\psi(t)))\rangle \\
=\sigma^{2} \exp \left(-i \omega_{o} \tau\right) \exp \left(-\frac{\left\langle(\psi(t-\tau)-\psi(t))^{2}\right\rangle}{2}\right)
\end{array}
$$

where we have approximated the variance as $\sigma^{2}=a_{o}^{2}$, where $a_{o}$ is the is the steady state amplitude of the oscillator in the absence of fluctuations. The transform of the exponential term is given by the fact that $\psi$ is a Gaussian variable 60 .

Therefore the phase equation can be rewritten as

$$
\frac{d \psi}{d t}=-\sqrt{D_{1} \sigma^{2}} \xi_{1}(t)-\sqrt{\frac{D_{2}}{\sigma^{2}}} \xi_{2}(t) \sin \left(\psi+\omega_{o} t\right)
$$

and its time evolution will be given by

$$
\psi(t)-\psi(t-\tau) \approx-\int_{t-\tau}^{t} \sqrt{D_{1} \sigma^{2}} \xi_{1}(s)+\sqrt{\frac{D_{2}}{\sigma^{2}}} \xi_{2}(s) \sin \left(\omega_{o} s\right) \mathrm{d} s
$$

where we have used the approximation $\psi+\omega_{o} t \rightarrow \omega_{o} t$, commonly used in engineering [60]. We can calculate the mean of the square of A.5

$$
\begin{array}{r}
\left\langle(\psi(t-\tau)-\psi(t))^{2}\right\rangle=\left\langle(\psi(t)-\psi(t-\tau))^{2}\right\rangle= \\
D_{1} \sigma^{2} \int_{t-\tau}^{t} \int_{t-\tau}^{t}\left\langle\xi_{1}\left(s_{1}\right) \xi_{1}\left(s_{2}\right)\right\rangle \mathrm{d} s_{1} \mathrm{~d} s_{2} \\
+2 \sqrt{D_{1} D_{2}} \int_{t-\tau}^{t} \int_{t-\tau}^{t}\left\langle\xi_{1}\left(s_{1}\right) \xi_{2}\left(s_{2}\right)\right\rangle \sin \left(\omega_{o} s_{2}\right) \mathrm{d} s_{1} \mathrm{~d} s_{2} \\
+\frac{D_{2}}{\sigma^{2}} \int_{t-\tau}^{t} \int_{t-\tau}^{t}\left\langle\xi_{2}\left(s_{1}\right) \xi_{2}\left(s_{2}\right)\right\rangle \sin \left(\omega_{o} s_{1}\right) \sin \left(\omega_{o} s_{2}\right) \mathrm{d} s_{1} \mathrm{~d} s_{2}
\end{array}
$$

since the fluctuating variables are defined as $\left\langle\xi_{i}\left(s_{1}\right) \xi_{i}\left(s_{2}\right)\right\rangle=\delta\left(s_{1}-s_{2}\right)$ and $\left\langle\xi_{i}\left(s_{1}\right) \xi_{j}\left(s_{2}\right)\right\rangle=0$ we can calculate the integrals in A.6 as 


$$
\begin{array}{r}
\left\langle(\psi(t-\tau)-\psi(t))^{2}\right\rangle= \\
D_{1} \sigma^{2} \tau+\frac{D_{2}}{\sigma^{2}}\left(\frac{\tau}{2}-\frac{\left.\sin \left(2 \omega_{o}(t-\tau)\right)-\sin \left(2 \omega_{o}(t)\right)\right)}{4 \omega_{o}}\right) \approx \\
\frac{2 D_{1} \sigma^{4}+D_{2}}{2 \sigma^{2}} \tau
\end{array}
$$

finally the correlation time is given by

$$
\tau_{c}=\frac{4 \sigma^{2}}{2 D_{1} \sigma^{4}+D_{2}}
$$

In Figure A.1 we can see the comparison between analytical and numerical calculations. The phase equation A.2 was simulated by varying the parameter $\sigma$ and keeping $D_{1}$ and $D_{2}$ constant. For each value of $\sigma, 100$ realizations were simulated using the Euler-Maruyama algorithm. The cloud of black dots are single realizations of the obtained values of $\tau_{c}$ and the red curve represent their mean value. We can see that the average values of correlation time and the analytical prediction are in close agreement.

\section{A.2 Numerical validation for short time series}

We have checked the effects of determining parameters from short time series by doing numerical simulations. Equation 4.4 was simulated using the EulerMaruyama algorithm (details in Appendix B) for long time series of $10000 s$ and ensembles of short time series of $100 \mathrm{~s}$. Notice that for the correlation time there is a strong disagreement between the correlation time of long time series (black line) vs the mean of the ensemble for short time series (red dashed line), also the variance is very large (denoted by the colored area). It can be seen that the variance for long time series and the mean for short time series are in good agreement, also the dispersion given by the short time series is not as large. Therefore we conclude that the variance it is well estimated while the correlation time poorly estimated, then the first hypothesis of section 4.5.1 is validated. 


\section{A.2. NUMERICAL VALIDATION FOR SHORT TIME SERIES}

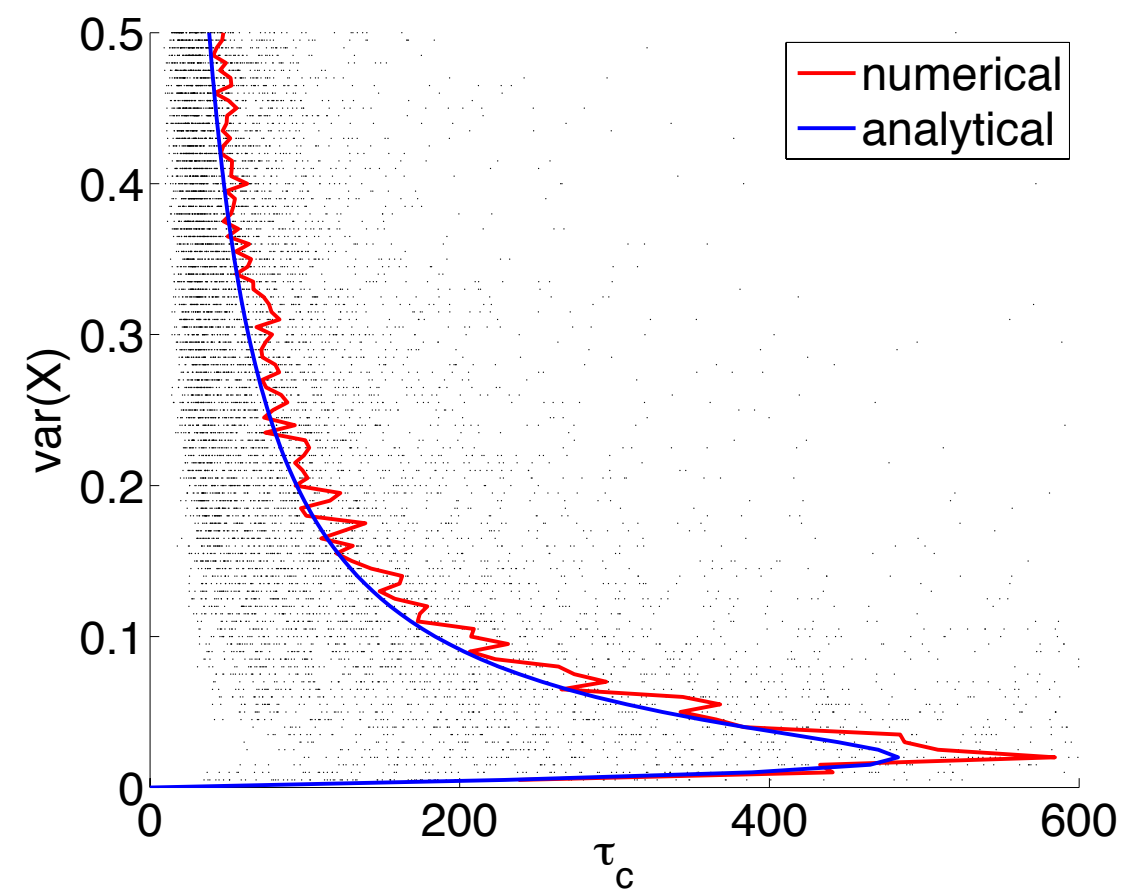

Figure A.1: Relation between $\tau_{c}$ and $\sigma^{2}$ obtained from numerical simulations of Equation 4.10 performed with the Euler-Maruyama method [47. The input parameters are parameters $D_{1}=0.2080, D_{2}=1.6 \times 10^{-4}$ and $\Delta t=0.01$, the values of $\sigma^{2}=k \Delta \sigma^{2}$ were obtained varying $k$ from 0 to 100 using $\Delta \sigma^{2}=.005$. For each value of $\sigma^{2}, 100$ realizations were performed, we can observe the agreement between the average value of the obtained parameters with the analytical calculation. 


\section{A.2. NUMERICAL VALIDATION FOR SHORT TIME SERIES}
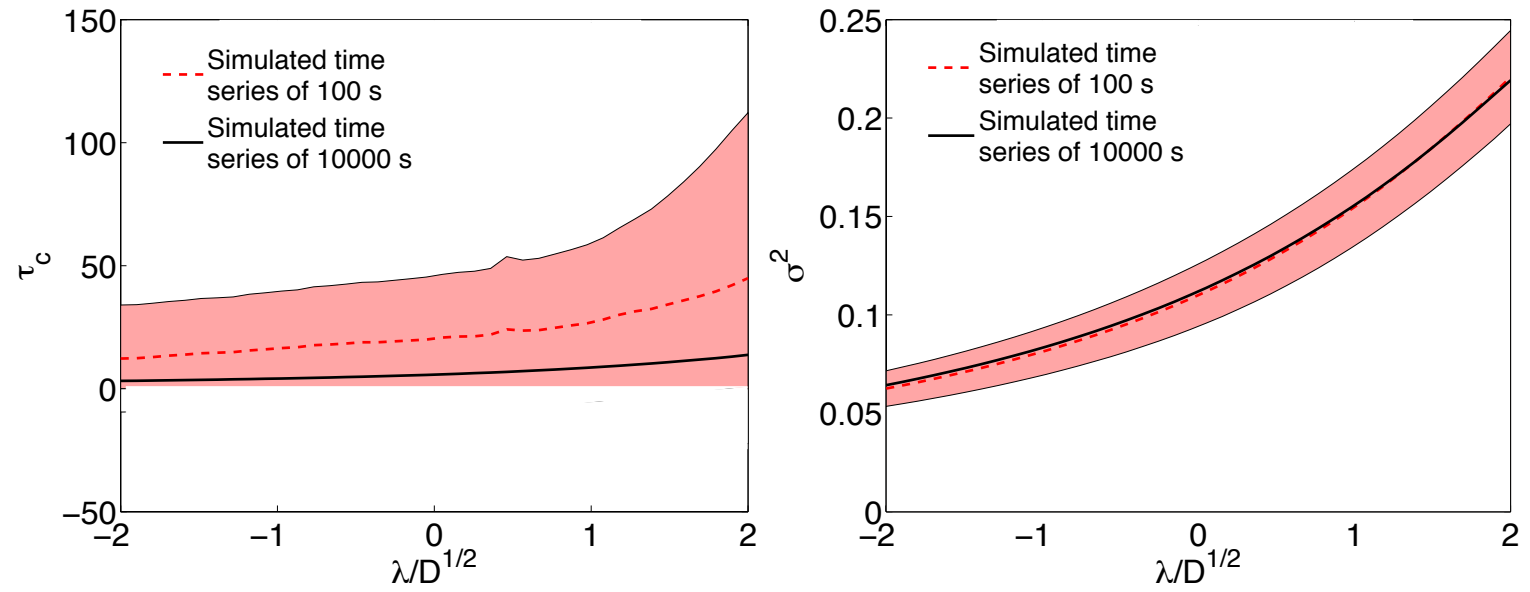

Figure A.2: Left, correlation time $\left(\tau_{c}\right)$ obtained by simulating time series of $100 \mathrm{~s}$ (red dashed line) and of $1000 \mathrm{~s}$ (black line). The colored area is denoting the variance obtained from the $100 \mathrm{~s}$. Right, variance $\left(\sigma^{2}\right)$ for short $(100 \mathrm{~s}$, red dashed line) and long time series (1000 s, black line), notice that both lines converge. 
A.2. NUMERICAL VALIDATION FOR SHORT TIME SERIES 


\section{Appendix B}

\section{Numerical simulations}

During this work we have performed several numerical simulations, using the EulerMaruyama algorithm [47], which is used for stochastic differential equations. In this Appendix we shall state the used parameters by figure. In our simulations we used always a random number generator from a commercial software package ( MATLAB R2012b, The MathWorks Inc., Natick, MA, 2012 ) that created numbers from a normal distribution with zero mean and variance of one.

\section{B.1 Figure 4.11 and A.1}

We performed several simulations of Equation 4.10, where we integrated

$$
\phi_{i+1}=\omega_{o} \Delta t+\left(\sqrt{\frac{D_{1}}{k \Delta \sigma^{2}}} \xi_{1 i}+\sqrt{D_{2} k \Delta \sigma^{2}} \xi_{2 i}\right) \sqrt{\Delta t}+\phi i
$$

with $\Delta t=0.01, D_{1}=0.2080, D_{2}=0.2080, \Delta \sigma^{2}=.005$ and varied $1 \leq k \leq 100$ for Figure A.1. For each value of $k$ used, 100 realizations were performed and the parameters correlation time, $\tau_{c}$, and variance, $\sigma^{2}$, were extracted from the autocorrelation function by doing a nonlinear fit as it was done in the experimental data. In Figure 4.11 a single realization was performed with the values $D_{1}=0.832$, $D_{2}=2 \times 10^{4}$ and $k \Delta \sigma=0.1423$. We used 10000 steps $\Delta t$ for each calculated time series. 


\section{B.2. FIGURE A.2}

\section{B.2 Figure A.2}

To test the artifacts given by estimating parameters from short time series, we simulated Equation 4.4

$$
\begin{aligned}
& x_{i+1}=\left(\lambda x_{i}-\omega_{o} y_{i}-\left(x_{i}^{2}+y_{i}\right)^{2}\right) \Delta t+\xi_{1 i} \sqrt{D \Delta t}+x_{i} \\
& y_{i+1}=\left(\lambda y_{i}+\omega_{o} x_{i}-\left(x_{i}^{2}+y_{i}\right)^{2}\right) \Delta t+\xi_{2 i} \sqrt{D \Delta t}+y_{i}
\end{aligned}
$$

with the parameters set to $\Delta t=0.01, D=0.1 \omega_{o}=1$. The values were varied $-0.2 \leq \lambda \leq 0.2$ with increments of $\Delta \lambda=0.004$, for the short time series of $100 \mathrm{~s}$ we simulated an ensemble of 100 realizations for each value of $\lambda$, while for the long time series of $10000 s$ a single realization was made.

\section{B.3 Figure 4.16}

The aim of this simulations was to show the mechanism for halting the self sustained oscillations. The model consists of Equations (4.16), 4.17) and 4.18). We simulated 10000 steps from the complete set of equations

$$
\begin{array}{r}
x_{i+1}=\left(\lambda_{i} x_{i}-\omega_{o} y_{i}-\left(x_{i}^{2}+y_{i}\right)^{2}-p_{i}\right) \Delta t+\xi_{1 i} \sqrt{D \Delta t}+x_{i} \\
y_{i+1}=\left(\lambda_{i} y_{i}+\omega_{o} x_{i}-\left(x_{i}^{2}+y_{i}\right)^{2}\right) \Delta t+\xi_{2 i} \sqrt{D \Delta t}+y_{i} \\
w_{i+1}=\left(-z_{i}-v_{i}+\frac{\beta}{2}\left(1-\lambda_{i}\right)\right) \Delta t+\xi_{3 i} \sqrt{D \Delta t}+w_{i} \\
v_{i+1}=\frac{1}{\tau}\left(w_{i}-v_{i}\right) \Delta t+\xi_{4 i} \sqrt{D \Delta t}+v_{i} \\
\lambda_{i}=-\tanh \left(\eta\left[w+\frac{w_{o}}{z_{o}} \sqrt{x^{2}+y^{2}}-w_{o}\right]\right)
\end{array}
$$

where the external perturbation was defined $p_{i}=7$ at $5000 \leq i \leq 5100$. The values were set to $\Delta t=0.01, \omega_{o}=5.0, D=.0150, \beta=1.5, \tau=33.33, \eta=10$, $w_{o}=1.0$ and $z_{o}=1.5$. The time series shown in Figure 4.15 (right) was taken from this simulation using $a_{i}=\sqrt{x_{i}^{2}+y_{i}^{2}}$. 


\section{B.4. FIGURE 4.15, LEFT}

\section{B.4 Figure 4.15, left}

We simulated the amplitude equation corresponding to Equations 4.13 and 4.15

$$
a_{i+1}=\left(-\lambda a_{i}+\epsilon a_{i}^{3}-\eta a_{i}^{5}+p_{i}\right) \Delta t+\xi_{i} \sqrt{D \Delta t}+a_{i}
$$

with the parameters $\lambda=1, \epsilon=2$ and $\eta=0.5$. The perturbation given was $p_{i}=-4.5$ at $5000 \leq i \leq 5100$, the number integration steps and $\Delta t$ are the same as in the previous section, as well as the value of $\sqrt{D}$.

\section{B.5 Figure 5.1 and 5.2}

To test our algorithm for data segregation, we have used a linear damped harmonic oscillator

$$
\begin{array}{r}
x_{i+1}=\left(-\lambda_{i} x_{i}-\omega_{o} y_{i}+p_{i}\right) \Delta t+\xi_{1 i} \sqrt{D \Delta t}+x_{i} \\
y_{i+1}=\left(-\lambda_{i} y_{i}+\omega_{o} x_{i}\right) \Delta t+\xi_{2 i} \sqrt{D \Delta t}+y_{i}
\end{array}
$$

where we have used $D=0.05, \Delta t=0.01$ and integrated for 7000 steps. The external perturbation was given at $1000 \leq i \leq 1900$ with a value of $p_{i}=-1$. In Figure 5.1 we have set the values of $\left\{\lambda, \omega_{o}\right\}$ as $\{0.1,0.4181\},\{0.15,0.523\}$, $\{0.20,0.402\}$ and $\{0.21,0.385\}$ for the curves of panels $1,2,3$ and 4 respectively.

To characterize the phase space distance $d_{i j}$ of Equation 5.9 we calculated a single reference trajectory $\{x, y\}$ with parameters $\lambda=0.1$ and $\omega_{o}=.3501$. As a next step we calculated a set of trajectories to measure their phase space distance with respect to the reference trajectory. We calculated these trajectories with different values of $\lambda=m \Delta \lambda$ and $\omega_{o}=n \Delta \omega_{o}$ with $\Delta \lambda=0.16$ and $\Delta \omega_{o}=0.0389$. We varied the values of $1 \leq m \leq 150$ and $1 \leq n \leq 150$ and the obtained trajectories were used to measure the phase space distance against the reference trajectory. 
B.5. FIGURE 5.1 AND 5.2 


\section{Appendix C}

\section{Samples of self sustained oscillations and colored noise}

In this section we present several measurements of cells that were classified as oscillatory in Chapter 3. In Figure C.1 we have six examples (out of seven) of cells that exhibited oscillations with high amplitude and in some cases with high correlation time. The data on these figures is raw with only its average for the first $100 s$ subtracted. In some cases the oscillations are detectable in both labels, indeed we have found that for many cells both signals are highly cross correlated. We conjecture that the dim signals do not reflect the actual expression of the protein. Naturally this question has been addressed before, where fluorescence levels have been found to be well correlated with the readings from western blots [119]. Though western blots represent a population average measure of protein expression and not from single cells. Also we have observed LimE responses from the experiments of Chapter 5, where the second label was not detectable, the response waveform was similar from the other cells. If we would assume that not detecting the second label represents the absence of the protein, then we would observed the similar response to one of a knockout, so far we have not observed that. Figure C.2 shows cases in which the signals have shown low correlation time. Some cases show very clearly that after perturbing the dynamics the activity of the cell continues, and in others cannot be discerned very well. Also it can be seen that in some cases both labels are very well cross correlated. 

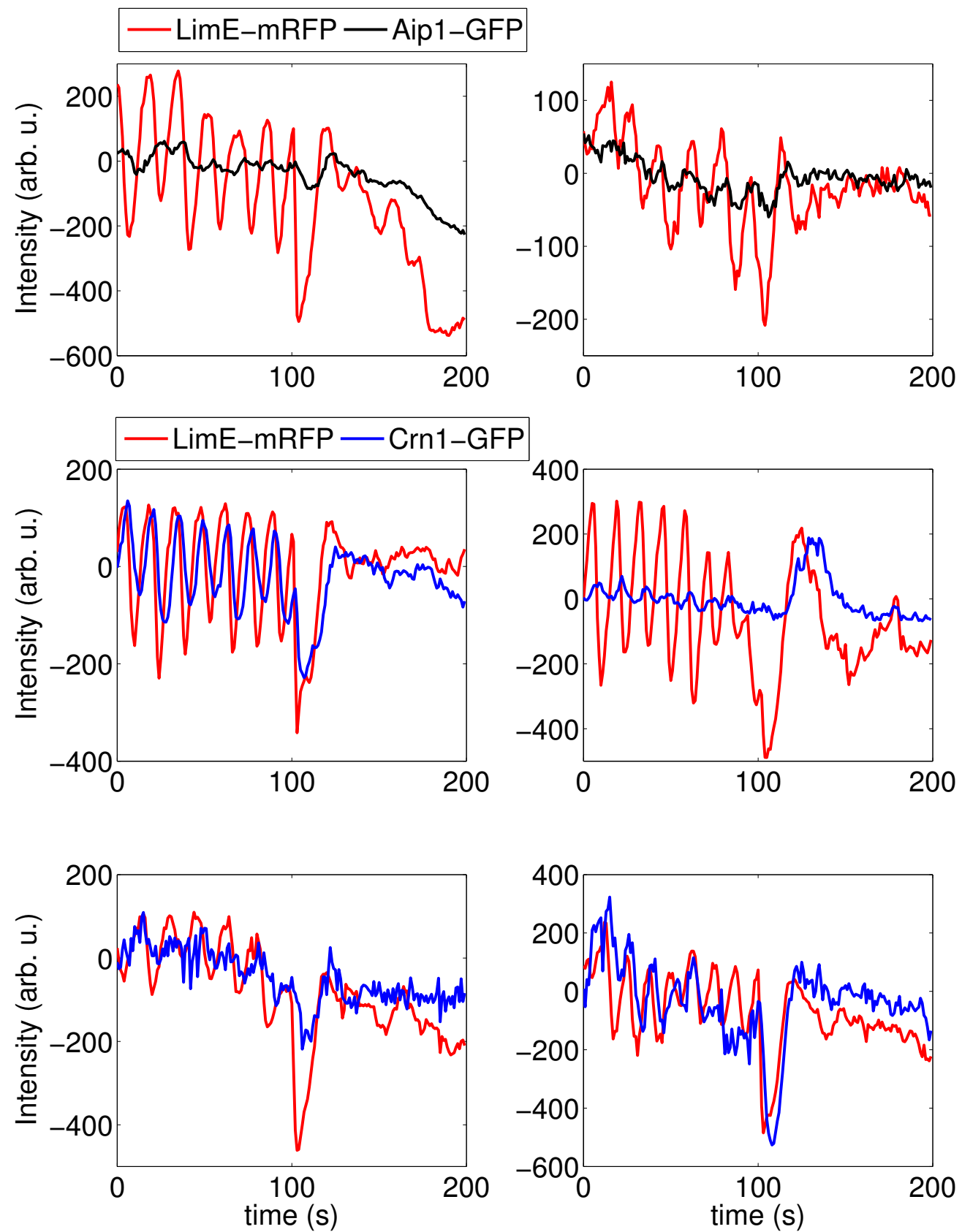

Figure C.1: Set of signals for LimE-mRFP+Aip1-GFP and LimE-mRFP+CrnGFP cells showing a long transient response after stimulation. This is a sample of the data set used in Chapter 3. 

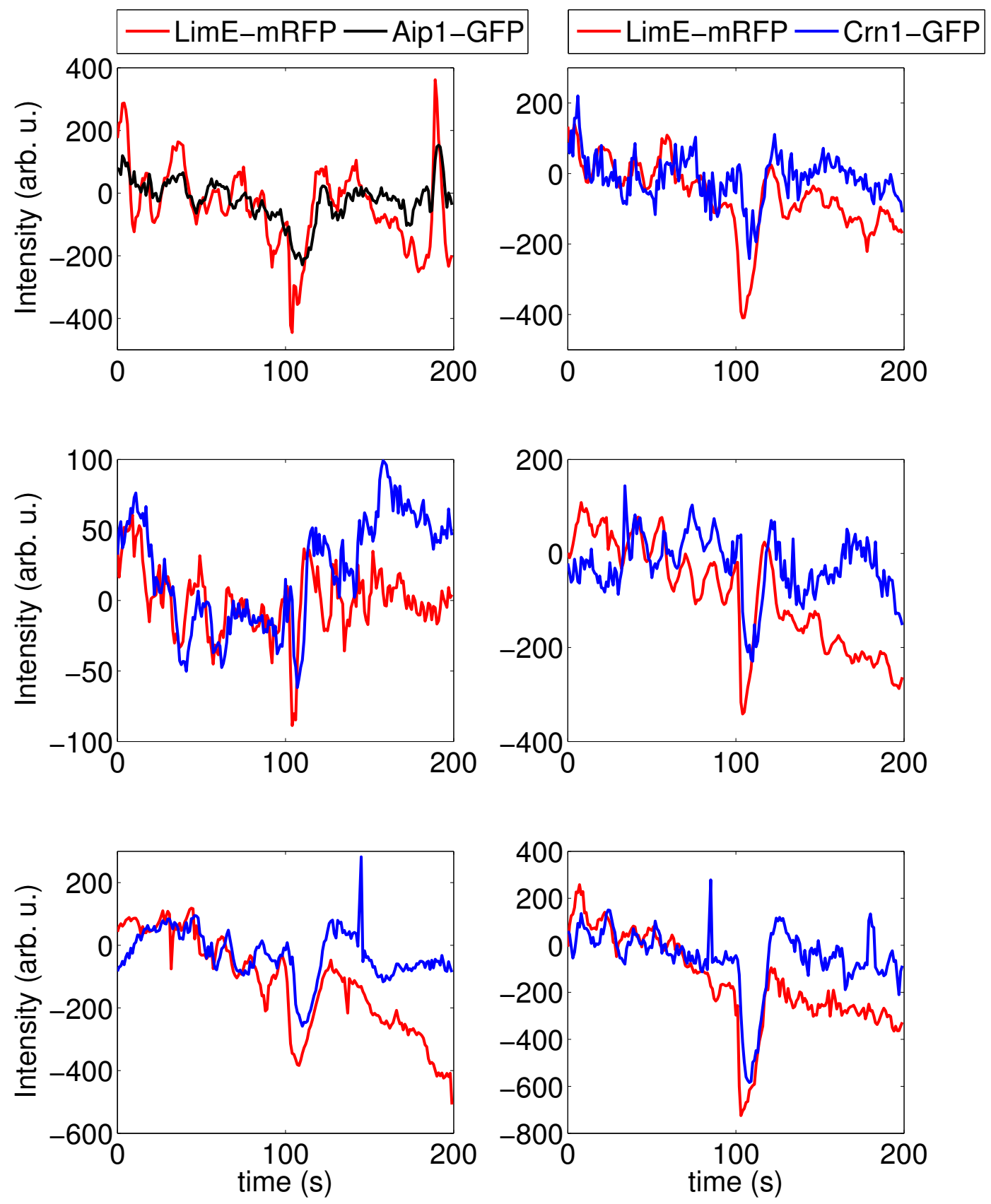

Figure C.2: Set of signals for LimE-mRFP+Aip1-GFP and LimE-mRFP+CrnGFP cells showing apparently a short transient response after stimulation. This is a sample of the data set used in Chapter 3. 


\section{Appendix D}

\section{Example of membrane retraction and oscillation}

When correlating fluorescence values with pseudopod extensions in Chapter 4, we found that the membrane can retract or oscillate. Retraction was define as a membrane front evolving towards the cytosol, and oscillation a membrane front that evolved outwards and then inwards.
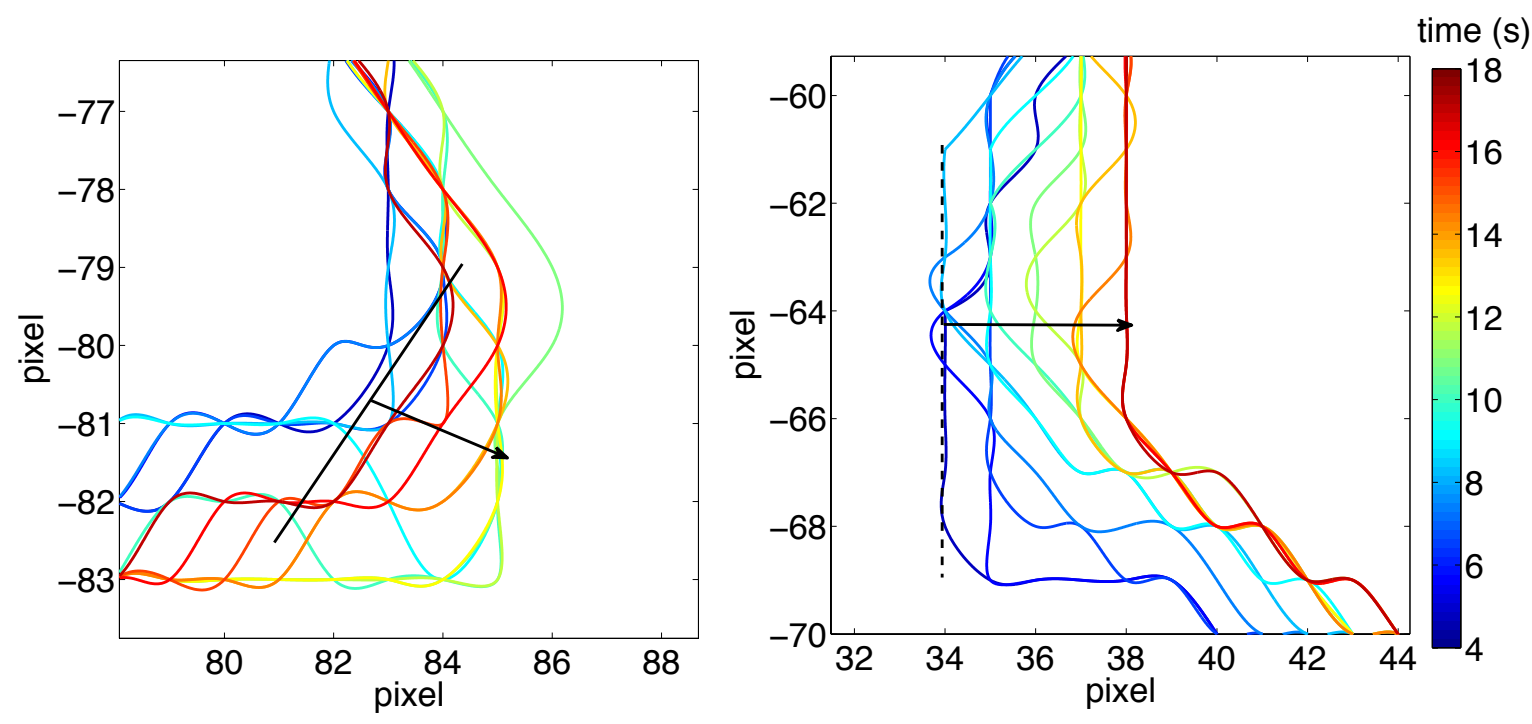

Figure D.1: Left, membrane oscillation and Right, membrane retraction. 


\section{Bibliography}

[1] B. Alberts, A. Johnson, J. Lewis, M. Raff, K. Roberts, and P. Walter. Molecular Biology of the Cell. Garland Science, 4th edition, 2002.

[2] U. Alon, M. G. Surette, N. Barkai, and S. Leibler. Robustness in bacterial chemotaxis. Nature, 397(6715):168-171, 011999.

[3] Gabriel Amselem, Matthias Theves, Albert Bae, Carsten Beta, and Eberhard Bodenschatz. Control parameter description of eukaryotic chemotaxis. Phys. Rev. Lett., 109:108103, Sep 2012.

[4] Yoshiyuki Arai, Tatsuo Shibata, Satomi Matsuoka, Masayuki J. Sato, Toshio Yanagida, and Masahiro Ueda. Self-organization of the phosphatidylinositol lipids signaling system for random cell migration. Proceedings of the National Academy of Sciences, 2010.

[5] R. Aris. On the dispersion of a solute in a fluid flowing through a tube. Proceedings of the Royal Society of London. Series A. Mathematical and Physical Sciences, 235(1200):67-77, 1956.

[6] Albert J. Bae, Carsten Beta, and Eberhard Bodenschatz. Rapid switching of chemical signals in microfluidic devices. Lab Chip, 9:3059-3065, 2009.

[7] Eric Batchelor, Alexander Loewer, and Galit Lahav. The ups and downs of p53: understanding protein dynamics in single cells. Nat Rev Cancer, 9(5):371-377, 052009.

[8] Carsten Beta, Danica Wyatt, Wouter-Jan Rappel, and Eberhard Bodenschatz. Flow photolysis for spatiotemporal stimulation of single cells. Analytical Chemistry, 79(10):3940-3944, 2007. 


\section{BIBLIOGRAPHY}

[9] Laurent Blanchoin, Kurt J. Amann, Henry N. Higgs, Jean-Baptiste Marchand, Donald A. Kaiser, and Thomas D. Pollard. Direct observation of dendritic actin filament networks nucleated by arp2/3 complex and wasp/scar proteins. Nature, 404(6781):1007-1011, 042000.

[10] Laurent Blanchoin, Rajaa Boujemaa-Paterski, Cécile Sykes, and Julie Plastino. Actin dynamics, architecture, and mechanics in cell motility. Physiological Reviews, 94(1):235-263, 2014.

[11] R.C. Bourret, U. Frisch, and A. Pouquet. Brownian motion of harmonic oscillator with stochastic frequency. Physica, 65(2):303 - 320, 1973.

[12] T Bretschneider, K Anderson, M Ecke, A Muller-Taubenberger, B SchrothDiez, HC Ishikawa-Ankerhold, and G Gerisch. The three-dimensional dynamics of actin waves, a model of cytoskeletal self-organization. Biophys $J$, 96(7):2888-2900, 2009.

[13] Till Bretschneider, Stefan Diez, Kurt Anderson, John Heuser, Margaret Clarke, Annette Müller-Taubenberger, Jana Köhler, and Günther Gerisch. Dynamic actin patterns and arp2/3 assembly at the substrate-attached surface of motile cells. Current Biology, 14(1):1 - 10, 2004.

[14] William M Brieher, Hao Yuan Kueh, Bryan A Ballif, and Timothy J Mitchison. Rapid actin monomer-insensitive depolymerization of listeria actin comet tails by cofilin, coronin, and aip1. J Cell Biol, 175(2):315-24, Oct 2006.

[15] Peter J. Brockwell and Richard A. Davis. Time Series: Theory and Methods. Springer New York, second edition, 2006.

[16] Sébastien Camalet, Thomas Duke, Frank Jülicher, and Jacques Prost. Auditory sensitivity provided by self-tuned critical oscillations of hair cells. Proceedings of the National Academy of Sciences, 97(7):3183-3188, 2000.

[17] Anders E. Carlsson. Actin dynamics: From nanoscale to microscale. Annual Review of Biophysics, 39(1):91-110, 2010. PMID: 20462375. 


\section{BIBLIOGRAPHY}

[18] Keefe T. Chan, Sarah J. Creed, and James E. Bear. Unraveling the enigma: progress towards understanding the coronin family of actin regulators. Trends in Cell Biology, 21(8):481-488, 2014/06/22 2011.

[19] T.F. Chan and L.A Vese. Active contours without edges. Image Processing, IEEE Transactions on, 10(2):266-277, Feb 2001.

[20] Michael G. Clark, Joseph Teply, Brian K. Haarer, Susan C. Viggiano, David Sept, and David C. Amberg. A genetic dissection of aip1p's interactions leads to a model for aip1p-cofilin cooperative activities. Molecular Biology of the Cell, 17(4):1971-1984, 2006.

[21] Patricia A. Clow and James G. McNally. In vivo observations of myosin ii dynamics support a role in rear retraction. Molecular Biology of the Cell, 10(5):1309-1323, 1999.

[22] M. Cross and H. Greenside. Pattern Formation and Dynamics in Nonequilibrium Systems. Cambridge University Press, 2009.

[23] E L de Hostos, B Bradtke, F Lottspeich, R Guggenheim, and G Gerisch. Coronin, an actin binding protein of dictyostelium discoideum localized to cell surface projections, has sequence similarities to g protein beta subunits. EMBO Journal, 10(13):4097-4101, 1991.

[24] E L de Hostos, C Rehfuess, B Bradtke, D R Waddell, R Albrecht, J Murphy, and G Gerisch. Dictyostelium mutants lacking the cytoskeletal protein coronin are defective in cytokinesis and cell motility. The Journal of Cell Biology, 120(1):163-173, 1993.

[25] Jaime de la Rocha, Brent Doiron, Eric Shea-Brown, Kresimir Josic, and Alex Reyes. Correlation between neural spike trains increases with firing rate. Nature, 448(7155):802-806, 082007.

[26] Juan C. del Álamo, Ruedi Meili, Baldomero Alonso-Latorre, Javier Rodríguez-Rodríguez, Alberto Aliseda, Richard A. Firtel, and Juan C. 


\section{BIBLIOGRAPHY}

Lasheras. Spatio-temporal analysis of eukaryotic cell motility by improved force cytometry. Proceedings of the National Academy of Sciences, 104(33):13343-13348, 2007.

[27] HG Dobereiner, BJ Dubin-Thaler, JM Hofman, HS Xenias, TN Sims, G Giannone, ML Dustin, CH Wiggins, and MP Sheetz. Lateral membrane waves constitute a universal dynamic pattern of motile cells. Phys Rev Lett, 97(3):038102, 2006.

[28] Mary J Dunlop, Robert Sidney Cox, Joseph H Levine, Richard M Murray, and Michael B Elowitz. Regulatory activity revealed by dynamic correlations in gene expression noise. Nat Genet, 40(12):1493-1498, 122008.

[29] David A. Egolf, Ilarion V. Melnikov, and Eberhard Bodenschatz. Importance of local pattern properties in spiral defect chaos. Phys. Rev. Lett., 80:32283231, Apr 1998.

[30] L. Eichinger, J. A. Pachebat, G. Glockner, M. A. Rajandream, R. Sucgang, M. Berriman, J. Song, R. Olsen, K. Szafranski, Q. Xu, B. Tunggal, S. Kummerfeld, M. Madera, B. A. Konfortov, F. Rivero, A. T. Bankier, R. Lehmann, N. Hamlin, R. Davies, P. Gaudet, P. Fey, K. Pilcher, G. Chen, D. Saunders, E. Sodergren, P. Davis, A. Kerhornou, X. Nie, N. Hall, C. Anjard, L. Hemphill, N. Bason, P. Farbrother, B. Desany, E. Just, T. Morio, R. Rost, C. Churcher, J. Cooper, S. Haydock, N. van Driessche, A. Cronin, I. Goodhead, D. Muzny, T. Mourier, A. Pain, M. Lu, D. Harper, R. Lindsay, H. Hauser, K. James, M. Quiles, M. Madan Babu, T. Saito, C. Buchrieser, A. Wardroper, M. Felder, M. Thangavelu, D. Johnson, A. Knights, H. Loulseged, K. Mungall, K. Oliver, C. Price, M. A. Quail, H. Urushihara, J. Hernandez, E. Rabbinowitsch, D. Steffen, M. Sanders, J. Ma, Y. Kohara, S. Sharp, M. Simmonds, S. Spiegler, A. Tivey, S. Sugano, B. White, D. Walker, J. Woodward, T. Winckler, Y. Tanaka, G. Shaulsky, M. Schleicher, G. Weinstock, A. Rosenthal, E. C. Cox, R. L. Chisholm, R. Gibbs, W. F. Loomis, M. Platzer, R. R. Kay, J. Williams, P. H. Dear, A. A. Noegel, B. Barrell, and A. Kuspa. The genome of the social amoeba dictyostelium discoideum. Nature, 435(7038):43-57, 052005. 


\section{BIBLIOGRAPHY}

[31] Michael B. Elowitz, Arnold J. Levine, Eric D. Siggia, and Peter S. Swain. Stochastic gene expression in a single cell. Science, 297(5584):1183-1186, 2002.

[32] Martin Etzrodt, Hellen C. F. Ishikawa, Jeremie Dalous, Annette MüllerTaubenberger, Till Bretschneider, and Günther Gerisch. Time-resolved responses to chemoattractant, characteristic of the front and tail of dictyostelium cells. FEBS letters, 580(28):6707-6713, 122006.

[33] Petra Fey, Anthony S Kowal, Pascale Gaudet, Karen E Pilcher, and Rex L Chisholm. Protocols for growth and development of dictyostelium discoideum. Nat. Protocols, 2(6):1307-1316, 062007.

[34] Markus Fischer, Ilka Haase, Evelyn Simmeth, Günther Gerisch, and Annette Müller-Taubenberger. A brilliant monomeric red fluorescent protein to visualize cytoskeleton dynamics in dictyostelium. FEBS Letters, 577(1):227-232, 2014/08/01 2004.

[35] Luca Gammaitoni, Peter Hänggi, Peter Jung, and Fabio Marchesoni. Stochastic resonance. Rev. Mod. Phys., 70:223-287, Jan 1998.

[36] Meghal Gandhi, Vérane Achard, Laurent Blanchoin, and Bruce L Goode. Coronin switches roles in actin disassembly depending on the nucleotide state of actin. Mol Cell, 34(3):364-74, May 2009.

[37] G. Gerisch and U. Wick. Intracellular oscillations and release of cyclic \{AMP\} from dictyostelium cells. Biochemical and Biophysical Research Communications, 65(1):364 - 370, 1975.

[38] Günther Gerisch, Richard Albrecht, Christina Heizer, Steve Hodgkinson, and Markus Maniak. Chemoattractant-controlled accumulation of coronin at the leading edge of dictyostelium cells monitored using a green fluorescent protein-coronin fusion protein. Current Biology, 5(11):1280-1285, 2014/08/01 1995. 


\section{BIBLIOGRAPHY}

[39] Gunther Gerisch, Mary Ecke, Dirk Wischnewski, and Britta SchrothDiez. Different modes of state transitions determine pattern in the phosphatidylinositide-actin system. BMC Cell Biology, 12(1):42, 2011.

[40] Günther Gerisch, Britta Schroth-Diez, Annette Müller-Taubenberger, and Mary Ecke. Pip3 waves and pten dynamics in the emergence of cell polarity. Biophysical Journal, 103(6):1170-1178, 2014/07/20 2012.

[41] Naama Geva-Zatorsky, Erez Dekel, Eric Batchelor, Galit Lahav, and Uri Alon. Fourier analysis and systems identification of the p53 feedback loop. Proceedings of the National Academy of Sciences, 107(30):13550-13555, 2010 .

[42] Leon Glass and Stuart A. Kauffman. The logical analysis of continuous, non-linear biochemical control networks. Journal of Theoretical Biology, 39(1):103 - 129, 1973.

[43] Thomas Gregor, Koichi Fujimoto, Noritaka Masaki, and Satoshi Sawai. The onset of collective behavior in social amoebae. Science, 328(5981):1021-1025, 2010 .

[44] J. Guckenheimer and P. Holmes. Nonlinear Oscillations, Dynamical Systems, and Bifurcations of Vector Fields. Number v. 42 in Applied Mathematical Sciences. Springer, 1983.

[45] Peter Hanggi, Thomas J. Mroczkowski, Frank Moss, and P. V. E. McClintock. Bistability driven by colored noise: Theory and experiment. Phys. Rev. A, 32:695-698, Jul 1985.

[46] Inbal Hecht, David A. Kessler, and Herbert Levine. Transient localized patterns in noise-driven reaction-diffusion systems. Phys. Rev. Lett., 104:158301, Apr 2010.

[47] D. Higham. An algorithmic introduction to numerical simulation of stochastic differential equations. SIAM Review, 43(3):525-546, 2001. 


\section{BIBLIOGRAPHY}

[48] Chuan-Hsiang Huang, Ming Tang, Changji Shi, Pablo A. Iglesias, and Peter N. Devreotes. An excitable signal integrator couples to an idling cytoskeletal oscillator to drive cell migration. Nat Cell Biol, 15(11):1307-1316, 112013.

[49] Miho Iijima and Peter Devreotes. Tumor suppressor pten mediates sensing of chemoattractant gradients. Cell, 109(5):599-610, 2014/07/07 2002.

[50] Hellen C. Ishikawa-Ankerhold, Günther Gerisch, and Annette MüllerTaubenberger. Genetic evidence for concerted control of actin dynamics in cytokinesis, endocytic traffic, and cell motility by coronin and aip1. $C y$ toskeleton, 67(7):442-455, 2010.

[51] Hellen Cristina Ishikawa-Ankerhold. Functional Organization in the Actin System of Dictyostelium. PhD thesis, Technischen Universität München, 2008.

[52] A. Isidori. Nonlinear Control Systems. Number v. 1 in Communications and Control Engineering. Springer, 1995.

[53] Chris Janetopoulos, Lan Ma, Peter N. Devreotes, and Pablo A. Iglesias. Chemoattractant-induced phosphatidylinositol 3,4,5-trisphosphate accumulation is spatially amplified and adapts, independent of the actin cytoskeleton. Proceedings of the National Academy of Sciences of the United States of America, 101(24):8951-8956, 2004.

[54] R.H. Kessin. Dictyostelium: Evolution, Cell Biology, and the Development of Multicellularity. Developmental and cell biology series. Cambridge University Press, 2001.

[55] Fabian Knoch, Marco Tarantola, Eberhard Bodenschatz, and Wouter-Jan Rappel. Modeling self-organized spatio-temporal patterns of pip 3 and pten during spontaneous cell polarization. Physical Biology, 11(4):046002, 2014.

[56] Angelika Konzok, Igor Weber, Evelyn Simmeth, Ulrike Hacker, Markus Maniak, and Annette Müller-Taubenberger. Daip1, a dictyostelium homologue 


\section{BIBLIOGRAPHY}

of the yeast actin-interacting protein 1, is involved in endocytosis, cytokinesis, and motility. The Journal of Cell Biology, 146(2):453-464, 1999.

[57] Arjan Kortholt, Ineke Keizer-Gunnink, Rama Kataria, and Peter J. M. Van Haastert. Ras activation and symmetry breaking during dictyostelium chemotaxis. Journal of Cell Science, 126(19):4502-4513, 2013.

[58] R. Kubo and N. Hashitsume. Statistical Physics II: Nonequilibrium Statistical Mechanics. Springer Series in Solid-State Sciences. Springer, 2012.

[59] Hao Yuan Kueh, Guillaume T Charras, Timothy J Mitchison, and William M Brieher. Actin disassembly by cofilin, coronin, and aip1 occurs in bursts and is inhibited by barbed-end cappers. J Cell Biol, 182(2):341-53, Jul 2008.

[60] Melvin Lax. Classical noise. v. noise in self-sustained oscillators. Phys. Rev., 160:290-307, Aug 1967.

[61] Andre Levchenko and Pablo A. Iglesias. Models of eukaryotic gradient sensing: Application to chemotaxis of amoebae and neutrophils. Biophysical Journal, 82(1):50 - 63, 2002.

[62] Jennifer Lippincott-Schwartz, Nihal Altan-Bonnet, and George H Patterson. Photobleaching and photoactivation: following protein dynamics in living cells. Nature cell biology, Suppl:S7-14, September 2003.

[63] Su-Ling Liu, Karen M. Needham, Jordan R. May, and Brad J. Nolen. Mechanism of a concentration-dependent switch between activation and inhibition of arp2/3 complex by coronin. Journal of Biological Chemistry, 286(19):17039-17046, 2011.

[64] Yang-Yu Liu, Jean-Jacques Slotine, and Albert-Laszlo Barabasi. Controllability of complex networks. Nature, 473(7346):167-173, 052011.

[65] Yang-Yu Liu, Jean-Jacques Slotine, and Albert-László Barabási. Observability of complex systems. Proceedings of the National Academy of Sciences, 110(7):2460-2465, 2013. 


\section{BIBLIOGRAPHY}

[66] M. Machacek and G. Danuser. Morphodynamic profiling of protrusion phenotypes. Biophysical Journal, 90(4):1439-1452, 2014/06/22 2006.

[67] F. Marks, U. Klingmüller, and K. Müller-Decker. Cellular signal processing: an introduction to the molecular mechanisms of signal transduction. Garland Science, 2009.

[68] P Martin, AJ Hudspeth, and F Jülicher. Comparison of a hair bundle's spontaneous oscillations with its response to mechanical stimulation reveals the underlying active process. Proceedings of the National Academy of Sciences, 98(25):14380-14385, 2001.

[69] Ruedi Meili, Baldomero Alonso-Latorre, Juan C. del Álamo, Richard A. Firtel, and Juan C. Lasheras. Myosin ii is essential for the spatiotemporal organization of traction forces during cell motility. Molecular Biology of the Cell, 21(3):405-417, 2010.

[70] Alphée Michelot, Alexandre Grassart, Voytek Okreglak, Michael Costanzo, Charles Boone, and David G. Drubin. Actin filament elongation in arp2/3derived networks is controlled by three distinct mechanisms. Developmental Cell, 24(2):182-195, 2014/06/22 2013.

[71] Namiko Mitarai, Uri Alon, and Mogens H. Jensen. Entrainment of noiseinduced and limit cycle oscillators under weak noise. Chaos: An Interdisciplinary Journal of Nonlinear Science, 23(2), 2013.

[72] Stephen W. Morris, Eberhard Bodenschatz, David S. Cannell, and Guenter Ahlers. Spiral defect chaos in large aspect ratio rayleigh-bénard convection. Phys. Rev. Lett., 71:2026-2029, Sep 1993.

[73] R. Dyche Mullins, John A. Heuser, and Thomas D. Pollard. The interaction of arp2/3 complex with actin: Nucleation, high affinity pointed end capping, and formation of branching networks of filaments. Proceedings of the National Academy of Sciences, 95(11):6181-6186, 1998.

[74] J.D. Murray. Mathematical Biology: I. An Introduction. Interdisciplinary Applied Mathematics. Springer, 2002. 


\section{BIBLIOGRAPHY}

[75] Masatoshi Nishikawa, Marcel Hörning, Masahiro Ueda, and Tatsuo Shibata. Excitable signal transduction induces both spontaneous and directional cell asymmetries in the phosphatidylinositol lipid signaling system for eukaryotic chemotaxis. Biophysical Journal, 106(3):723 - 734, 2014.

[76] A.A. Noegel and M. Schleicher. The actin cytoskeleton of dictyostelium: a story told by mutants. Journal of Cell Science, 113(5):759-766, 2000.

[77] Voytek Okreglak and David G. Drubin. Loss of aip1 reveals a role in maintaining the actin monomer pool and an in vivo oligomer assembly pathway. The Journal of Cell Biology, 188(6):769-777, 2010.

[78] Shoichiro Ono. Regulation of actin filament dynamics by actin depolymerizing factor/cofilin and actin-interacting protein 1: new blades for twisted filaments. Biochemistry, 42(46):13363-13370, 2003. PMID: 14621980.

[79] Juan M. Pedraza and Alexander van Oudenaarden. Noise propagation in gene networks. Science, 307(5717):1965-1969, 2005.

[80] Giuseppe Pesce, Austin McDaniel, Scott Hottovy, Jan Wehr, and Giovanni Volpe. Stratonovich-to-itôtransition in noisy systems with multiplicative feedback. Nat Commun, 4, 112013.

[81] Simone Pigolotti, Sandeep Krishna, and Mogens H. Jensen. Oscillation patterns in negative feedback loops. Proceedings of the National Academy of Sciences, 104(16):6533-6537, 2007.

[82] Simone Pigolotti, Sandeep Krishna, and Mogens H. Jensen. Symbolic dynamics of biological feedback networks. Phys. Rev. Lett., 102:088701, Feb 2009 .

[83] A. Pikovsky, M. Rosenblum, and J. Kurths. Synchronization: A Universal Concept in Nonlinear Sciences. Cambridge Nonlinear Science Series. Cambridge University Press, 2003.

[84] T D Pollard. Rate constants for the reactions of atp- and adp-actin with the ends of actin filaments. The Journal of Cell Biology, 103(6):2747-2754, 1986 . 


\section{BIBLIOGRAPHY}

[85] Thomas D Pollard and Gary G Borisy. Cellular motility driven by assembly and disassembly of actin filaments. Cell, 112(4):453-465, 2003.

[86] Marten Postma, Jeroen Roelofs, Joachim Goedhart, Theodorus W.J. Gadella, Antonie J.W.G. Visser, and Peter J.M. Van Haastert. Uniform camp stimulation of dictyostelium cells induces localized patches of signal transduction and pseudopodia. Molecular Biology of the Cell, 14(12):50195027, 2003.

[87] I. PRIGOGINE, R. LEFEVER, A. GOLDBETER, and M. HERSCHKOWITZ-KAUFMAN. Symmetry breaking instabilities in biological systems. Nature, 223(5209):913-916, 081969.

[88] Ilya Prigogine. Time, structure, and fluctuations. Science, 201(4358):777$785,1978$.

[89] Anne J. Ridley, Martin A. Schwartz, Keith Burridge, Richard A. Firtel, Mark H. Ginsberg, Gary Borisy, J. Thomas Parsons, and Alan Rick Horwitz. Cell migration: Integrating signals from front to back. Science, 302(5651):1704-1709, 2003.

[90] Julia Riedl, Alvaro H Crevenna, Kai Kessenbrock, Jerry Haochen Yu, Dorothee Neukirchen, Michal Bista, Frank Bradke, Dieter Jenne, Tad A Holak, Zena Werb, Michael Sixt, and Roland Wedlich-Soldner. Lifeact: a versatile marker to visualize f-actin. Nat Meth, 5(7):605-607, 072008.

[91] H. Risken. The Fokker Planck Equation: Methods of Solutions and Applications. Springer Series in Synergetics. Springer, 1996.

[92] Azadeh Samadani, Jerome Mettetal, and Alexander van Oudenaarden. Cellular asymmetry and individuality in directional sensing. Proceedings of the National Academy of Sciences, 103(31):11549-11554, 2006.

[93] Atsuo T. Sasaki, Cheryl Chun, Kosuke Takeda, and Richard A. Firtel. Localized ras signaling at the leading edge regulates pi3k, cell polarity, and directional cell movement. The Journal of Cell Biology, 167(3):505-518, 2004 . 


\section{BIBLIOGRAPHY}

[94] Satoshi Sawai, Peter A. Thomason, and Edward C. Cox. An autoregulatory circuit for long-range self-organization in dictyostelium cell populations. $\mathrm{Na}$ ture, 433(7023):323-326, 012005.

[95] Natalie Schneider, Igor Weber, Jan Faix, Josef Prassler, Annette MüllerTaubenberger, Jana Köhler, Emmanuel Burghardt, Günther Gerisch, and Gerard Marriott. A lim protein involved in the progression of cytokinesis and regulation of the mitotic spindle. Cell Motility and the Cytoskeleton, 56(2):130-139, 2003.

[96] Maria C Shina, Annette Müller-Taubenberger, Can Unal, Michael Schleicher, Michael Steinert, Ludwig Eichinger, Rolf Müller, Rosemarie Blau-Wasser, Gernot Glöckner, and Angelika A Noegel. Redundant and unique roles of coronin proteins in dictyostelium. Cell Mol Life Sci, 68(2):303-13, 2011.

[97] R.L. Stratonovich and R.A. Silverman. Topics in the Theory of Random Noise. Number v. 1 in Mathematics and its applications. Gordon and Breach, 1963.

[98] S.H. Strogatz. Nonlinear Dynamics and Chaos: With Applications to Physics, Biology, Chemistry, and Engineering. Addison-Wesley studies in nonlinearity. Westview Press, 1994.

[99] Karthic Swaminathan, Annette Müller-Taubenberger, Jan Faix, Francisco Rivero, and Angelika A Noegel. A cdc42- and rac-interactive binding (crib) domain mediates functions of coronin. Proc Natl Acad Sci USA, 111(1):E25-33, Jan 2014.

[100] Kristen F. Swaney, Chuan-Hsiang Huang, and Peter N. Devreotes. Eukaryotic chemotaxis: A network of signaling pathways controls motility, directional sensing, and polarity. Annual Review of Biophysics, 39(1):265-289, 2010. PMID: 20192768.

[101] Kosuke Takeda, Danying Shao, Micha Adler, Pascale G. Charest, William F. Loomis, Herbert Levine, Alex Groisman, Wouter-Jan Rappel, and Richard A. Firtel. Incoherent feedforward control governs adaptation 


\section{BIBLIOGRAPHY}

of activated ras in a eukaryotic chemotaxis pathway. Science Signaling, 5(205):ra2-ra2, 2012.

[102] Daisuke Taniguchi, Shuji Ishihara, Takehiko Oonuki, Mai Honda-Kitahara, Kunihiko Kaneko, and Satoshi Sawai. Phase geometries of two-dimensional excitable waves govern self-organized morphodynamics of amoeboid cells. Proceedings of the National Academy of Sciences, 2013.

[103] Jun-nosuke Teramae, Hiroya Nakao, and G. Bard Ermentrout. Stochastic phase reduction for a general class of noisy limit cycle oscillators. Phys. Rev. Lett., 102:194102, May 2009.

[104] L G Tilney and D A Portnoy. Actin filaments and the growth, movement, and spread of the intracellular bacterial parasite, listeria monocytogenes. The Journal of Cell Biology, 109(4):1597-1608, 1989.

[105] Roger Y. Tsien. The green fluorescent protein. Annual Review of Biochemistry, 67(1):509-544, 1998. PMID: 9759496.

[106] Masahiro Ueda, Yasushi Sako, Toshiki Tanaka, Peter Devreotes, and Toshio Yanagida. Single-molecule analysis of chemotactic signaling in dictyostelium cells. Science, 294(5543):864-867, 2001.

[107] N.G. Van Kampen. Stochastic Processes in Physics and Chemistry. NorthHolland Personal Library. Elsevier Science, 2011.

[108] Michael G. Vicker. Eukaryotic cell locomotion depends on the propagation of self-organized reaction-diffusion waves and oscillations of actin filament assembly. Experimental Cell Research, 275(1):54 - 66, 2002.

[109] Michael G Vicker. F-actin assembly in dictyostelium cell locomotion and shape oscillations propagates as a self-organized reaction-diffusion wave. FEBS Letters, 510(1):5-9, 2014/09/09 2002.

[110] Adrien F. Vinet, Thomas Fiedler, Vera Studer, Romain Froquet, Anna Dardel, Pierre Cosson, and Jean Pieters. Initiation of multicellular differentiation in dictyostelium discoideum is regulated by coronin a. Molecular Biology of the Cell, 25(5):688-701, 2014. 


\section{BIBLIOGRAPHY}

[111] C. Joanne Wang, Adriel Bergmann, Benjamin Lin, Kyuri Kim, and Andre Levchenko. Diverse sensitivity thresholds in dynamic signaling responses by social amoebae. Science Signaling, 5(213):ra17-ra17, 2012.

[112] D J Watts and J M Ashworth. Growth of myxameobae of the cellular slime mould dictyostelium discoideum in axenic culture. Biochem. J., 119(2):1710, 1970 .

[113] Christian Westendorf. Oscillatory Dynamics of the Actin Cytoskeleton. PhD thesis, Georg-August-Universität Göttingen, 2012.

[114] Christian Westendorf, Jose Negrete Jr, Albert J. Bae, Rabea Sandmann, Eberhard Bodenschatz, and Carsten Beta. Actin cytoskeleton of chemotactic amoebae operates close to the onset of oscillations. Proceedings of the National Academy of Sciences, 110(10):3853-3858, 2013.

[115] Monika Westphal, Andreas Jungbluth, Manfred Heidecker, Bettina Mühlbauer, Christina Heizer, Jean-Marc Schwartz, Gerard Marriott, and Günther Gerisch. Microfilament dynamics during cell movement and chemotaxis monitored using a gfp-actin fusion protein. Current Biology, 7(3):176$183,1997$.

[116] Kurt Wiesenfeld. Noisy precursors of nonlinear instabilities. Journal of Statistical Physics, 38(5-6):1071-1097, 1985.

[117] Kurt Wiesenfeld and Bruce McNamara. Small-signal amplification in bifurcating dynamical systems. Phys. Rev. A, 33:629-642, Jan 1986.

[118] Roy Wilds and Leon Glass. An atlas of robust, stable, high-dimensional limit cycles. International Journal of Bifurcation and Chaos, 19(12):4055-4096, 2009 .

[119] Jian-Qiu Wu and Thomas D. Pollard. Counting cytokinesis proteins globally and locally in fission yeast. Science, 310(5746):310-314, 2005.

[120] Min Wu, Xudong Wu, and Pietro De Camilli. Calcium oscillations-coupled conversion of actin travelling waves to standing oscillations. Proceedings of the National Academy of Sciences, 110(4):1339-1344, 2013. 


\section{BIBLIOGRAPHY}

[121] Yuan Xiong, Chuan-Hsiang Huang, Pablo A. Iglesias, and Peter N. Devreotes. Cells navigate with a local-excitation, global-inhibition-biased excitable network. Proceedings of the National Academy of Sciences, 107(40):17079-17086, 2010.

[122] Kazuyuki Yoshimura and Kenichi Arai. Phase reduction of stochastic limit cycle oscillators. Phys. Rev. Lett., 101:154101, Oct 2008.

[123] Sheng Zhang, Pascale G. Charest, and Richard A. Firtel. Spatiotemporal regulation of ras activity provides directional sensing. Current Biology, 18(20):1587-1593, 2008. 
BIBLIOGRAPHY 


\section{Acknowledgements}

This work could not have be done without the support and advice from so many people. First I would like to thank Prof. Dr. Eberhard Bodenschatz for letting me join his group in March of 2011, without his advices and constant challenges the quality of this work would have been much lower. Prof. Dr. Carsten Beta with whom I had plenty of discussions about data interpretation. This last year has been particularly pleasant since I have enjoyed working with Prof. Dr. Alain Pumir, many of the theoretical and experimental discoveries in Chapter 4 were the product of our discussions.

Also I have benefitted from the experimental training as well from discussion about biology from Dr. Christian Westendorf, whom I have inherited this project, and Dr. Marco Tarantola, whom as a group leader has made sure that my projects progress. The lab technicians, Katharina Schenider, Tina Althaus, Maren Stella Müller and Marion Kunze deserve a special mention since they always comply with my stringent demands for cell cultures.

Also I would like to thank the people who have dedicated part of their busy schedules to be part of my thesis and examination committees. Among them are Prof. Dr. Ulrich Parlitz and Prof. Dr. Sarah Köster who were members of my thesis committee, and as part of my examination committee Prof. Dr. Jörg Großhans, Dr. Andreas Neef and Reiner Kree.

In the life of a physicist it is very important to have the support of the people surrounding him. My wife Cristina G. Zubia Bueno and my daughters Andrea and Gala Isabel, which I love with all my heart, have been vital by encouraging and supporting my work and as well by making my daily life more enjoyable. My father José Negrete Almanza who was the person who encouraged analytical thinking since my early life and has supported my decision to become a physicist. 


\section{BIBLIOGRAPHY}

My mother Patricia Guerra Ortega who never stopped believing in me and has cared form me through all my life, and even though my departure abroad at age 23 has been a sad event in her life.

During my time in Göttingen I have benefitted from the friendship and support of several people among them: Fabio di Lorenzo, Christoph Blum, Regina Faubel, Noriko Oikawa, Azam Gholami, Vladimir Zykov, Kaumudi Prabhakara, Alexei Krekhov, Isabella Guido and Laura Turco. The list is even longer and please forgive me for people which I have not mentioned.

Finally I would like to thank the DFG SFB 937 - "Collective behavior of soft and biological matter" for financial support. 


\section{JOSÉ NEGRETE JR. \\ Curriculum Vitae}

PERSONAL INFORMATION

Nationality United States of America/ México

DOB $\quad 27$ January 1985

WORK EXPERIENCE

2011-2014 Max Planck institute for Dynamics and SelfOrganization - Göttingen, Germany

MPIDS Experimental and theoretical studies on the dynamics of the actin cytoskeleton and its regulators for Dictyostelium discoideum. Autonomous oscillations and fluctuations observed in the cytoskeleton were studied, along with physiological responses.

Supervisor: Prof. Dr. Eberhard Bodenschatz

2010 Laboratoire de Physique des Milieux Ionisés et Applications, Université de Nancy I - Nancy, France

LPMIA Master Student - Spatiotemporal analysis of plasma drift waves in the weakly turbulent regime and simulations of nonlinear dynamical systems.

Supervisor: Prof. Dr. Gérard Bonноммe

2007 National High Magnetic Field Laboratory, Los Alamos National Laboratory - NM, USA

NHMFL - LANL Intern - Experimental studies of magnetic frustration. Supervisor: Dr. Vivian ZAPF

\section{6-2008 The University of Texas at El Paso - TX, USA}

UTEP Research Assistant - Data analysis of collected airborne particulate matter for the environmental physics lab.

Supervisor: Prof. Dr. Rosa Fitzgerald

\section{EDUCATION}

\section{1-2014 Georg-August-Universität Göttingen, Germany}

Doctoral degree

Faculty of Physics

GGNB doctoral program Physics of Biological and Complex Systems Thesis: Fluctuations and oscillatory instabilities of intracellular fiber networks Advisor: Prof. Dr. Eberhard Bodenschatz

2008-2010 Erasmus Mundus Master In Nuclear Fusion and Engineering Physics, EU

Master of Science

Studies in plasma physics and nuclear fusion technology.

Studied at:

- Universidad Complutense de Madrid, Madrid, Spain (2008-2009)

- Küngliga Tekniska högskolan, Stockholm, Sweden (2009)

- Université de Nancy I - Henri Poincaré (currently known as Université de Lorraine) (2010)

Thesis: Characterization of Mode Locking and Energy Transfer in a Magnetized Plasma Column

Advisor: Prof. Dr. Gérard BonHomme

2003-2008 The University of Texas at El Paso, USA 
2000-2003 Escuela Preparatoria Federal por Cooperación

EMS-2/3 "El Chamizal”, Ciudad Juárez, México

Preparatory school

In preparation (advanced stage)

Phys Rev Lett

Proceedings of the National Academy of Sciences

Physical Review E

OTHER INFORMATION

Awards

Conferences

Languages

\section{RESEARCH ARTICLES} BODENSCHATZ and C. BETA and Biological Matter. Commission. Dead Sea, Israel. School, Geilo, Norway.
Basic high school education

2015 Fluctuations and transients in the oscillatory actin cytoskeleton of chemotactic amoeba.

J. Negrete Jr., A. Pumir, M. Tarantola, C. Westendorf, C. Beta and E.

2015 Spatiotemporal splitting of global eigenmodes due to cross-field coupling via vortex dynamics in drift wave turbulence.

C. Brandt, S.C. Thakur, A.D. Light, J. Negrete JR., and G.R. Tynan

2013 Actin cytoskeleton of chemotactic amoeba operates close to the onset of oscillations.

C. Westendorf, J. Negrete Jr., A.J. Bae, R. Sandmann, E. Bodenschatz

2011 Spatiotemporal mode structure of nonlinearly coupled drift wave modes

C. Brandt, O. Grulke, T. Klinger, J. Negrete Jr., G. Bousselin, F. Brochard, . BONHOMme, and S. Oldenbürger

2013 . EPL Poster Awards 2013 of the DPG Biological Physics Division (shared with Christian Westendorf).

2011 - PhD studentship grant from DFG SFB 937 Collective Behavior of Soft

2008 · Erasmus Mundus Master Scholarship awarded by the European

2007 - REU grant (Research Experience for Undergraduates) given by the National Science Foundation, USA

February 2014 · 18th Harz Seminar: Pattern Formation in Chemistry and Biophysics, Hahnenklee, Germany.

April 2013 - EMBO SPATIAL 2013: From Spatial Sensing to Sensing Spatiality,

March 2013 - Soft Matter Confinement: From Biology to Physics Winter

June 2012 • Biological Complex Fluids Summer School, Cargèse, France.

\footnotetext{
SPANISH $\cdot$ Native

ENGLISH · Full professional proficiency

JAPANESE · Elementary
} 